\title{
Energy scales and black hole pseudospectra: the structural role of the scalar product
}

\author{
E. Gasperín and J.L. Jaramillo \\ Institut de Mathématiques de Bourgogne (IMB), UMR 5584, CNRS, Université de \\ Bourgogne Franche-Comté, F-21000 Dijon, France.
}

\begin{abstract}
.
A pseudospectrum analysis has recently provided evidence of a potential generic instability of black hole $(\mathrm{BH})$ quasinormal mode $(\mathrm{QNM})$ overtones under high-frequency perturbations. Such instability analysis depends on the assessment of the size of perturbations. The latter is encoded in the scalar product and its choice is not unique. Here, we address the impact of the scalar product choice, advocating for founding it on the physical energy scales of the problem. The article is organized in three parts: basics, applications and heuristic proposals. In the first part, we revisit the energy scalar product used in the hyperboloidal approach to QNMs, extending previous effective analyses and placing them on solid spacetime basis. The second part focuses on systematic applications of the scalar product in the QNM problem: i) we demonstrate that the QNM instability is not an artifact of previous spectral numerical schemes, by implementing a finite elements calculation from a weak formulation; ii) using Keldysh's asymptotic expansion of the resolvent, we provide QNM resonant expansions for the gravitational waveform, with explicit expressions of the expansion coefficients; iii) we propose the notion of 'epsilon-dual QNM expansions' to exploit BH QNM instability in BH spectroscopy, complementarily exploiting both non-perturbed and perturbed QNMs, the former informing on large scales and the latter probing small scales. The third part enlarges the conceptual scope of BH QNM instability proposing: a) spiked perturbations are more efficient in triggering BH QNM instabilities than smooth ones, b) a general picture of the $\mathrm{BH}$ QNM instability problem is given, supporting the conjecture (built on Burnett's conjecture on the spacetime high-frequency limit) that Nollert-Price branches converge universally to logarithmic Regge branches in the high-frequency limit and c) aiming at a fully geometric description of QNMs, BMS states are hinted as possible asymptotic/boundary degrees of freedom for an inverse scattering problem.
\end{abstract}

\section{Contents}

1 Introduction: scales and QNM instability

2 Hyperboloidal approach to QNMs: the energy scalar product in a nutshell 4

2.1 Energy scalar product: basics . . . . . . . . . . . . 5

2.1.1 Assessing big and small in spectral stability problems: the relevance of the norm. . . . . . . . . . . . . . . . . 5

2.1.2 Physical scales: energy norm and scalar product in the hyperboloidal

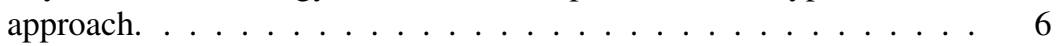

2.2 Energy scalar product: applications . . . . . . . . . . . . 7

2.2.1 QNM weak formulation and finite element calculations. . . . . . 7

2.2.2 QNM resonant expansions: a Keldysh's projection expression $\ldots . .7$

2.3 Energy scalar product: general picture, heuristics and geometry. . . . . . 8

2.3.1 Building a general picture of QNM ultraviolet instability. . . . . . . 8 
2.3.2 BH spectroscopy: $\epsilon$-dual QNM expansions. . . . . . . . . . . 8

2.3.3 Operator norm and metric fluctuations. . . . . . . . . . . 9

2.3.4 QNM instability and compact object QNM universality: from highfrequency Burnett's conjecture to a Regge-QNM's conjecture. . . . . 10

2.3.5 Towards a geometric formulation of QNM instability. . . . . . . . . . 10

3 QNM spectral instability, pseudospectrum and scalar product choice 11

3.1 Spectral instability as a norm-dependent notion: a study case . . . . . . . . . 11

3.1.1 Scalar product, selfadjointness and normality. . . . . . . . . . . 12

3.1.2 QNM stability through the pseudospectrum: the role of the scalar product. ...................... 14

3.2 Hyperboloidal slicing framework: the energy scalar product choice . . . . . . 17

3.2.1 Elements of the hyperboloidal framework in spherical symmetry. . . 17

3.2.2 Total energy versus effective mode energy. . . . . . . . . . . . . 20

3.2.3 Energy flux at spacetime boundaries. . . . . . . . . . . 22

4 Scalar product in the hyperboloidal approach to QNMs: some applications 24

4.1 Energy scalar product and QNM weak formulation . . . . . . . . . . . 24

4.1.1 Weak formulation of the QNM problem. . . . . . . . . . . 25

4.1.2 Formal adjoint $L^{\dagger}$ in the weak formulation. . . . . . . . . . 26

4.1.3 QNM calculation in the weak formulation: finite elements. . . . . . . 26

4.2 Scalar product and QNM resonant expansions: towards BH spectroscopy . . 28

4.2.1 Resolvent of non-selfadjoint operators: Keldysh's expansion. . . . . . 29

4.2.2 QNM resonant expansions in the hyperboloidal approach. . . . . . 30

4.2.3 Keldysh QNM expansion for the scattered field. . . . . . . . . . 33

5 QNM spectral instability: an emerging general picture 35

5.1 Elements in the QNM spectral instability problem . . . . . . . . . . 35

5.1.1 QNM-free regions: logarithmic boundaries and pseudospectra. . . . 35

5.1.2 Regular high-frequency QNM perturbations: Nollert-Price-like

branches and "inner" QNMs. . . . . . . . . . . . . . . 36

5.1.3 Non-regular $C^{p}$ QNM perturbations: logarithm Regge branches. . . . 38

5.2 Emerging picture: universality in QNMs of compact objects . . . . . . . . 40

5.3 BH spectroscopy and $\epsilon$-dual QNM resonant expansions. . . . . . . . . . . . 41 4

5.3.1 GW signal time domain stability versus QNM spectral instability. . . 41

5.3.2 Data analysis inverse problem: perturbation-enhanced degeneracy. . . 43

6 Geometry and QNM instability: heuristics on further structural questions 43

6.1 Physical interpretation of the energy norm: role of spacetime density perturbations ....................... 43

6.2 High-frequency limit of general relativity and spacetime regularity: Burnett's conjecture and QNM high-frequency perturbations . . . . . . . . . . . 48

6.2.1 Ultraviolet QNM perturbations: Regge QNM branches conjecture. . . 49

6.3 Towards a geometric description of QNMs . . . . . . . . . . . . . 50

6.3.1 Compactified hyperboloidal slicing: geometric data. . . . . . . . . 51

6.3.2 Geometric formulation of dynamics and scalar product. . . . . . . . . 51

6.3.3 A geometric approach to QNM characterization. . . . . . . . 52 52

6.3.4 Missing degrees of freedom and BMS symmetry: towards normal modes in isolated objects. . . . . . . . . . . . . . 53 
7 Conclusions and perspectives

7.1 Conclusions . . . . . . . . . . . . . . . . . . . 54

7.1.1 Complementary developments in the QNM instability problem. . . . 56

7.2 Perspectives .................... 56

PACS numbers:

\section{Introduction: scales and QNM instability}

The goal of the present article is to address some conceptual and technical issues concerning the role of the scalar product in the analysis of BH QNM instability, namely in the setting of a recently introduced pseudospectrum framework [53]. Spectral instability of QNM overtones under high-frequency perturbations is a phenomenon of potential relevance in the study of basic properties of BHs, in particular probing their small scale physics. Specifically, it may be of interest for fundamental gravity at the quantum scale and, given current and future prospects regarding interferometric antennae, for gravitational wave (GW) physics and the emerging field of BH spectroscopy [11, 27, 6, 82, 49, 38, 48, 18, 83].

QNMs play a central role in the modeling of (scattering) dissipative systems with applications in research areas so diverse as spectroscopy, optics, oceanography or general relativity, to cite some examples. In the latter gravitational case, QNMs appear naturally in the study of linear perturbations of BHs and in the study of propagating fields on black hole backgrounds - see [61, 80, 10, 62] for comprehensive reviews (in particular also addressing QNMs of other classes of compact objects, e.g. neutron stars). The dissipative nature of scattering by black holes follows from the presence of the event horizon and null infinity. In the case of, for example, optics, QNMs appear in the study of the interaction of light and plasmonic nanoresonators [91, 65] and in the study of optical leaky cavities [70, 69, 19, 16].

Regardless of the specific physical setup in consideration, the dissipative behavior is encoded, at the mathematical level, in the lack of selfadjointness of the operator governing the dynamics of perturbations to the physical system. In the scattering setting, this follows in particular from the imposed outgoing boundary conditions. QNMs in all these contexts can be shown to correspond to the eigenvalues of such non-selfadjoint operators. This statement can be made precise, in particular, by using a hyperboloidal formulation of the equations [110, 3, 41, 53]. The non-selfadjoint nature of the operator makes these eigenvalues complex and alter profoundly the properties of the set of eigenfunctions, in particular their completeness and orthogonality. Hence the prefix quasi is used to distinguish them from the usual normal modes of selfadjoint operators which have real eigenvalues with eigenfunctions forming orthonormal bases. Thus, in such a setting, the study of QNMs can be thought condensedly as an application of the spectral analysis theory for non-selfadjoint operators.

A natural question that arises is the robustness —or structural stability- of QNMs in such physical systems, namely, how much the QNM spectrum changes upon (small) perturbations of the operator. In other words, an assessment of the spectral QNM (in)stability. In the case of normal operators (in particular selfadjoint ones) spectral stability is ensured by the spectral theorem. In contrast, non-normal operators can potentially be spectrally unstable. A systematic tool to investigate the spectral instability of a given operator is the analysis of its pseudospectrum - see [64, 25, 96] for precise definitions and an exhaustive discussion. Heuristically, the pseudospectrum of an operator can be thought as a cartographic map of its analytic structure (actually, of its resolvent) providing, in particular, a tool that can be used to qualitatively read off "the sensitivity" of an operator upon perturbations. The analysis of the pseudospectrum has been exploited in several areas of physics where eigenvalues of non- 
selfadjoint operators arise such as hydrodynamics and turbulence or non-Hermitian quantum mechanics - see [103, 63], and has been recently introduced in gravitational physics in [53] in the context of BH QNMs (cf. [30] for extensive discussion of pseudospectrum applications).

One of the equivalent characterizations of the pseudospectrum, which is suitable in the present setting, is that the pseudospectrum of an operator $L$ consists of the set of points in the complex plane that are realized as eigenvalues of some perturbation $L+\delta L$ of the original operator $L$. To address possible spectral instabilities, one is interested in estimating how large the change in the eigenvalues, produced by small perturbations of the operator, can be. Such variation range defines the so-called $\epsilon$-pseudospectra sets associated with an $\epsilon$-sized operator perturbations. We will say that the system is spectrally stable if, under operator perturbations of 'size' $\epsilon$, the change in the eigenvalues is also of order $\epsilon$. On the contrary, if small changes of order $\epsilon$ in the operator give rise to variations in the spectrum (orders-of-magnitude) much larger than $\epsilon$, then we say that $L$ is spectrally unstable. Therefore the assessment of spectral instability critically depends on the "rule" we choose to determine the notion of 'big' and 'small' in the size of operator perturbations, namely the employed operator norm. Thus, in contrast with the notion of spectrum which is an intrinsic property of the operator, the notion of pseudospectrum — and therefore the notion of (in)stability — depends also on the choice of norm. This is crucial, since perturbations considered as small in one norm could correspond to large perturbations in another in another norm. In sum, the appropriate choice of scalar product — with its associated norm — becomes a key aspect in the study of QNM (in)stability.

From a mathematical perspective, the study of the spectral stability of a non-normal operator can be linked to assessing the existence of a scalar product permitting to keep the control of eigenvalue perturbations in tiny sets around the spectrum, i.e with $\epsilon$-pseudospectra sets tighly packed around the spectrum. However, from a physical perspective, the notion of large and small can be actually fixed by the physics of the problem, therefore not having necessarily the freedom of making up a norm to mathematically "control" the instabilities.

The analysis of this question defines the problem addressed in this work. In particular, we pursue further the program initiated in [53] and extended in [54] — with a first analysis on the implications on GW physics: assessment of the presence of perturbed QNMs in the GW signal, isospectrality loss and the introduction effective parameters for data analysis- and in [26] - with a first exploration of asymptotic universality and the geometric character of the pseudospectrum, namely the slicing independence inside the hyperboloidal approach. In a further step, in this article we discuss the role of the inner product and its associated norm for assessing the spectral (in)stability of QNMs and some of its implications. In the following, we summarize the main results to be developed later in the text.

\section{Hyperboloidal approach to QNMs: the energy scalar product in a nutshell}

The present work is framed in a compactified hyperboloidal approach to the scattering problem of a field $\Phi$ in stationary asymptotically flat black hole (BH) spacetimes (cf. [53, 54] for details; see also [3, 85, 84]). Specifically, we deal with linear wave equations in a firstorder reduction in time, with spatial slices $\Sigma_{\tau}$ intersecting the BH horizon and null infinity. As a methodological simplification, we focus on spherically symmetric spacetimes, leading to reduced $1+1$-dimensional problems for harmonic modes $\phi_{\ell m}$. Dropping the mode labels $(\ell m)$ and writing $\psi=\partial_{\tau} \phi$, the wave equation is written as

$$
\partial_{\tau} u=i L u, u=\left(\begin{array}{l}
\phi \\
\psi
\end{array}\right),
$$


with the operator $L$ given by

$$
L=\frac{1}{i}\left(\begin{array}{c|c}
0 & 1 \\
\hline L_{1} & L_{2}
\end{array}\right),
$$

For scalar field potentials we have (here $x$ denotes a coordinate parametrizing spheres in $\Sigma_{\tau}$ )

$$
L_{1}=\frac{1}{w(x)}\left(\partial_{x}\left(p(x) \partial_{x}\right)-\tilde{V}(x)\right), \quad L_{2}=\frac{1}{w(x)}\left(2 \gamma(x) \partial_{x}+\partial_{x} \gamma(x)\right)
$$

where functions $p(x)$ and $\tilde{V}(x)$ depend only on the intrinsic geometry of the slice $\Sigma_{\tau}$, whereas the function $\gamma(x)$ and the weight function $w(x)$ depend also on the extrinsic geometry of $\Sigma_{\tau}$ in the spacetime (see details in [53]).

A key point in the discussion concerns the choice of a scalar product, defining the Hilbert space in which solutions $u$ to equation (1) live and, crucially for our discussion, defining the adjoint operator $L^{\dagger}$. This article focuses on different questions around such scalar product choice. Given the broad range of discussed aspects, the rest of this section summarises the main points later developed in the text, with the aim of acquiring an overall picture of the involved problems and indicating the sections where corresponding results are presented.

\subsection{Energy scalar product: basics}

This subsection focuses on structural issues regarding the scalar product and its associated norm, namely its role in the construction of the pseudospectrum. Then, upon the specific choice of an "energy scalar product", we adress the relation between the total spacetime problem and the reduced spherically-symmetric problem that was studied in [53].

2.1.1. Assessing big and small in spectral stability problems: the relevance of the norm. Taking the Fourier transform in $\tau$ of equation (1), the QNM problem can be cast as an eigenvalue problem for a non-selfadjoint operator $L$

$$
L v_{n}=\omega_{n} v_{n} .
$$

If one considers, for $\epsilon>0$, a perturbation $\delta L(\epsilon)$ of norm $\epsilon$, the perturbed (right-)eigenvalue problem is written as

$$
(L+\delta L) v_{n}^{\epsilon}=\omega_{n}^{\epsilon} v_{n}^{\epsilon},\|\delta L\|=\epsilon .
$$

Then it holds (cf. e.g. [58, 105])

$$
\left|\omega_{n}^{\epsilon}-\omega_{n}\right| \leq \epsilon \kappa_{n}+O\left(\epsilon^{2}\right),
$$

where $\kappa_{n}$ is the so-called condition number of $\omega_{n}$ (see definition later in equation (128)). We say that $\omega_{n}$ is unstable if perturbations with small $\|\delta L\|=\epsilon$ produce large changes $\left|\omega_{n}^{\epsilon}-\omega_{n}\right|$, associated with large values of $\kappa_{n}$. Therefore, we need to control the notion of 'big' and 'small' perturbations, encoded in the operator norm $\|\cdot\|$ for $\delta L$, constructed from a vector norm $\|\cdot\|$ (we abuse the repeated notation here) associated with a scalar product $\langle\cdot, \cdot\rangle$.

A complementary approach to spectral instability is given by the notion of pseudospectrum [105, 25, 97]. Such notion captures how far perturbed eigenvalues can migrate in the complex plane under an operator perturbation with $\|\delta L\|=\epsilon$ (see [53] for a detailed discussion in the present context). Specifically, the notion of $\epsilon$-pseudospectrum set 
$\sigma^{\epsilon}(L)$ can be equivalently characterized as (here $\sigma(L)$ is the spectrum $\ddagger$ of $L$ )

$$
\begin{aligned}
\sigma^{\epsilon}(L) & =\left\{\lambda \in \mathbb{C}, \exists \delta L \in M_{n}(\mathbb{C}),\|\delta L\|<\epsilon: \lambda \in \sigma(L+\delta L)\right\} \\
& =\left\{\lambda \in \mathbb{C}:\left\|(\lambda \mathrm{Id}-L)^{-1}\right\|>1 / \epsilon\right\} \\
& =\left\{\lambda \in \mathbb{C}, \exists v \in \mathbb{C}^{n}:\|L v-\lambda v\|<\epsilon\right\} .
\end{aligned}
$$

As above in equations $(5)-(6)$, norms appear explicitly in the construction of the sets $\sigma^{\epsilon}(L)$, inside which eigenvalues can migrate under operator perturbations. Therefore, the control of notions of 'big' and 'small' is in-built in the notion of spectral stability.

In section 3.1 we discuss a simple example that dramatically illustrates the impact of the choice of scalar product and its norm in the assessment of spectral instability in nonselfadjoint operators. In particular, it neatly highlights the impact of the norm choice in the construction of the pseudospectrum.

2.1.2. Physical scales: energy norm and scalar product in the hyperboloidal approach. A key element in the pseudospectrum discussion in [53] is the use of a so-called "energy scalar product", encoding the geometric and analytical structure of the problem. In particular, in the assessment of big/small perturbations, this translates into an operator norm aiming at capturing the relevant scales in terms of the energy. Such "energy (operator) norm" (see also [28, 92, 104]) is induced from a vector norm defined directly from the energy of perturbations. Given a perturbation $\Phi$ with associated stress-energy tensor $T_{a b}$ (in a stationary spacetime with timelike Killing $t^{a}$ ), its energy $E(\Phi)[109]$ in a time slice $\Sigma_{\tau}$ defines the norm $\|\Phi\|_{E}$

$$
\|\Phi\|_{E}:=E(\Phi)=\int_{\Sigma_{\tau}} T_{a b}(\Phi) t^{a} n^{b} d \Sigma
$$

with $n^{a}$ the timelike normal to $\Sigma$. Two caveats are associated with the use of such a norm. First, the operator norm $\|\delta L\|_{E}$ of a perturbation $\delta L$ is induced from the vector energy norm (8), but it is not itself an energy. Therefore, the relation between the energy in field perturbations and the assessment of scales of QNM instabilities is a point needing elucidation, something that will be discussed in section 6.1. Second, and more technically, the energy norm constructed in [53] for spherically symmetric problems is not built on the full stress-energy tensor $T_{a b}$ of $\Phi$, but actually on the effective stress-energy tensor $\stackrel{\circ}{T}_{a b}$ associated with the $1+1$ dimensional problem for a given mode $\phi_{\ell m}$ (in a spherical harmonic decomposition). A key question is then: how does the energy $E_{\ell m}:=E\left(\phi_{\ell m}\right)$ relates to the total energy $E(\Phi)$ ? Section 3.2 answers this question for a scalar field, giving the expected (but significant) result

$$
E=\sum_{\ell m}{\stackrel{\circ}{E_{\ell m}}}-\left.\left(\frac{f}{2 r} \phi_{\ell m} \bar{\phi}_{\ell m}\right)\right|_{a} ^{b},
$$

where $a$ and $b$ represent the (compactified) boundaries in our problem, $f$ is the function in the line element in Schwarzschild coordinates vanishing at the horizon and $r$ is the (Schwarzschild) radial coordinate (diverging at infinity). From this it follows that the energy of the total problem is exactly given by the sum of the energies of the effective $1+1$ problems. Section 3.2 completes the analysis with the study of energy fluxes through the (null) boundaries

$$
F=\sum_{\ell m} \gamma \partial_{\tau} \phi_{\ell m} \partial_{\tau} \bar{\phi}_{\ell m}
$$

$\ddagger$ We dwell in a finite-dimensional setting. See [25 97] for the discussion in the infinite-dimensional case. Also, in this abstract discussion of the pseudospectrum, we use $\lambda$ to denote eigenvalues, reserving $\omega$ for the case where they are actually frequencies in the spectrum of a time generator $L$, as in eq. (4) obtained by Fourier transform of eq. (1). 
accounted in terms of the function $\gamma$ in the $L_{2}$ operator in (3). Such operator $L_{2}$ is therefore neatly identified with the non-conservative character of the system and its non-selfadjointness.

The discussion of the energy scalar product and energy norm is presented in section 3.2 .

\subsection{Energy scalar product: applications}

Once the basic elements of the scalar product and its associated norm have been presented in sections 3.1 and 3.2, we make use of them in some applications in sections 4.1 and 4.2 .

2.2.1. QNM weak formulation and finite element calculations. Using the scalar product, the eigenvalue problem (4) can be cast in a weak formulation (cf. section 4.1, equation (108)

$$
\int_{a}^{b} W_{L}\left(u_{T}, u\right) d x=i \omega \int_{a}^{b} W_{R}\left(u_{T}, u\right) d x,
$$

in terms of appropriate bilinear forms $W_{L}\left(u_{T}, u\right)$ and $W_{R}\left(u_{T}, u\right)$ and the $u_{T}$ test functions. This is by itself an interesting perspective on the QNM problem, in particular when aiming at identifying the appropriate functional space where QNMs are defined or when systematically studying the effect of random perturbations on the distribution of resonant frequencies, seen as eigenvalue values [97]. As a practical application, such a weak formulation is employed here to study the possibility of a numerical artifact behind the observed QNM instability, namely arising from the employed numerical scheme in [53, 54, 26], based on Chebyshev spectral methods. This point specifically addresses one of the caveats raised in [53], in relation to the adopted (numerical) analysis scheme. In concrete terms, the weak formulation (11) permits to implement a completely independent numerical setting, namely a finite elements scheme. The results presented here confirm the presence of Nollert-Price-like QNM branches described in [53], demonstrating the independence of the latter from the Chebyshev's spectral scheme.

2.2.2. QNM resonant expansions: a Keldysh's projection expression A second application, presented in section 4.2, concerns resonant expansions. One of the key features of the hyperbolic formulation is that QNM are normalizable, in particular eigenfunctions live in a Banach (actually a Hilbert) space. This permits to make use of Keldysh's theorem for the expansion for the resolvent of an operator in a Banach space [59, 60, 78, 13, 14]. Specifically, associated with the introduced energy scalar product, we have the adjoint $L^{\dagger}$ of $L$, so we can complete (4) with the left-eigenvalue problem (namely the right-eigenvalue problem of $L^{\dagger}$ )

$$
L \hat{v}_{n}=\omega_{n} \hat{v}_{n}, L^{\dagger} \hat{w}_{n}=\bar{\omega}_{n} \hat{w}_{n},
$$

where $\hat{v}_{n}$ and $\hat{w}_{n}$ are, respectively right- and left-eigenvectors normalized in the energy norm. The application of Keldysh's theorem (under the assumption of a spectrum given only by eigenvalues) then leads to the following expansion of the scattered field $u$ in terms of QNMs

$$
u(\tau, x) \sim \sum_{n} e^{i \omega_{n} \tau} a_{n} \hat{v}_{n}(x)
$$

with coefficients $a_{n}$ obtained by projection on initial data $u_{0}$ for the equation (1)

$$
a_{n}=\kappa_{n}\left\langle\hat{w}_{n} \mid u_{0}\right\rangle_{E},
$$

with $\kappa_{n}$ the associated condition numbers (see their general definition in equation (128)

$$
\kappa_{n}=\frac{1}{\left\langle\hat{w}_{n}, \hat{v}_{n}\right\rangle_{E}} .
$$


This expression presents the following features:

i) The QNM expansion is, in the generic case, a (non-convergent) asymptotic series (cf. discussion around equation (152) for details).

ii) In a hyperboloidal slicing scheme, it provides an explicit expression for the QNM (asymptotic) expansion coefficients in terms of initial data, even if $\hat{v}_{n}$ do not form a complete set, providing a prescription for a frequency-domain solution to equation (1).

iii) It reduces to the standard form in the selfadjoint (in general, normal) case, where $\kappa_{n}=1$ and $\hat{w}_{n}=\hat{v}_{n}$. In this case, the series is actually convergent and the set $\left\{\hat{v}_{n}\right\}$ is a (Hilbert) orthonormal basis. In particular, it recovers the explicit expression for normal modes.

\subsection{Energy scalar product: general picture, heuristics and geometry.}

Sections 5 and 6 have a more heuristic flavor, aiming at framing on a geometric setting some of the open conceptual problems in the approach to QNM instability, built on the hyperboloidal approach to scattering and the pseudospectrum, as introduced in reference [53].

2.3.1. Building a general picture of QNM ultraviolet instability. Section 5 presents a description of the generic picture of ultraviolet QNM instability as emerging from the results in [53] and [54]. Specifically, QNM overtones of non-perturbed black holes are structurally unstable under high-frequency perturbations, migrating to new QNM branches when perturbed. The resulting perturbed branches, on the contrary, are themselves structurally stable, that is, QNM stay in the perturbed branches if further perturbed.

Such new QNM branches, referred to generically as Nollert-Price QNM branches, display open patterns in the complex plane pushed by perturbations closer to the real axial (increase of damping) and without upper bounds in the real part (therefore exploring small spacetime structures). Nollert-Price branches are constrained to lay in complex plane regions bounded below by QNM-free regions - determined from the boundaries of $\epsilon$ pseudospectra - that present asymptotic logarithmic boundaries for large real parts

$$
\operatorname{Im}(\omega) \sim C_{1}+C_{2} \ln \left(|\operatorname{Re}(\omega)|+C_{3}\right),|\operatorname{Re}(\omega)| \gg 1
$$

Qualitative different Nollert-Price-like branches appear under perturbations, possibly associated with different underlying resonance mechanisms. Transition between such distinct regimes are marked by the presence of "inner QNMs", much closer to the imaginary axis. Finally, the slowest decaying QNM is stable under the considered ultraviolet perturbations.

2.3.2. BH spectroscopy: $\epsilon$-dual QNM expansions. As a by-product of combining QNM instability and QNM resonant expansions, section 5.3 introduces the notion of $\epsilon$-dual QNM expansions of a (time-domain) scattered signal, of potential use in $\mathrm{BH}$ spectroscopy.

BH spectroscopy [11, 27, 6, 82, 49, 38, 48, 18, 76, 83] aims at retrieving the physical information of a $\mathrm{BH}$ from the expansion of the ringdown gravitational wave signal $(\mathrm{GW})$ in terms of QNMs. In the present setting, this amounts to expand the scattered field $u(\tau, x)$ in equation (1) in terms of QNMs, according to equation (13). If we consider now an astrophysical $\mathrm{BH}$ subject to small perturbations, modeled by operator perturbations with $\|\delta L\|=\epsilon$, the resulting perturbed scattered field $u^{\epsilon}(\tau, x)$ that solves equation (1) with $L+\delta L$, admits rather a QNM expansion in terms of the corresponding perturbed QNMs in (5)

$$
u^{\epsilon}(\tau, x) \sim \sum_{n} e^{i \omega_{n}^{\epsilon} \tau} a_{n}^{\epsilon} \hat{v}_{n}^{\epsilon}(x)
$$


Given the QNM spectral instability under ultraviolet perturbations described in [53, 54], perturbed QNM frequencies $\omega_{n}^{\epsilon}$ can strongly differ from QNM frequencies $\omega_{n}$, corresponding to the non-perturbed BH. The presence of such perturbed QNMs in the perturbed scattered signal, as well as the need to expand in terms of perturbed QNMs in order to reach arbitrary accuracy of the resonant expansion, have been demonstrated in [54]. This raises the following question: are QNM expansions of GW signals in terms of non-perturbed QNMs meaningful?

Remarkably, the answer is in the affirmative, leading to the notion of $\epsilon$-dual QNM expansions. Such notion follows from combining: i) the instability of the (frequency-domain) spectral QNM problem (4), and ii) the stability of the (time-domain) evolution problem (1). Such $\epsilon$-dual QNM expansions are very different resonant expansions of "essentially the same scattered waveform". More precisely, they are either resonant expansions of a time-domain signal $u(\tau, x)$ in terms of the standard non-perturbed QNMs $\omega_{n}$-namely expansion (13) or, alternatively, a expansion of $u^{\epsilon}(\tau, x)$ in terms of the (very different) perturbed QNMs $\omega_{n}^{\epsilon}$ -namely expansion (17). From the dynamical stability of the evolution problem (1), one has $u^{\epsilon}(\tau, x) \sim u(\tau, x)+O(\epsilon)$, so that the respective QNM expansions cannot be distinguished at the order $\epsilon$. In other words, they are equivalent at this order $\epsilon$, something we note as

$$
\sum_{n} e^{i \omega_{n}^{\epsilon} \tau} a_{n}^{\epsilon} \hat{v}_{n}^{\epsilon}(x) \stackrel{\epsilon}{\sim} \sum_{n} e^{i \omega_{n} \tau} a_{n} \hat{v}_{n}(x)
$$

Both expansions approach the actual astrophysical (i.e. the perturbed) waveform, the one on the left with arbitrary accuracy, the one on the right to order $O(\epsilon)$. But for all practical purposes (the errors in the observational GW data are much bigger than $\epsilon$ ) both are valid.

Which expansion should then be chosen? From our perspective, and if technically possible, both of them. Indeed, they contain complementary information. Whereas the standard expansion in terms of non-perturbed $\omega_{n}$ QNMs encodes information of the large scale structures of the (averaged) BH, the expansion in terms of perturbed $\omega_{n}^{\epsilon}$ informs on the small perturbation scales [54]. Because of this complementarity we refer to them as " $\epsilon$-dual".

2.3.3. Operator norm and metric fluctuations. The previous emerging picture is of descriptive nature, without discussing the underlying physical and geometrical phenomena. A first key point is discussed in section 6.1, namely the fact that, when considering perturbations with a given "energy norm" $\epsilon$ in the QNM instability problem, such $\epsilon$ is not the actual energy of a spacetime perturbation $\delta g_{a b}$. It corresponds rather to the "size" $\|\delta L\|_{E}$ of the perturbation of the operator $L$ generating evolution in equation (1). Such a quantity is indeed related to the "energy" (norm) of spacetime perturbations $\delta g_{a b}$, but such a relation is not a simple one.

The discussion in section 6.1 leads to a "pointwise" estimation of $\|\delta L\|_{E}$ in terms of the energy density of $\delta g_{a b}$ (understood as a field perturbation on a fixed background). More specifically, $\|\delta L\|_{E}$ is estimated by the maximum of (compact support) perturbations $\delta \tilde{V}$ to the effective potential in equation (84), namely

$$
\|\delta L\|_{E} \sim \max _{\operatorname{supp}(\delta \tilde{V})}|\delta \tilde{V}| .
$$

On the other side, the variations $\delta \tilde{V}$ are of the order of second derivatives of the metric perturbations, $\partial^{2} \delta g_{a b}$, this leading to $\delta \tilde{V}$ being estimated by an energy density of the perturbations $\rho_{E, \delta g}(x) \sim\left|\partial \delta g_{a b}\right|^{2}$, so we can crudely write

$$
\left.\left|\delta g_{a b}\right||| \delta L\right|_{E} \sim \max _{\operatorname{supp}(\delta g)}\left|\partial \delta g_{a b}\right|^{2} \sim \max _{\operatorname{supp}(\delta g)} \rho_{E}(\delta g ; x) .
$$


This pointwise $\left(L^{\infty}\right.$-like) expression indicates that when estimating the perturbations $\delta L$ triggering QNM instability, the right quantity to consider is not the total energy of the perturbations $\delta g_{a b}$, but the peaks of their spacetime energy distribution. In other words: given two spacetime perturbations $\delta g_{a b}$ with the same energy $E=\int_{\operatorname{supp}(\delta g)} \rho_{E, \delta g}(x) d x$, the one impacting more strongly the QNM instability is the one with a larger maximum in $\rho_{E, \delta g}(x)$.

From a physical perspective the conclusion is that spiked distributions of spacetime perturbations are more efficient in triggering QNM instabilities than smooth distributions. Astrophysically, clouds of compact objects would be more efficient than clouds of gas.

\subsubsection{QNM instability and compact object QNM universality: from high-frequency Burnett's} conjecture to a Regge-QNM's conjecture. As discussed in section 5 (cf. also 2.3.1 above), ultraviolet perturbations push QNMs toward logarithmic curves 16, , getting closer and closer as the norm or the frequency of the perturbation increase, but always laying above them as long as the spacetime perturbations are smooth. A question is posed: do Nollert-Price branches reach logarithmic pseudospectra boundaries in the limit of infinite frequency?

Reference [54] conjectures that this is the case, based on numerical considerations. In section 6.2, a heuristic avenue to support this conjecture and to systematically address this question is proposed based on the so-called Burnett's conjecture [17] on the high-frequency limit of spacetimes. Burnett's conjecture states that the limit of high-frequency oscillations of a vacuum spacetime is effectively described by an effective matter spacetime described by massless Einstein-Vlasov. However, Burnett's original approach does not allow for spacetime "concentrations" (spikes), the key feature of spacetime perturbations for efficiently triggering instabilities, according to expression (20). A refinement of the high-frequency limit scheme, allowing for concentrations, has been presented by Luk \& Rodnianski [74]. The main outcome of such analysis, for the considerations our present setting, is that the high-frequency spacetime limit to $g_{\infty}$ is of low-regularity nature, namely

$$
g_{n} \rightarrow g_{\infty} \text { in } C^{0}, \partial g_{n} \rightarrow \partial g_{\infty} \text { in } L^{2}
$$

Putting this together with the results [90, 113] (see also [8]), stating that QNMs of lowregularity $C^{p}$ potentials have a universal structure in terms of so-called Regge (broad) resonances, we formulate the QNM high-frequency limit conjecture in [54] as :

Under generic ultraviolet perturbations and in the limit of infinite frequency, $B H$ Nollert-Price QNM branches become Regge QNM branches, presenting the $n \gg 1$ asymptotics

$$
\begin{aligned}
& \operatorname{Re}\left(\omega_{n}\right) \sim \pm\left(\frac{\pi}{L} n+\frac{\pi \gamma_{p}}{2 L}\right) \\
& \operatorname{Im}\left(\omega_{n}\right) \sim \frac{1}{L}\left[\gamma \ln \left(\left(\frac{\pi}{L} n+\frac{\pi \gamma_{p}}{2 L}\right)+\frac{\pi \gamma_{\Delta}}{2 L}\right)-\ln S\right] .
\end{aligned}
$$

This conjecture states not only that Nollert-Price QNMs asymptote to logarithmic lines in the infinite high-frequency limit, but also proposes the specific pattern that QNMs follow on those logarithmic curves. This connects with the asymptotics of $w$-modes of matter compact objects [61, 112], enforcing the idea proposed in [53] that (w-)QNM asymptotics of generic (vacuum or matter) astrophysical compact objects are universal in the high-frequency limit.

2.3.5. Towards a geometric formulation of QNM instability. In their current form, all discussions above depend strongly on a particular choice of coordinates in the hyperboloidal approach. This could be an obstacle when trying to extend such analyses to generic situations (e.g. beyond spherical symmetry), but also can obscure their underlying invariant content. 
Section 6.3 aims at laying the basic elements to build a genuinely geometric construction of the approach. The main elements ingredients can be summarized in the following points:

i) Foliations of the null boundaries. The basic starting point would be the choice of respective foliations $\left\{\mathcal{S}_{\tau}^{\infty}\right\}$ of null infinity $\mathscr{I}^{+}$and $\left\{\mathcal{S}_{\tau}^{\mathcal{H}}\right\}$ of the BH horizon $\mathcal{H}$, together with a function $\gamma$ defined on sections $\mathcal{S}_{\tau}^{\infty}$ and $\mathcal{S}_{\tau}^{\mathcal{H}}$ that controls the energy flux in equation (10). These objects are arbitrary, but are kept fixed. Then, the boundary foliations are extended to the bulk in a spacetime (hyperboloidal) foliation $\left\{\Sigma_{\tau}\right\}$ that "interpolates" between $\left\{\mathcal{S}_{\tau}^{\infty}\right\}$ and $\left\{\mathcal{S}_{\tau}^{\mathcal{H}}\right\}$. The resulting slices $\Sigma_{\tau}$ are then compactified. Such bulk extension and the subsequent compactification are (essentially) arbitrary.

ii) QNMs in a geometric approach. Expressions for $L_{1}$ and $L_{2}$ in equations (3) are promoted to a geometric form 225 on $\Sigma_{\tau}$, and boundaries $\mathcal{S}_{\tau}^{\infty}$ and $\mathcal{S}_{\tau}^{\mathcal{H}}$ are "shrinked" to points $i^{\infty}$ and $i^{\mathcal{H}}$, respectively. Slices $\Sigma_{\tau}$ become 3 -spheres $\mathbb{S}^{3}$ "pinched" at $i^{\infty}$ and $i^{\mathcal{H}}$. In this setting, $L_{1}$ is promoted to an operator $\stackrel{\circ}{L}_{1}$ acting on a "completed" 3 -sphere, whereas $L_{2}$ acts on the pinched 3 -sphere. No boundary conditions enter through $L_{1}$ (that acts on a closed manifold) and, regarding $L_{2}$, only the value of $\gamma$ at the pinching holes is relevant. Under these conditions, the QNM spectrum would become automatically discrete.

iii) "Missing" degrees of freedom and BMS symmetry. Denoting by $\ell^{a}$ the null generators of $\mathscr{I}^{+}$and $\mathcal{H}$, vector fields on such null boundaries satisfying

$$
\xi^{a}=\gamma \ell^{a}, \mathcal{L}_{\ell} \gamma=0
$$

can be associated with BMS supertranslations (in $\mathcal{H}$ this is an abuse of language). Such symmetries would relate the different possible choices of boundary foliations in the point i) above, restoring invariance to the construction. On the other hand, understanding BMS as a dynamical symmetry generating a phase space of degrees of freedom at the null boundaries —or, in another language, understanding BMS as (boundary residual) broken symmetry enlarging the physical phase space- outgoing bulk degrees of freedom $\phi$ would interact with "pinching-BMS" degrees of freedom $\gamma$ at $i^{\infty}$ and $i^{\mathcal{H}}$, with a coupling fixed in terms of the flux term (10). In particular, $\stackrel{\circ}{L}_{1}$ would be a bulk operator oblivious to pinching holes, whereas $L_{2}$ would be essentially a "pinching-BMS" operator.

The extension of the phase space of degrees of freedom with such "boundary data" could provide clues into QNM instability from an "inverse scattering" perspective and, in parallel, could offer an avenue to restore selfadjointness into the full problem, leading to a notion of global spacetime normal modes in the classical formulation of the problem.

\section{QNM spectral instability, pseudospectrum and scalar product choice}

In this section we discuss the question of the choice of scalar product - and its associated norm - in the study of the spectral stability properties of an operator. First, we illustrate the impact of such a choice with a simple example. Second, we present the spacetime construction of the energy scalar product proposed in [53] for the hyperboloidal approach to QNMs.

\subsection{Spectral instability as a norm-dependent notion: a study case}

We consider a simple linear second order differential operator — with constant coefficientsand study the role of the choice of scalar product in the assessment of selfadjointness and normality. Once this is done, we display the consequence of such choices in its eigenvalue stability problem, by using the pseudospectrum. 
Although this calculation is elementary, we find it illustrative for its explicit character. Additionally, it exhibits, in a very simple setting, the difference between a formally normal operator and an actual normal operator, key in the functional context of QNM instability.

\subsubsection{Scalar product, selfadjointness and normality. Let $Q$ be the operator}

$$
Q=a \frac{d^{2}}{d x^{2}}+b \frac{d}{d x}+c,
$$

where $a, b, c \in \mathbb{R}$, with $a \neq 0$, acting on functions defined on the interval $x \in\left[x_{0}, x_{1}\right]$. To complete the definition of $Q$ we must specify is domain $\mathcal{D}$, i.e. the functional space in which its acts. Let us illustrate the involved issues in different points:

i) Square-integral functions: assessing self-adjointness. First we consider the standard space of square-integrable functions $L^{2}\left(\left[x_{0}, x_{1}\right], d x\right)$, with scalar product

$$
\langle\varphi, \phi\rangle_{2}=\int_{x_{0}}^{x_{1}} \bar{\varphi} \phi d x
$$

A direct calculation using integration by parts shows that

$$
\langle\varphi, Q \phi\rangle_{2}=\left.\left(a\left(\bar{\varphi} \phi^{\prime}-\phi \bar{\varphi}^{\prime}\right)+b \bar{\varphi} \phi\right)\right|_{x_{0}} ^{x_{1}}+\left\langle Q_{2}^{\dagger} \varphi, \phi\right\rangle_{2},
$$

where $Q_{2}^{\dagger}$ is given by

$$
Q_{2}^{\dagger}=a \frac{d^{2}}{d x^{2}}-b \frac{d}{d x}+c
$$

If we now impose homogeneous Dirichlet boundary conditions, we can write

$$
\langle\varphi, Q \phi\rangle_{2}=\left\langle Q^{\dagger} \varphi, \phi\right\rangle_{2}
$$

and $Q_{2}^{\dagger}$ is the "formal adjoint" of $Q$-the subscript 2 stresses that this result depends on the employed scalar product $\langle\cdot, \cdot\rangle_{2}$, as it will be further discussed below. A symmetric operator is characterised by

$$
\langle\varphi, Q \phi\rangle=\langle Q \varphi, \phi\rangle
$$

and, from equations (24) and (27), this happens for $Q$ when $b=0$. If $b \neq 0$ the operator $Q$ is not symmetric and, therefore, cannot be selfadjoint. For $b=0, Q$ is selfadjoint if, in addition, the domains of $Q$ and $Q_{2}^{\dagger}$ coincide, something we can achieve by imposing $\mathcal{D}(Q)=\mathcal{D}\left(Q_{2}^{\dagger}\right)=\mathcal{D}$ with

$$
\mathcal{D}=\left\{\phi \in L^{2}\left(\left[x_{0}, x_{1}\right], d x\right) \mid \phi\left(x_{0}\right)=\phi\left(x_{1}\right)=0\right\},
$$

the $Q$ is a selfadjoint operator $[$ In sum, for homogeneous Dirichlet (more generally, homogeneous Robin) boundary conditions, $Q$ is selfadjoint if $b=0$. In our setting, this translates in the fact that $Q$ is spectrally stable for $b=0$ and there is no guarantee of spectral stability for $b \neq 0$. This is indeed confirmed numerically: under small randon perturbations $\delta Q$ of small size $\|\delta Q\|_{2}=\epsilon$ the eigenvalues for $b=0$ changes linearly in $\epsilon$, whereas in the non-selfadjoint case $b \neq 0$ we find indeed spectral instability.

$\S$ As pointed out above, if the domains $\mathcal{D}(Q)$ and $\mathcal{D}\left(Q_{2}^{\dagger}\right)$ are not specified one refers to $Q_{2}^{\dagger}$ as the formal adjoint of $Q$ and, if 28 is satisfied, then $Q$ is formally selfadjoint. 
ii) Normality and "formal normality". The non-selfadjoint case $b \neq 0$ presents an apparent catch. Spectral stability is guaranteed for a larger class than selfadjoint operators. Normal operators — namely operators that commute with their adjoints — also satisfy a spectral theorem guaranteeing spectrally stability. In our case, because of the constancy of $a, b$ and $c$ in equations 24) and 28, operators $Q$ and $Q_{2}^{\dagger}$ actually satisfy

$$
\left[Q, Q_{2}^{\dagger}\right]=Q Q_{2}^{\dagger}-Q_{2}^{\dagger} Q=0 \Longleftrightarrow Q Q_{2}^{\dagger}=Q_{2}^{\dagger} Q
$$

In spite of this, the operator $Q$ for $b \neq 0$ is indeed spectrally unstable (in the $\|\cdot\|_{2}$ norm, see later): how does this reconcile with $Q$ satifying (31) for any $b$ ?

The way out of this apparent contradiction is that $Q$ satisfying (31) only qualifies it as "formally normal". In order to conclude actual normality (for which spectral stability holds) for $Q$, one needs to verify that the domains $\mathcal{D}\left(Q Q_{2}^{\dagger}\right)$ and $\mathcal{D}\left(Q_{2}^{\dagger} Q\right)$ coincide, so that operators $Q Q_{2}^{\dagger}$ and $Q_{2}^{\dagger} Q$ are indeed the same. Such domains are characterised as

$$
\begin{aligned}
& \mathcal{D}\left(Q Q_{2}^{\dagger}\right)=\left\{\phi \in \mathcal{D}\left(Q_{2}^{\dagger}\right) \mid Q_{2}^{\dagger} \phi \in \mathcal{D}(Q)\right\} \\
& \mathcal{D}\left(Q_{2}^{\dagger} Q\right)=\left\{\phi \in \mathcal{D}(Q) \mid Q \phi \in \mathcal{D}\left(Q_{2}^{\dagger}\right)\right\}
\end{aligned}
$$

Since $\mathcal{D}(Q)=\mathcal{D}\left(Q_{2}^{\dagger}\right)=\mathcal{D}$, unwrapping the definitions leads to

$$
\begin{aligned}
& \mathcal{D}\left(Q Q_{2}^{\dagger}\right)=\left\{\phi \in \mathcal{H}_{2} \mid \phi\left(x_{0}\right)=\phi\left(x_{1}\right)=0, \phi^{\prime \prime}\left(x_{0}\right)=a^{-1} b \phi^{\prime}\left(x_{0}\right), \phi^{\prime \prime}\left(x_{1}\right)=a^{-1} b \phi^{\prime}\left(x_{1}\right)\right\} \\
& \mathcal{D}\left(Q_{2}^{\dagger} Q\right)=\left\{\phi \in \mathcal{H}_{2} \mid \phi\left(x_{0}\right)=\phi\left(x_{1}\right)=0, \phi^{\prime \prime}\left(x_{0}\right)=-a^{-1} b \phi^{\prime}\left(x_{0}\right), \phi^{\prime \prime}\left(x_{1}\right)=-a^{-1} b \phi^{\prime}\left(x_{1}\right)\right\} .
\end{aligned}
$$

Hence, if $b \neq 0$, we can conclude

$$
\mathcal{D}\left(Q Q_{2}^{\dagger}\right) \neq \mathcal{D}\left(Q_{2}^{\dagger} Q\right)
$$

Therefore, if $b \neq 0$, then $Q$ is not normal due to the mismatch of the domains: it is only formally normal. This restores consistency with the observed spectral instability when using $\|\cdot\|_{2}$ to measure the size of perturbations.

iii) The crucial role of the scalar product: a full reassessment of selfadjointness and spectral stability. But there is still a further twist with the $Q$ operator in equation (24): this operator is actually selfadjoint for all values $a, b$ and $c$, if the scalar product is chosen in the appropriate way. In particular, for $b \neq 0$, there is a choice of scalar product for which $Q$ is not only normal — and therefore normal and spectrally stable — but actually

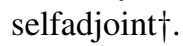

This follows from the fact that any linear differential second-order operator in one dimension can be written in Sturm-Liouville form by using an appropriate "integrating factor" $w(x)$. The latter can the be used to build the scalar product $\langle\cdot, \cdot\rangle_{w}$, that makes the original operator actually selfadjoint. To see this in our particular case, let us consider the function $w(x)=a^{-1} \exp \left(a^{-1} b x\right)$, with $x \in\left[x_{0}, x_{1}\right]$. Then using this integrating factor, $Q$ can be rewritten in the Sturm-Liouville form

$$
Q=\frac{1}{w(x)}\left(\frac{d}{d x}\left(p(x) \frac{d}{d x}\right)-q(x)\right),
$$

$\dagger$ We thank Graham Cox for pointing out this fact, that makes the example of the operator $Q$ in equation 24 even richer than we had originally noticed. 
where $p(x)=a w(x)$ and $q(x)=-c w(x)$. Then, defining the scalar product

$$
\langle\varphi, \phi\rangle_{w}=\int_{x_{0}}^{x_{1}} \bar{\varphi} \phi w(x) d x,
$$

and integrating by parts one obtains

$$
\langle\varphi, Q \phi\rangle_{w}=\left.\left(p\left(\bar{\varphi} \phi^{\prime}-\phi \bar{\varphi}^{\prime}\right)\right)\right|_{x_{0}} ^{x_{1}}+\left\langle Q_{w}^{\dagger} \varphi, \phi\right\rangle_{w}
$$

where $Q_{w}^{\dagger}=Q$. Thus, if we impose homogeneous Dirichlet (again, more generally, homogeneous Robin) boundary conditions

$$
\mathcal{D}=\left\{\phi \in\left(L^{2}\left(\left[x_{0}, x_{1}\right], w(x) d x\right) \mid \phi\left(x_{0}\right)=\phi\left(x_{1}\right)=0\right\},\right.
$$

one concludes that $Q$ is a selfadjoint operator.

If we focus now in the case $b \neq 0$, how can we reconcile the fact that $Q$ is non-normal and spectrally unstable when using the norm $\|\cdot\|_{2}$, while selfadjoint and therefore spectrally stable consider in the norm $\|\cdot\|_{w}$ ? Then answer is that a given perturbation $\delta Q$ can have a small 2-norm $\|\delta Q\|_{2}$ while having a large $w$-norm $\|\delta Q\|_{w}$. In the former case, a significant change in the eigenvalues is interpreted as a spectral instability, whereas in the latter case the large change in the spectrum is consistent with stability, since the operator perturbation is also large. This provides a neat illustration of the important of assessing "big" and "small" through the norm, when discussing spectral instability. In the next subsection we address this point from the point of view of the pseudspectrum.

3.1.2. QNM stability through the pseudospectrum: the role of the scalar product. As discussed in section 2.1.1, the notion of pseudospectrum provides an avenue to assess spectral instability. Here we discuss how the choice of the scalar product impacts the structure of the pseudospectrum, illustrating it with the operator $Q$ and scalars products dicussed above.

Following the systematic presentation in [64] (cf. also the discussion in [53] in the present gravitational setting) the pseudospectrum consists, by definition, of nested sets in the complex plane around the eigenvalues of the operator. The pseudospectrum allows to measure how much a perturbations to the operator affect its eigenvalues. As a consequence of the Bauer-Fike theorem [64], the eigenvalues of a selfadjoint operator are stable in the sense that a change $\delta Q$ of "size" $\epsilon$ in the operator result in a change of the same order $\epsilon$ in the eigenvalues. As a consequence, graphically, selfadjoint operators have a "flat pseudospectrum" (see discussion in [53]). Notice that although the eigenvalues of an operator are independent of the chosen norm $\|\cdot\|$, the notion of pseudospectrum is not, since determining the size of the perturbation $\|\delta Q\|$ depends intimately on the choice for $\|\cdot\|$. This is particularly important for physical applications where one is interested in small perturbations where the norm should correspond to a physically relevant notion of energy for the problem.

The latter point is illustrated in the following through the construction of the pseudospectrum of the operator $Q$, computed numerically using spectral methods, by employing the two norms induced by the inner products $\langle\cdot, \cdot\rangle_{2}$ and $\langle\cdot, \cdot\rangle_{w}$, respectively introduced in equations (25) and (37). To be more precise, we consider the spectral stability of a related operator $\hat{Q}$, whose eigenvalue problem is closer to the ones studied in [53] in QNM context. Specifically, starting from the eigenvalue for $Q$

$$
Q \Phi=\lambda \Phi,
$$


we define the rescaled variable $\Phi=-\left(x-x_{0}\right)\left(x-x_{1}\right) \phi$. This incorporates the boundary conditions into the operator $\$$ Specifically, requiring boundedness for $\phi$ at $x_{0}$ and $x_{1}$, encodes imposing homogeneous Dirichlet boundary conditions for $\Phi$. Then, we can write the eigenvalue problem (40) as $\S$

$$
\hat{Q} \phi=\lambda \phi
$$

where,

$$
\hat{Q}=A(x) \frac{d^{2}}{d x^{2}}+B(x) \frac{d}{d x}+C(x)
$$

with

$$
\begin{aligned}
& A(x)=-a\left(x-x_{0}\right)\left(x-x_{1}\right) \\
& B(x)=-b\left(x-x_{0}\right)\left(x-x_{1}\right)+2 a\left(-2 x+x_{0}+x_{1}\right) \\
& C(x)=-2 a-c\left(x-x_{0}\right)\left(x-x_{1}\right)+b\left(-2 x+x_{0}+x_{1}\right) .
\end{aligned}
$$

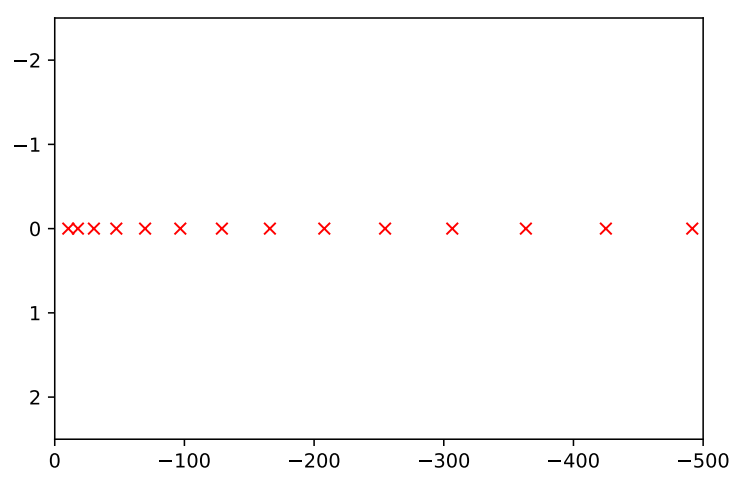

Figure 1. Numerically computed eigenvalues for the operator $\hat{Q}$ with $x_{0}=-1, x_{1}=1$ and $a=1, b=6, c=1$ with a spatial discretization $N=100$ points

We illustrate the impact of the choice scalar product and its associated norm in the pseudospectrum of the operator $\hat{Q}$, by computating it numerically. The starting point is its second characterization in equation (7), in a discretized version of the operators and scalar product (details are given in Appendices B and C in [53]). In particular, given a scalar product $\langle\cdot, \cdot\rangle_{G}$ and two functions $\varphi, \phi$, the discretized version of their scalar product can be written as $\langle\varphi, \phi\rangle_{G}=\left(\varphi^{*}\right)^{i} G_{i j} \phi^{j}$ where $G_{i j}$ is the Gram matrix associated with the scalar product and $*$ is the conjugate-transpose (note the abuse of notation with $\varphi, \phi \in \mathbb{C}^{n}$ also denoting the discretized version of the functions). The discretised adjoint $\hat{Q}^{\dagger}$ is then written in terms of the Gram matrix as $\hat{Q}^{\dagger}=G^{-1} \hat{Q}^{*} G$. With these elements, it can be shown [105, 53] that the

$\ddagger$ This is in the same spirit that outgoing boundary conditions in the hyperboloidal approach [3, 85, 84, 53] are incorporated into the operator by choosing a slicing intersects the BH horizon and null infinity.

$\S$ From the discussion in 3.1.1 we know that there exists a scalar product - that associated to the Sturm-Liouville form of $Q$ - for which the operator $Q$ is selfadjoint — a completely analogous calculation can be done for $\hat{Q}$ - hence we know $Q$ must have real eigenvalues. One can can numerically corroborate this fact. A numerical implementation in python using the linalg package for linear algebra in numpy, and using Chebyshev spectral methods to approximate the derivatives — see [102]53] for further discussion on spectral methods - renders Figure 1 
$\sigma_{G}^{\epsilon}(\hat{Q})$ pseudospectrum associated with the scalar product $\langle\cdot, \cdot\rangle_{G}$ is characterised as

$$
\sigma_{G}^{\epsilon}(\hat{Q})=\left\{\lambda \in \mathbb{C}: s_{G}^{\min }(\lambda \mathrm{Id}-\hat{Q})<\epsilon\right\},
$$

where $s_{G}^{\min }(M)=\min \left\{\sqrt{\lambda}: \lambda \in \sigma\left(M^{\dagger} M\right)\right\}$, is the minimum of the singular values of the matrix $M$ (in a generalised version associated adjoints respect to $\langle\cdot, \cdot\rangle_{G}$ ).

We are now in condition of calculating the pseudospectra $\sigma_{2}^{\epsilon}(\hat{Q})$ and $\sigma_{w}^{\epsilon}(\hat{Q})$ associated, respectively to the standard $L^{2}$ scalar product (25) and the Sturm-Liouville-like (37). The former produces the pseudospectrum shown in Figure 2 for the operator $\hat{Q}$. As one can observe in Figure 2, using the $L^{2}$-inner product renders a "non-flat" pseudospectrum for $\hat{Q}$. Note the range of values of $\epsilon$ : the borad extension of pseudospectra sets with small $\epsilon$ indicates spectral instability.

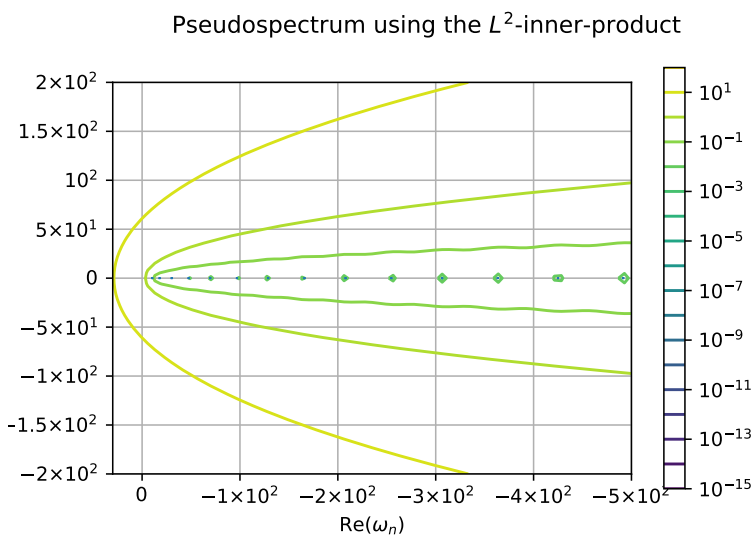

Figure 2. Numerically computed pseudospectrum for the operator $\hat{Q}$ with $x_{0}=-1, x_{1}=1$ and $a=1, b=6, c=1$ with a spatial discretization $N=100$ points. The standard matrix norm inherited from the standard $L^{2}$-inner product was used. One can observe that although the pseudospectrum consists of nested sets around the eigenvalues of $\hat{Q}$ it is not flat: the $\epsilon$ contour lines open up as one considers eigenvalues with larger modulus (overtones).

Regarding $\sigma_{w}^{\epsilon}(\hat{Q})$, the key information is encoded in the integration factor $w(x)$ associated to the Sturm-Liouville form of the $\hat{Q}$ operator, namely

$$
w(x)=A(x)^{-1} \exp \left(\int_{x} A\left(x^{\prime}\right)^{-1} B\left(x^{\prime}\right) d x^{\prime}\right) .
$$

Explicitly for the present case, substituting the polynomials of equation (43) into equation (45) and integrating, renders

$$
w(x)=-\frac{\left(x-x_{0}\right)\left(x-x_{1}\right)}{a} \exp \left(\frac{b\left(x-x_{0}\right)}{a}\right) .
$$

The numerical Chebyshev spectral methods implementation of the Gram matrix associated to the scalar product in $\langle\cdot, \cdot\rangle_{w}$ in equation (37), following the discussion in Appendix $\mathrm{C}$ of [53], gives the pseudospectrum shown in Figure 3 As one can observe in Figure 3 the pseudospectrum of $\hat{Q}$ is "flat" as expected, with concentric circles around eigenvalues, and a completely different range of $\epsilon$ 's with large $\epsilon$ ' in pseudospectra extending far from the 
eigenvalues: this indicates stability as it corresponds to a selfadjoitn operator. The contrast between Figures 2 and 3 shows, in a controlled set up, that given an operator, the choice of inner product and associated norm —with their corresponding (different) notions of big and small - play a fundamental role for the assessment of spectral stability: an operator can be stable in one norm and unstable in another. The proper choice of scalar product is therefore critical.

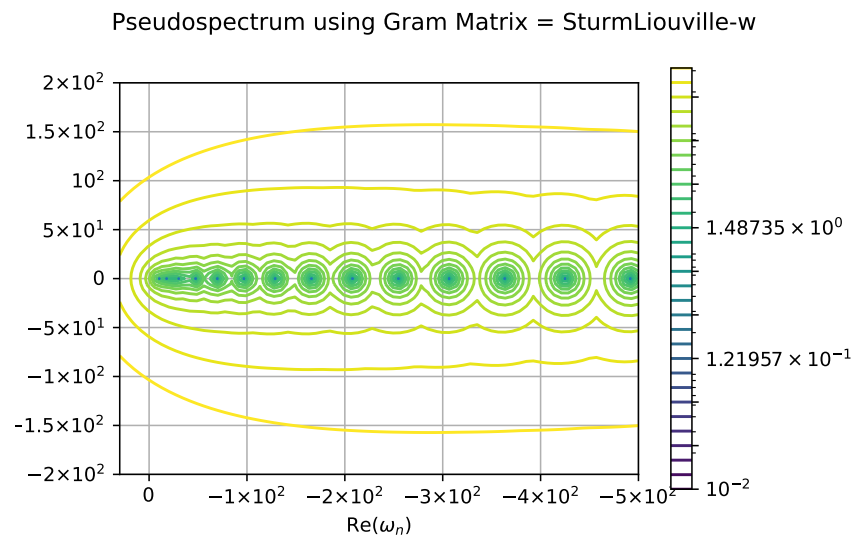

Figure 3. Numerically computed pseudospectrum for the operator $\hat{Q}$ with $x_{0}=-1, x_{1}=1$ and $a=1, b=6, c=1$ with a spatial discretization $N=100$ points. The Gram matrix associated to the Sturm-Liouville inner product was used. One can observe that with this inner product, the pseudospectrum of $\hat{Q}$ is flat, indicating stability which is consistent with the selfadjointness of this operator respect to the inner product 37

\subsection{Hyperboloidal slicing framework: the energy scalar product choice}

Under the light of the previous discussion, the choice of scalar product becomes fundamental for the physical assessment of QNM stability. Following the proposal in [53], we propose a scalar product based on the energy of the propagating field, as the proper measure of big and small in physical scenarios involving QNM perturbations.

In this section we revisit and extend the discussion in [53], to place it on a sounder spacetime ground. We first discuss and motivate such choice of scalar product in our problem. Then we derive the relation between the physical energy norm associated to a scalar field propagating on a spherically symmetric spacetime background and the "effective" stress energy energy used in [53] to define energy and an "effective" energy scalar product. At the end of this section the energy flux at the boundary is obtained.

3.2.1. Elements of the hyperboloidal framework in spherical symmetry. Let $\left(\mathcal{M}, g_{a b}, \nabla\right)$ denote a manifold equipped with is a Lorentzian metric $g$ and associated Levi-Civita connection $\nabla$. Consider a complex scalar field $\Phi$ satisfying the wave equation

$$
\square_{g} \Phi=g^{a b} \nabla_{a} \nabla_{b} \Phi=0 .
$$

The energy momentum tensor associated to this wave equation is given by

$$
T_{a b}=\frac{1}{2}\left(\nabla_{a} \bar{\Phi} \nabla_{b} \Phi-\frac{1}{2} g_{a b} g^{c d} \nabla_{c} \bar{\Phi} \nabla_{d} \Phi+c . c .\right)
$$


This definition can be motivated from stress-enegy conservation, namely by the fact that

$$
g^{a c} \nabla_{c} T_{a b}=\square \Phi \nabla_{b} \bar{\Phi}+\text { c.c. . }
$$

Thus

$$
\square_{g} \Phi=0 \Longrightarrow g^{a c} \nabla_{c} T_{a b}=0 .
$$

The expression (48) corresponds to the energy momentum associated to the action

$$
S=\int_{\mathcal{M}} g^{a b} \nabla_{a} \Phi \nabla_{b} \bar{\Phi} d V_{g}
$$

where $d V_{g}$ is the volume element in $\left(\mathcal{M}, g_{a b}, \nabla\right)$. To motivate the energy scalar product, to be discussed in this section for the QNM problem, we use the notation of the vector field method to put this discussion in a wider context. An introductory discussion of the vector field method can be found in [100]. Let $X^{a}$ be any vector field and let the associated deformation tensor ${ }^{[X]} \pi_{a b}$, the vector and scalar currents, denoted as ${ }^{[X]} J_{a}$ and $K^{[X]}$ respectively, be defined by

$$
{ }^{[X]} J_{a}=T_{a b} X^{b}, \quad{ }^{[X]} \pi_{a b}=\nabla_{(a} X_{b)}, \quad K^{[X]}=T_{a b} \pi^{a b} .
$$

A straightforward calculation shows that

$$
\nabla^{a[X]} J_{a}={ }^{[X]} K
$$

Integration over a spacetime region $\mathcal{R}$ with boundary $\partial \mathcal{R}$ gives the identity

$$
\int_{\partial \mathcal{R}}{ }^{[X]} J_{a} n^{a} d S=\int_{\mathcal{R}}{ }^{[X]} K d V
$$

where $n^{a}$ is the normal to $\partial \mathcal{R}$ and $d \Sigma$ and $d V$ are the associated volume elements in $\partial \mathcal{R}$ and $\mathcal{R}$ respectively. Thus, if $\left(\mathcal{M}, g_{a b}, \nabla\right)$ admits a timelike Killing vector $t^{a}$ then, its associated deformation tensor vanishes and hence, motivates the definition

$$
{ }^{[t]} E=\int_{\Sigma}{ }^{[t]} J_{a} n^{a} d \Sigma
$$

where $\Sigma$ is a spacelike hypersurface with normal $n^{a}$, recovering the expression in [109] for stationary spacetimes. Observe that, in general ${ }^{[t]} E$ is not conserved quantity - see [23] for a comprehensive discussion on the vector field method and the construction of energy estimates.

Aiming at revisiting the discussion of the scalar product in [53], and focusing on the scalar case, we consider the wave equation 47) on a spherically symmetric spacetime $\left(\mathcal{M}, g_{a b}, \nabla\right)$. In particular, we adopt local coordinates $x^{\mu}=\{t, r, \theta, \phi\}$ in which the line element $d s^{2}=g_{\mu \nu} d x^{\mu} d x^{\nu}$ reads

$$
d s^{2}=-f(r) d t^{2}+f(r)^{-1} d r^{2}+r^{2} \Omega_{A B} d x^{A} d x^{B},
$$

where $\Omega_{a b}$ is the standard metric on $\mathbb{S}^{2}$ with coordinates $x^{A}=\{\theta, \phi\}$. By considering the following Ansatz for the field

$$
\Phi=\frac{1}{r} \sum_{\ell m} \phi_{\ell m}(t, r) Y_{\ell m}\left(x^{A}\right),
$$


where $\sum_{\ell m}$ is a shorthand for $\sum_{\ell=0}^{\infty} \sum_{m=-\ell}^{l}$, the wave equation (47) reduces to the mode analysis of

$$
\left(\frac{\partial^{2}}{\partial t^{2}}-\frac{\partial^{2}}{\partial r_{\star}^{2}}+V_{\ell}\right) \phi_{\ell m}=0,
$$

where $r_{\star}$ is a tortoise coordinate defined by $\frac{d r}{d r_{\star}}=f(r)$ and $V_{\ell}=V_{\ell}(r)$. In other words, the spherically symmetric problem is reduced (for each $\ell m$ ) to the analysis of a (1+1)-dimensional wave equation with an effective potential $V$ in Minkowski spacetime $\left(\mathcal{M}, \stackrel{\circ}{\eta}_{i j}, \stackrel{\circ}{\nabla}\right)$,

$$
\square_{\eta} \phi-V \phi=0 .
$$

This equation has an associated effective energy momentum tensor given by

$$
\stackrel{\circ}{T}_{a b}=\frac{1}{2}\left(\stackrel{\circ}{\nabla}_{a} \bar{\phi} \stackrel{\circ}{\nabla}_{b} \phi-\frac{1}{2} \stackrel{\circ}{\eta}_{a b}\left(\stackrel{\circ}{\eta}^{c d} \stackrel{\circ}{\nabla}_{c} \bar{\phi} \stackrel{\circ}{\nabla}_{d} \phi+V \phi \bar{\phi}\right)+c . c .\right),
$$

(when discussing the effective approach the mode indices $\ell m$ are omitted). From this equation, and given a spacelike hypersurface $\Sigma$ and a static Killing vector, one can derive an energy associated to the field $\phi$. This was the strategy followed in [53]. The main purpose of this section is to clarify the relation between the energy associated to the field $\Phi$ and that of the field modes $\phi$ in a hyperboloidal foliation of the spacetime.

Following [53], let us introduce coordinates $(\tau, x)$ adapted to the compactified hyperboloidal foliation via

$$
t=\tau-h(x), \quad r_{\star}=g(x) .
$$

Here, in order to simplify the notation, we have absorbed the length scalar $\lambda$ discussed in [53] into the coordinates $\left(t, r_{\star}\right)$. If one needs to bring the length scalar back, one simply has to identify the coordinates $\left(t, r_{\star}\right)$ with the dimensionless coordinates $(\bar{t}, \bar{x})$ in the discussion of [53]. The function $h(x)$ is known as the height function while the function $g(x)$ is usually chosen to order to compactify the radial coordinate, hence bringing $\mathscr{I}^{+}$to a finite coordinate distance. The line element 56 in these coordinate reads

$$
d s^{2}=f(r) \stackrel{\circ}{i j}_{i j} d x^{i} d x^{j}+r^{2} \Omega_{A B} d x^{A} d x^{B},
$$

with $x^{i}=\{\tau, x\}$ and the 2-dimensional line element,

$$
\stackrel{\circ}{\eta}_{i j} d x^{i} d x^{j}=-d \tau^{2}+\left(\left(g^{\prime}\right)^{2}-\left(h^{\prime}\right)^{2}\right) d x^{2}+2 h^{\prime} d \tau d x .
$$

where we have used ${ }^{\prime}$ to denote a derivative respect to $x$. Let $\Sigma_{\tau}$ be given by,

$$
\Sigma_{\tau}=\left\{x^{\mu} \in \tilde{\mathcal{M}} \mid \tau=0, x \in[a, b], x^{A} \in \mathbb{S}^{2}\right\},
$$

where $\left(\tilde{\mathcal{M}}, \tilde{g}_{a b}\right)$ is the conformal extension of the physical spacetime $\left(\mathcal{M}, g_{a b}\right)$. In contrast with other approaches to include null infinity — for instance that of the conformal Einstein field equations introduced by $\mathrm{H}$. Friedrich; see [32, 108] - in the present hyperboloidal approach, the conformal factor $\Xi$, relating the metrics $\tilde{g}_{a b}=\Xi^{2} g_{a b}$ is not a dynamical quantity but it is fixed a priori and its specific form depends on the choice of hyperboloidal coordinates - see [106, 84, 85]. The function $g(x)$ implements the mapping from ] $-\infty, \infty[$ to $] a, b[$, that is then compactified to $[a, b]$. Then, $a$ and $b$ correspond to the location of the $\mathrm{BH}$ horizon and future null infinity (notice that, depending on the actual implementation, a reflection can happen, so $a$ is actually null infinity, whereas $b$ corresponds to the BH horizon; this is indeed the case for the Schwarzschild implementation in [3, 85, 53]). 
3.2.2. Total energy versus effective mode energy. We can now relate the proper energy of the full spacetime field $\Phi$ with that of the modes $\phi$, namely the one employed in [53] to define the energy norm. A direct calculation shows that the timelike unit normal $n^{a}$ to $\Sigma_{\tau}$ is given by

$$
n^{a}=\frac{1}{\sqrt{f} \sqrt{{g^{\prime 2}}^{2}-{h^{\prime}}^{2}}}\left(w\left(\partial_{\tau}\right)^{a}-\gamma\left(\partial_{x}\right)^{a}\right),
$$

and the volume element is given by

$$
d \Sigma_{\tau}=r^{2} \sqrt{f} \sqrt{g^{\prime 2}-h^{\prime 2}} d x d \Omega
$$

where $d \Omega=\sqrt{|\Omega|} d \theta d \phi$ with $|\Omega|=\operatorname{det}\left[\Omega_{A B}\right]$, is the standard area element on $\mathbb{S}^{2}$, and we have introduced the following notation [53]

$$
w=\frac{g^{\prime 2}-h^{\prime 2}}{g^{\prime}}, \quad \gamma=\frac{h^{\prime}}{g^{\prime}}, \quad p=\frac{1}{g^{\prime}} .
$$

Since we are working on the 4-dimensional spacetime $\left(\mathcal{M}, g_{a b}\right)$ instead of the effective 2-dimensional Minkowski spacetime $\left(\stackrel{\mathcal{M}}{\eta^{\prime}} \stackrel{\circ}{a b}\right)$, the expressions for normal vector $n^{a}$ and volume element $d \Sigma_{\tau}$ reported here and those given in [53] differ by a factor of $\sqrt{f}$. Observe that $\partial_{t}=\partial_{\tau}$ and hence $\partial_{\tau}$ is a Killing vector. Thus, ${ }^{[\tau]} J_{a}$ is divergence free, $\nabla^{a}[\tau] J_{a}=0$, and we have the associated energy associated with (the full) $\Phi$

$$
{ }^{[\tau]} E=\int_{\Sigma_{\tau}}{ }^{[\tau]} J_{a}[\Phi] n^{a} d \Sigma_{\tau}
$$

A direct calculation using the energy momentum tensor $T_{a b}$ as given in equation (48) renders

$$
\begin{aligned}
{ }^{[\tau]} J_{a}[\Phi] n^{a} & =\frac{1}{\sqrt{f} \sqrt{g^{\prime 2}-{h^{\prime 2}}^{2}}}\left(w T_{\tau \tau}-\gamma T_{\tau x}\right) \\
& =\frac{1}{2 \sqrt{f} \sqrt{{g^{\prime 2}-h^{\prime 2}}^{2}}}\left(\frac{g^{\prime 2}-{h^{\prime}}^{2}}{g^{\prime}} \partial_{\tau} \Phi \partial_{\tau} \bar{\Phi}+\frac{1}{g^{\prime}} \partial_{x} \Phi \partial_{x} \bar{\Phi}+\frac{g^{\prime} f}{r^{2}} \Omega_{A B} \partial_{A} \Phi \partial_{B} \bar{\Phi}\right) .
\end{aligned}
$$

Notice in particular that the factor $\sqrt{f} \sqrt{{g^{\prime}}^{2}-h^{\prime 2}}$ coming from the normal $n^{a}$ will combine well with that of the volume element $d \Sigma_{\tau}$ when computing the energy (68). Substituting equations 69, and 66) into 68, gives

$$
{ }^{[\tau]} E=\frac{1}{2} \int_{a}^{b} \int_{\mathbb{S}^{2}} r^{2}\left(w \partial_{\tau} \Phi \partial_{\tau} \bar{\Phi}+p \partial_{x} \Phi \partial_{x} \bar{\Phi}+\frac{g^{\prime} f}{r^{2}} \Omega^{A B} \partial_{A} \Phi \partial_{B} \bar{\Phi}\right) d x d \Omega
$$

Before substituting the Ansatz (57) into equation (70), observe that, using the chain rule, the coordinate transformation $(61)$ and $\partial_{r_{\star}}=f \partial_{r}$, one has

$$
\partial_{x}=-h^{\prime} \partial_{t}+f g^{\prime} \partial_{r}
$$

Then, using the last expression and the Ansatz (57) gives

$$
\partial_{x} \Phi=\sum_{\ell m} \frac{Y_{\ell m}}{r}\left(\partial_{x} \phi_{\ell m}-\frac{f g^{\prime}}{r} \phi_{\ell m}\right) .
$$


Substituting the Ansatz (57) aided by equation (72) into equation (70), renders

$$
\begin{aligned}
{ }^{[\tau]} E=\sum_{\ell m, \ell^{\prime}, m^{\prime}} & \frac{1}{2} \int_{a}^{b} \int_{\mathbb{S}^{2}}\left\{Y _ { \ell m } \overline { Y } _ { \ell ^ { \prime } , m ^ { \prime } } \left(w \partial_{\tau} \phi_{\ell m} \partial_{\tau} \bar{\phi}_{\ell^{\prime} m^{\prime}}+p\left(\partial_{x} \phi_{\ell m}-\frac{f g^{\prime}}{r} \phi_{\ell m}\right)\right.\right. \\
\times & \left.\left.\left(\partial_{x} \bar{\phi}_{\ell^{\prime} m^{\prime}}-\frac{f g^{\prime}}{r} \bar{\phi}_{\ell^{\prime} m^{\prime}}\right)\right)+\frac{g^{\prime} f}{r^{2}} \phi_{\ell m} \bar{\phi}_{\ell^{\prime} m^{\prime}} \Omega^{A B} \partial_{A} Y_{\ell m} \partial_{B} \bar{Y}_{\ell^{\prime} m^{\prime}}\right\} d x d \Omega
\end{aligned}
$$

where $\sum_{\ell m, \ell^{\prime} m^{\prime}}$ is a shorthand for $\sum_{\ell m} \sum_{\ell^{\prime} m^{\prime}}$. Expanding and rearranging gives

$$
{ }^{[\tau]} E=\sum_{\ell m, \ell^{\prime} m^{\prime}} \frac{1}{2} \int_{a}^{b} \int_{\mathbb{S}^{2}}\left\{Y_{\ell m} \bar{Y}_{\ell^{\prime} m^{\prime}}\left(A_{\ell m, \ell^{\prime} m^{\prime}}+B_{\ell m, \ell^{\prime} m^{\prime}}\right)+D_{\ell m, \ell^{\prime} m^{\prime}}\right\} d x d \Omega
$$

with

$$
\begin{aligned}
A_{\ell m, \ell^{\prime} m^{\prime}} & =w \partial_{\tau} \phi_{\ell m} \partial_{\tau} \bar{\phi}_{\ell^{\prime} m^{\prime}}+p \partial_{x} \phi_{\ell m} \partial_{x} \bar{\phi}_{\ell^{\prime} m^{\prime}}+\frac{f g^{\prime}}{r^{2}} \phi_{\ell m} \bar{\phi}_{\ell^{\prime} m^{\prime}} \\
B_{\ell m, \ell^{\prime} m^{\prime}} & =-\frac{f}{r}\left(\bar{\phi}_{\ell^{\prime} m^{\prime}} \partial_{x} \phi_{\ell m}+\phi_{\ell m} \partial_{x} \bar{\phi}_{\ell^{\prime} m^{\prime}}\right) \\
D_{\ell m, \ell^{\prime} m^{\prime}} & =\frac{f g^{\prime}}{r^{2}} \phi_{\ell m} \bar{\phi}_{\ell^{\prime} m^{\prime}} \Omega^{A B} \partial_{A} Y_{\ell m} \partial_{B} \bar{Y}_{\ell^{\prime} m^{\prime}},
\end{aligned}
$$

where we have used that $p=1 / g^{\prime}$ to simplify the expressions. The contribution from the $A_{\ell m, \ell^{\prime} m^{\prime}}$ part can be straightforwardly computed exploiting the orthogonality relation

$$
\int_{\mathbb{S}^{2}} Y_{\ell m} \bar{Y}_{\ell^{\prime} m^{\prime}} d \Omega=\delta_{\ell \ell^{\prime}} \delta_{m m^{\prime}}
$$

as follows

$$
\begin{gathered}
\sum_{\ell m, \ell^{\prime} m^{\prime}} \int_{a}^{b} \int_{\mathbb{S}^{2}} Y_{\ell m} \bar{Y}_{\ell^{\prime}, m^{\prime}} A_{\ell m, \ell^{\prime} m^{\prime}} d x d \Omega= \\
\sum_{\ell m} \int_{a}^{b} w \partial_{\tau} \phi_{\ell m} \partial_{\tau} \bar{\phi}_{\ell m}+p \partial_{x} \phi_{\ell m} \partial_{x} \bar{\phi}_{\ell m}+\frac{f g^{\prime}}{r^{2}} \phi_{\ell m} \bar{\phi}_{\ell m} d x .
\end{gathered}
$$

For determining the contribution of the $D_{\ell m \ell^{\prime} m^{\prime}}$ term, albeit, very cumbersome, one could opt to compute $\partial_{A} Y_{\ell m}$ in a particular coordinate system $x^{A}$ for $\mathbb{S}^{2}$. Instead, it is more convenient to use that

$$
\frac{1}{\sqrt{|\Omega|}} \partial_{A}\left(\sqrt{|\Omega|} \Omega^{A B} \bar{Y}_{\ell^{\prime} m^{\prime}} \partial_{B} Y_{\ell m}\right)=-\ell(\ell+1) \bar{Y}_{\ell^{\prime} m^{\prime}}+\Omega^{A B} \partial_{A} \bar{Y}_{\ell^{\prime} m^{\prime}} \partial_{B} Y_{\ell m}
$$

which follows by using the Leibniz rule and that $\Delta_{\mathbb{S}^{2}} Y_{\ell m}=-\ell(\ell+1) Y_{\ell m}$ where $\Delta_{\mathbb{S}^{2}}$ is the standard ("round") Laplacian on $\mathbb{S}^{2}$. Then integration over $\mathbb{S}^{2}$ and an application of Stokes theorem renders the following expression

$$
\int_{\mathbb{S}^{2}} \Omega^{A B} \partial_{A} Y_{\ell m} \partial_{B} \bar{Y}_{\ell^{\prime} m^{\prime}} d \Omega=\ell(\ell+1) \delta_{\ell \ell^{\prime}} \delta_{m m^{\prime}}
$$

Using the identities 75 and 78 one can easily read the contributions $D_{\ell m \ell^{\prime} m^{\prime}}$ as follows

$$
\sum_{\ell m, \ell^{\prime} m^{\prime}} \int_{a}^{b} \int_{\mathbb{S}^{2}} Y_{\ell m} \bar{Y}_{\ell^{\prime} m^{\prime}} D_{\ell m, \ell^{\prime} m^{\prime}} d x d \Omega=\sum_{\ell m} \int_{a}^{b} \frac{\ell(\ell+1)}{r^{2}} f g^{\prime} \phi_{\ell m} \bar{\phi}_{\ell m} d x .
$$


To compute the contribution from the $B_{\ell m \ell^{\prime} m^{\prime}}$ term we integrate by parts once to obtain

$$
\sum_{\ell m, \ell^{\prime}, m^{\prime}} \int_{a}^{b} \int_{\mathbb{S}^{2}} Y_{\ell m} \bar{Y}_{\ell^{\prime}, m^{\prime}} B_{\ell m, \ell^{\prime} m^{\prime}} d x d \Omega=\sum_{\ell m} \int_{a}^{b} \phi_{\ell m} \bar{\phi}_{\ell m} \partial_{x}\left(\frac{f}{r}\right) d x-\left.\left(\frac{f}{r} \phi_{\ell m} \bar{\phi}_{\ell m}\right)\right|_{a} ^{b} .
$$

Using equation (71) we can rewrite the second term in 80 in a more convenient form for the eventual reconstruction of the effective potential inside the final energy expression,

$$
\partial_{x}\left(\frac{f}{r}\right)=-\frac{f^{2} g^{\prime}}{r^{2}}+\frac{f g^{\prime}}{r} \partial_{r} f .
$$

Altogether, substituting equations (71), (80), (79) and (76) into equation (73) gives

$$
{ }^{[\tau]} E=\sum_{\ell m}^{[\tau]} \stackrel{\circ}{E}_{\ell m}-\left.\left(\frac{f}{2 r} \phi_{\ell m} \bar{\phi}_{\ell m}\right)\right|_{a} ^{b}
$$

with,

$$
{ }^{[\tau]}{\stackrel{\circ}{E_{\ell m}}}=\frac{1}{2} \int_{a}^{b} w \partial_{\tau} \phi_{\ell m} \partial_{\tau} \bar{\phi}_{\ell m}+p \partial_{x} \phi_{\ell m} \partial_{x} \bar{\phi}_{\ell m}+\tilde{V} \phi_{\ell m} \bar{\phi}_{\ell m} d x
$$

where the potential $\tilde{V}$ is given by

$$
\tilde{V}=g^{\prime} V, \quad \text { with } \quad V=f\left(\frac{\ell(\ell+1)}{r^{2}}+\partial_{r} f\right) .
$$

The boundary term in equation 82 can be discarded if one assumes that $f(a)=0$ and $\lim _{x \rightarrow b} \frac{f|\phi|^{2}}{r}=0$ as it is in the Schwarzschild metric case where $a$ and $b$ correspond to the location of the horizon and future null infinity $\|$ The mode-energy ${ }^{[\tau]} E_{\ell m}$ coincides with that used in [53] to compute the pseudospectrum, so the energy norm employed in [53] is correct.

3.2.3. Energy flux at spacetime boundaries. Now that the relation between the physical energy ${ }^{[\tau]} E$ and the effective energy ${ }^{[\tau]} E_{\ell m}$ has been clarified, we employ the effective energy momentum tensor of equation (60) to identify the energy flux at the boundary. We drop again momentarily the mode indices $\ell m$, while discussing the effective approach. First observe that the divergence of the effective energy momentum tensor is given by

$$
\stackrel{\circ}{\nabla}^{a} \stackrel{\circ}{T}_{a b}=-\frac{1}{4} \bar{\phi} \phi \stackrel{\circ}{\nabla}_{b} V+\frac{1}{2}\left(\square_{\eta} \phi-V \phi\right)+c . c .
$$

Notice that, within the effective approach, even if the reduced wave equation (59) is satisfied, $\stackrel{\circ}{\nabla}^{a} \stackrel{\circ}{T}_{a b}=0$ does not hold in general for a non-constant potential. Contrast this situation with the 4-dimensional case where the wave equation (47) implies that $\nabla^{a} T_{a b}=0$. Nonetheless, one can still construct a conserved current in the effective approach since

$$
\stackrel{\circ}{\nabla}_{a}^{[t]} \stackrel{\circ}{J}^{a}=-\frac{1}{2} \phi \bar{\phi} \partial_{t} V .
$$

$\|$ Actually, $a$ and $b$ are interchanged in [3, 85, 53], with $a$ corresponding to null infinity and $b$ to th BH horizon. In more general spacetimes $f$ will vanish on horizons, as it is the case for asymptotically de Sitter spacetimes. 
Hence, using that $\partial_{t} V=0$ one has that ${ }^{[t]} \stackrel{\circ}{J}^{a}$ is a conserved current. Notice that we have added a ring over the current letter to stress the difference between the 4-dimensional associated current ${ }^{[t]} J^{a}=\left(\partial_{t}\right)^{a} T_{a b}$ and the effective 2-dimensional one ${ }^{[t]} \stackrel{\circ}{J}^{a}=\left(\partial_{t}\right)^{a} \stackrel{\circ}{T}_{a b}$. Moreover, recalling that $\partial_{t}=\partial_{\tau}$ one has

$$
[t] \stackrel{\circ}{J}^{a}=[\tau] \stackrel{\circ}{J^{a}} .
$$

Furthermore, using that $\partial_{\tau} V=0$ then

$$
\stackrel{\circ}{\nabla}_{a}^{[\tau]} \stackrel{\circ}{J}^{a}=0 .
$$

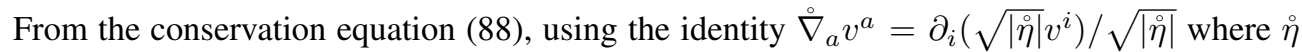
is the determinant of $\stackrel{\circ}{\eta}_{i j}$ in hyperboloidal coordinates $x^{i}=\{\tau, x\}$, a direct calculation shows

$$
\partial_{\tau} \stackrel{\circ}{\mathcal{E}}=\partial_{x} \stackrel{\circ}{\mathcal{F}}
$$

where

$$
\stackrel{\circ}{\mathcal{E}}=\frac{1}{2}\left(w \partial_{\tau} \phi \partial_{\tau} \bar{\phi}+p \partial_{x} \phi \partial_{x} \bar{\phi}+\tilde{V} \phi \bar{\phi}\right)
$$

and the effective flux $\stackrel{\circ}{\mathcal{F}}$ is given by

$$
\stackrel{\circ}{\mathcal{F}}=\gamma \partial_{\tau} \phi \partial_{\tau} \bar{\phi}+p \operatorname{Re}\left(\partial_{\tau} \phi \partial_{x} \bar{\phi}\right) \text {. }
$$

In fact, an alternative - albeit longer - way to derive equation 89 is to apply directly $\partial_{\tau}$ to equation (83) and then use the effective wave equation (59) written in hyperboloidal coordinates to substitute the term $\partial_{\tau}^{2} \phi-$ see [53] for the explicit form of equation [59] in hyperboloidal coordinates. Then, integration by parts leads to an integrated version of the expression for the effective flux $\stackrel{\circ}{\mathcal{F}}$.

Restoring the mode-indices $\ell m$ in equation (89), (90) and (91) to make contact with the total physical energy ${ }^{[\tau]} E$ - as defined in equation 68 - one notices that in fact $\stackrel{\circ}{\mathcal{E}}_{\ell m}=\frac{d}{d x}\left({ }^{[\tau]} \stackrel{\circ}{E}_{\ell m}\right)$, where ${ }^{[\tau]} \stackrel{\circ}{E}_{\ell m}$ is the effective energy given in equation $[83)$. To identify the total physical flux, observe that equation (82) can be re-expressed as

$$
{ }^{[\tau]} E=\sum_{\ell m} \int_{a}^{b}\left(\stackrel{\circ}{\mathcal{E}}_{\ell m}-\partial_{x}\left(\frac{f}{2 r} \phi_{\ell m} \bar{\phi}_{\ell m}\right)\right) d x .
$$

Then applying $\partial_{\tau}$ to the above equation gives

$$
\partial_{\tau}\left({ }^{[\tau]} E\right)=\sum_{\ell m} \int_{a}^{b}\left(\partial_{\tau} \stackrel{\circ}{\mathcal{E}}_{\ell m}-\partial_{x} \partial_{\tau}\left(\frac{f}{2 r} \phi_{\ell m} \bar{\phi}_{\ell m}\right)\right) d x
$$

Using the effective conservation equation (89) and formally integrating in $x$, we get

$$
\partial_{\tau}\left({ }^{[\tau]} E\right)=\left.\sum_{\ell m}\left(\stackrel{\circ}{\mathcal{F}}_{\ell m}-\frac{f}{2 r} \partial_{\tau}\left(\phi_{\ell m} \bar{\phi}_{\ell m}\right)\right)\right|_{a} ^{b} .
$$

Thus, the total flux $F$ satisfying

$$
\partial_{\tau}\left({ }^{[\tau]} E\right)=\left.F\right|_{a} ^{b}
$$


is given explicitly by

$$
F=\sum_{\ell m} \gamma \partial_{\tau} \phi_{\ell m} \partial_{\tau} \bar{\phi}_{\ell m}+p \operatorname{Re}\left(\partial_{\tau} \phi_{\ell m} \partial_{x} \bar{\phi}_{\ell m}\right)-\frac{f}{2 r} \partial_{\tau}\left(\phi_{\ell m} \bar{\phi}_{\ell m}\right) .
$$

For asymptotically flat spacetimes (or for spacetimes regions bounded by horizons, as de Sitter) the $\frac{f}{2 r}$ term vanishes at the boundaries. On the other hand, the function $p$ in the hyperboloidal foliation in [53] is devised also to vanish at the boundaries. This is in particular the case of Schwarzschild spacetime in [53] or Reissner-Nordström in [26]. Hence, the flux expression reduces to

$$
F=\sum_{\ell m} \gamma \partial_{\tau} \phi_{\ell m} \partial_{\tau} \bar{\phi}_{\ell m}
$$

This corresponds to equation (10), in section 2 where our main results have been summarized.

\section{Scalar product in the hyperboloidal approach to QNMs: some applications}

Once the main point of this article is made, namely that the choice of scalar product is crucial for the physical assessment of QNM instability and that the energy scalar product is our proposal for such assessment, in this section we present several applications in which the notion of scalar product relevant for the hyperboloidal approach to the QNM problem.

\subsection{Energy scalar product and QNM weak formulation}

QNMs in the present hyperboloidal setting arise by considering the wave equation 58 in a hyperboloidal slicing and then solving the eigenvalue problem obtained by Fourier transform respect in the time coordinate. Specifically, introducing a first-order reduction in time by $\psi_{\ell m}=\partial_{t} \phi_{\ell m}=\partial_{\tau} \phi_{\ell m}$, and taking the Fourier transform with respect to $\tau$-we simplify the notation by denoting the Fourier transforms of $\phi_{\ell m}$ and $\psi_{\ell m}$ simply by $\phi$ and $\psi$ - we are left with the following eigenvalue problem

$$
L u=\omega u
$$

where $u$ and $L$ are a vector valued function and a differential operator given respectively by

$$
u=\left(\begin{array}{c}
\phi \\
\psi
\end{array}\right), \quad L=\frac{1}{i}\left(\begin{array}{c|c}
0 & 1 \\
\hline L_{1} & L_{2}
\end{array}\right)
$$

with

$$
L_{1}=w^{-1}(x)\left(\partial_{x}\left(p(x) \partial_{x}\right)-q(x)\right) \quad, \quad L_{2}=w^{-1}(x)\left(\gamma(x) \partial_{x}+\partial_{x}(\gamma(x) \cdot)\right),
$$

where $L_{1}$ is a Sturm-Liouville operator, $p, w$ are functions defined in equations 67 and $q=\tilde{V}$ with $\tilde{V}$ as defined in equation (84). These are essentially equations (4), (2) and (3) in section 2.1. The relabeling of the potential $\tilde{V}$ with $q$ is merely aesthetic as it is intended to express the operator $L_{1}$ with standard Sturm-Liouville notation. For further details on the derivation of this eigenvalue problem, see [53]. Let the scalar product inherited from the effective energy ${ }^{[\tau]} E_{\ell m}$ be denoted by $\langle\cdot, \cdot\rangle_{E}$. From equation 83 one has that

$$
\left\langle u_{1}, u_{2}\right\rangle_{E}=\frac{1}{2} \int_{a}^{b}\left(w(x) \bar{\psi}_{1} \psi_{2}+p(x) \partial_{x} \bar{\phi}_{1} \partial_{x} \phi_{2}+\tilde{V}(x) \bar{\phi}_{1} \phi_{2}\right) d x
$$


4.1.1. Weak formulation of the QNM problem. The weak problem is obtained by taking a vector valued test function

$$
u_{T}=\left(\begin{array}{c}
\phi_{T} \\
\psi_{T}
\end{array}\right)
$$

and considering

$$
\left\langle u_{T}, L u\right\rangle_{E}=\omega\left\langle u_{T}, u\right\rangle_{E} .
$$

In a more explicit notation, the above equation reads

$$
\left\langle\left(\begin{array}{c}
\phi_{T} \\
\psi_{T}
\end{array}\right),\left(\begin{array}{c}
\psi \\
L_{1} \phi+L_{2} \psi
\end{array}\right)\right\rangle_{E}=i \omega\left\langle\left(\begin{array}{c}
\phi_{T} \\
\psi_{T}
\end{array}\right),\left(\begin{array}{c}
\phi \\
\psi
\end{array}\right)\right\rangle_{E} .
$$

Using explicitly the form of $\langle\cdot, \cdot\rangle_{E}$ one has

$$
\begin{aligned}
& \int_{a}^{b}\left[w \bar{\psi}_{T}\left(L_{1} \phi+L_{2} \psi\right)+p \partial_{x} \bar{\phi}_{T} \partial_{x} \psi+\tilde{V} \bar{\phi}_{T} \psi\right] d x \\
&=i \omega \int_{a}^{b}\left[w \bar{\psi}_{T} \psi+p \partial_{x} \bar{\phi}_{T} \partial_{x} \phi+\tilde{V} \bar{\phi}_{T} \phi\right] d x
\end{aligned}
$$

One of the advantages of the weak formulation is that one can reduce the number of derivatives in the equation under the integral sign by integrating by parts. In our case, $L_{1}$ is a second order operator, so it is convenient to do so. Performing the integrating by parts of the " $L_{1}$ part" gives

$$
\begin{aligned}
\int_{a}^{b} w \bar{\psi}_{T} L_{1} \phi d x=\int_{a}^{b} \bar{\psi}_{T} \partial_{x}\left(p \partial_{x} \phi\right) & -q \bar{\psi}_{T} \phi d x \\
& =\left.\left(p \bar{\psi}_{T} \partial_{x} \phi\right)\right|_{a} ^{b}-\int_{a}^{b} p \partial_{x} \phi \partial_{x} \bar{\psi}_{T}+q \bar{\psi}_{T} \phi d x
\end{aligned}
$$

Since $L_{2}$ is a first order operator one can opt to leave either it as it is, or rather integrate it by parts. Notice that this option is not available for the third term $p \partial_{x} \bar{\phi}_{T} \partial_{x} \psi$ in equation 105 since integrating by parts would increase the number of derivatives in the integrand. Integrating the " $L_{2}$ part" by parts renders

$$
\int_{a}^{b} w \bar{\psi}_{T} L_{2} \psi d x=\int_{a}^{b} \bar{\psi}_{T}\left(\gamma \partial_{x} \psi+\partial_{x}(\gamma \psi)\right) d x=\left.2\left(\gamma \bar{\psi}_{T} \psi\right)\right|_{a} ^{b}-\int_{a}^{b} w \psi L_{2} \bar{\psi}_{T} d x
$$

Thus, we have two options for the weak formulation of our QNM eigenvalue problem:

i) Integrating by parts both $L_{1}$ and $L_{2}$. This gives the following weak problem

$$
\int_{a}^{b} W_{L}\left(u_{T}, u\right) d x+\left.W_{B}\left(u_{T}, u\right)\right|_{a} ^{b}=i \omega \int_{a}^{b} W_{R}\left(u_{T}, u\right) d x
$$

where

$$
\begin{aligned}
& W_{L}\left(u_{T}, u\right)=p\left(\partial_{x} \bar{\phi}_{T} \partial_{x} \psi-\partial_{x} \phi \partial_{x} \bar{\psi}_{T}\right)+q\left(\bar{\phi}_{T} \psi-\bar{\psi}_{T} \phi\right)-w \psi L_{2} \bar{\psi}_{T} \\
& W_{R}\left(u_{T}, u\right)=p \partial_{x} \bar{\phi}_{T} \partial_{x} \phi+w \bar{\psi}_{T} \psi+q \bar{\phi}_{T} \phi \\
& W_{B}\left(u_{T}, u\right)=p \bar{\psi}_{T} \partial_{x} \phi+2 \gamma \bar{\psi}_{T} \psi
\end{aligned}
$$


ii) Integrating by parts only of $L_{1}$. If one opts not to integrate by parts the $L_{2}$ term one is left with the following alternative weak problem

$$
\int_{a}^{b} W_{L}^{*}\left(u_{T}, u\right) d x+\left.W_{B}^{*}\left(u_{T}, u\right)\right|_{a} ^{b}=i \omega \int_{a}^{b} W_{R}\left(u_{T}, u\right) d x,
$$

where

$$
\begin{aligned}
& W_{L}^{*}\left(u_{T}, u\right)=p\left(\partial_{x} \bar{\phi}_{T} \partial_{x} \psi-\partial_{x} \phi \partial_{x} \bar{\psi}_{T}\right)+q\left(\bar{\phi}_{T} \psi-\bar{\psi}_{T} \phi\right)+w \bar{\psi}_{T} L_{2} \psi \\
& W_{B}^{*}\left(u_{T}, u\right)=p \bar{\psi}_{T} \partial_{x} \phi
\end{aligned}
$$

4.1.2. Formal adjoint $L^{\dagger}$ in the weak formulation. Equation $(108)$ - or alternatively (110)constitutes the weak formulation of the problem, the main focus of this section. For completeness, notice that this discussion is very close in spirit to the determination of the formal adjoint. Indeed, if we integrate by parts the term $p \partial_{x} \bar{\phi}_{T} \partial_{x} \psi$ and use $q=\tilde{V}$ we obtain

$$
\begin{array}{r}
-\int_{a}^{b}\left[w \psi\left(L_{1} \bar{\phi}_{T}+L_{2} \bar{\psi}_{T}\right)+\partial_{x} \phi \partial_{x} \bar{\psi}_{T}+\tilde{V} \phi \bar{\psi}_{T}\right] d x+\left.\left(p\left(\bar{\psi}_{T} \partial_{x} \phi+\psi \partial_{x} \bar{\phi}_{T}\right)+2 \gamma \bar{\psi}_{T} \psi\right)\right|_{a} ^{b} \\
=i \omega \int_{a}^{b}\left[w \bar{\psi}_{T} \psi+p \partial_{x} \bar{\phi}_{T} \partial_{x} \phi+\tilde{V} \bar{\phi}_{T} \phi\right] d x,
\end{array}
$$

which in turn can be rewritten as

$$
\left\langle\left(\begin{array}{c}
\psi_{T} \\
L_{1} \phi_{T}+L_{2} \psi_{T}
\end{array}\right),\left(\begin{array}{c}
\phi \\
\psi
\end{array}\right)\right\rangle_{E}+B=i \omega\left\langle\left(\begin{array}{c}
\phi_{T} \\
\psi_{T}
\end{array}\right),\left(\begin{array}{c}
\phi \\
\psi
\end{array}\right)\right\rangle_{E},
$$

where

$$
B=\left.\left(p\left(\bar{\psi}_{T} \partial_{x} \phi+\psi \partial_{x} \bar{\phi}_{T}\right)+2 \gamma \bar{\psi}_{T} \psi\right)\right|_{a} ^{b} .
$$

If one assumes that $p(a)=p(b)=0$ - as it is indeed the case in the operator $L$ constructed in the compactified hyperboloidal formulation - then one can absorb the boundary term and identify the formal adjoint of $L$ as

$$
L^{\dagger}=\frac{1}{i}\left(\begin{array}{c|c}
0 & 1 \\
\hline L_{1} & L_{2}+L_{2}^{\partial}
\end{array}\right)
$$

where

$$
L_{2}^{\partial}=2 \frac{\gamma}{w}(\delta(x-a)-\delta(x-b))
$$

which recovers the expression found in [53].

4.1.3. QNM calculation in the weak formulation: finite elements. Equation $(108)$ - or alternatively equation (110) — represents the weak formulation of the eigenvalue problem (98). From a numerical perspective this formulation of the problem naturally opens the possibility to use finite elements methods. This offers an approach to validate the results obtained with Chebyshev spectral methods in [53, 54, 26]. To probe these ideas in a controlled arena, we test the Pöschl-Teller potential case, used as a benchmark in the study of QNMs since it can be solved exactly, yielding to the following expression for the frequencies

$$
\omega_{n}^{ \pm}= \pm \frac{\sqrt{3}}{2}+i\left(n+\frac{1}{2}\right)
$$


For the numerical implementation of finite elements we use a simple automated implementation using the FEniCS software. The Pöschl-Teller potential reads

$$
V\left(r_{\star}\right)=V_{o} \operatorname{sech}^{2}\left(r_{\star}\right), \quad r_{\star} \in(-\infty, \infty) .
$$

where $V_{o}$ is a constant. Using the same hyperboloidal foliation (Bizon-Mach coordinates) as that discussed for the Pöschl-Teller potential in [53], corresponds to the following prescription for the height and spatial compactification functions: $h(x)=\frac{1}{2} \ln \left(1-x^{2}\right)$ and $g(x)=$ $\operatorname{arctanh}(x)$, on the interval $[a, b]=[-1,1]$. The latter, in turn, implies

$$
w(x)=1, \quad p(x)=1-x^{2}, \quad q(x)=\tilde{V}=V_{o}, \quad \gamma(x)=-x .
$$

As anticipated, in particular it holds $p(a)=p(b)=0$ which simplifies the boundary term $W_{B}\left(u, u_{T}\right)$ in equations (108) and (110). If one assumes that the test vector valued function is of "compact support in $[a, b]$ " consistently with the theory of distributions - see [31]then the boundary term vanishes and we are left with the problem

$$
\int_{a}^{b} W_{L}\left(u_{T}, u\right) d x=i \omega \int_{a}^{b} W_{R}\left(u_{T}, u\right) d x
$$

or alternatively

$$
\int_{a}^{b} W_{L}^{*}\left(u_{T}, u\right) d x=i \omega \int_{a}^{b} W_{R}\left(u_{T}, u\right) d x .
$$

A straightforward implementation in FEniCS using the linear algebra PETSc solver renders the numerical results for the first few eigenvalues extracted from the weak problem (119) and 120 as shown in figure 4 and figure 5 respectively.

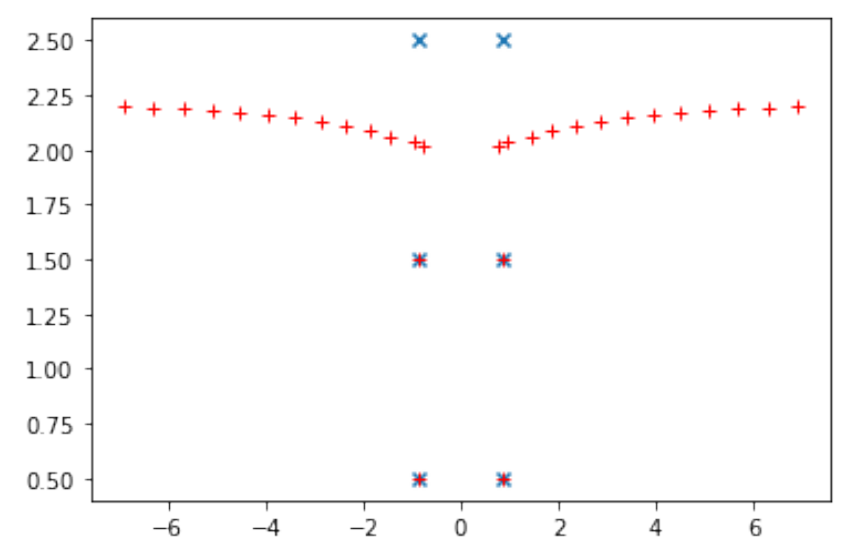

Figure 4. Pöschl-Teller QNM calculation as a variational problem solved in FEniCS with a mesh with $\mathrm{N}=500$ points and using the Lagrange finite elements function space "CG" (Continuous Garlekin). In blue the exact known eigenvalues of the operator $L$ are shown and in red the numerically computed

One can readily observe from Figures 4 and 5 that the first two eigenvalues are recovered and that from the third eigenvalue onward the analytical result and numerical one disagree. Additionally, the weak problem (119) gives direct access to the eigenvalues of $L$ while the weak problem (120) renders the eigenvalues of $L^{\dagger}$. As extensively discussed in [53], in 


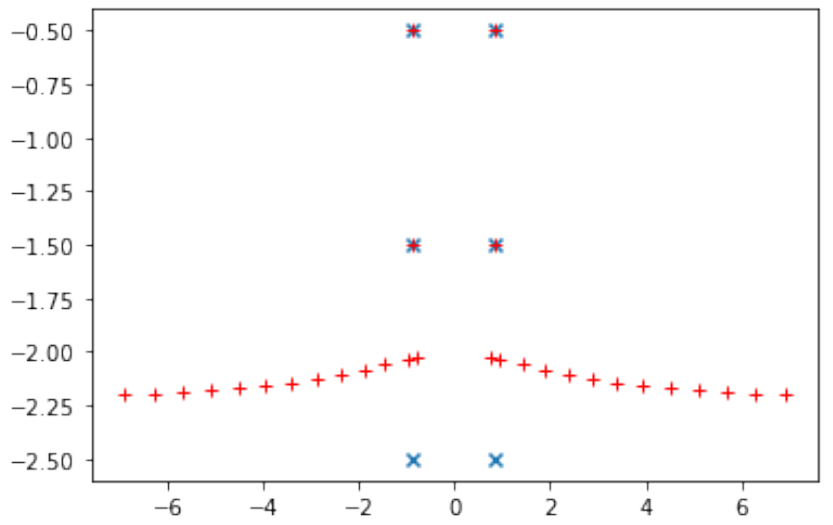

Figure 5. Pöschl-Teller QNM calculation as a variational problem solved in FEniCS with a mesh with $\mathrm{N}=500$ points and using the Lagrange finite elements function space "CG" (Continuous Garlekin). The eigenvalues of $L^{\dagger}$ are $\bar{\omega}$ where $\omega$ are the eigenvalues of $L$. In blue the exact eigenvalues of the $L^{\dagger}$ operator are shown and in red the numerically computed

order to increase the number of correct eigenvalues recovered it is necessary to use enhanced machine precision and the numerical error can be reinterpreted as a perturbation of the operator $L$. The branches where the eigenvalues migrate due to the numerical error are qualitatively similar to those reported in [53] giving confidence that this observed behavior, suitably interpreted as a random perturbation of the $L$ operator, is not exclusive to the spectral approach used in [53]. A full numerical analysis is not pursued here as we consider this discussion as just a proof of concept where the choice of inner product is brought to the forefront of the numerical scheme by considering the weak formulation of the equations.

\subsection{Scalar product and QNM resonant expansions: towards BH spectroscopy}

As a second application of the use of scalar product in the QNM setting we discuss resonant expansions of the propagating field in terms of QNMs. This point has received much attention in the physics literature, going back (at least) to Gamow's description of $\alpha$ decay [36]. In the gravitational setting it is closely related to the discussion of completeness of QNMs of BHs and compact objects (cf. e.g. [20, 81, 12] and references therein). In particular, it is very interesting the cross-fertilization in this field between gravitational and optical studies [70, 19, 66]. In the present section we revisit this discussion with the emphasis on the normalizability of QNMs, consequence of the use of a hyperboloidal foliation. This permits to work on an actual Hilbert space where the scalar product plays a crucial role.

More specifically, our discussion of the expansion of a propagated (scattered) field in QNMs connects more directly with the treatment of this problem in Lax-Phillips scattering theory [67] (see also, for detailed reviews, e.g. [101, 115, 29]). The latter stands as an approach to resonances in terms of poles of the meromorphic extension of the resolvent. As commented above, here we revisit the problem in an approach that uses critically the Hilbert space structure in the hyperbolic approach to resonances, in particular the scalar product we are here discussing. Specifically, in the setting of the non-selfadjoint spectral problem defined in the hyperbolic approach to QNMs, we make use of an asymptotic expansion by Keldysh of the resolvent of a non-selfadjoint operator [59, 60, 78, 13, 14].

This approach allows us to recover the results in Lax-Phillips. But, beyond that and 
in contrast with the standard (Cauchy) approach to resonances, the normalizability of QNM eigenfunctions in the hyperboloidal setting allows us to introduce expansion coefficients, for which we provide explicit expressions that reduce, in the selfadjoint case, to the standard normal modes expressions. This could provide some insight into the quantification of the strength of QNM ringing discussed and related "excitation coefficients" in [81].

4.2.1. Resolvent of non-selfadjoint operators: Keldysh's expansion. Let us consider a nonselfadjoint operator $L$ in a Hilbert (more generally, Banach) space, and its adjoint $L^{\dagger}$ with respect to the given scalar product $\langle\cdot, \cdot\rangle$. Then, as introduced in equation $(12)$, right- $v_{n}$ and left- $w_{n}$ (proper) eigenvectors are defined, respectively, as the eigenvectors of $L$ and $L^{\dagger}$

$$
L v_{n}=\lambda_{n} v_{n}, L^{\dagger} w_{n}=\bar{\lambda}_{n} w_{n}
$$

Notice that $v_{n}$ and $w_{n}$ are normalizable vectors (in contrast to equation (12), we have not yet normalised them). Let us assume, for simplicity, that $L$ and $L^{\dagger}$ are diagonalizable and that their eigenvalues are simple (for the general case, see [78, 13]). In this context, instead of a standard normalization in terms of the norm of individual vectors, let us adopt the condition $\dagger$

$$
\left\langle w_{n}, v_{n}\right\rangle=-1
$$

We can then normalize one of them, but not both. That is, in general $v_{n}$ and $w_{n}$ are not normalized: $\left\|v_{n}\right\| \neq 1 \neq\left\|w_{n}\right\|$. In this setting, we consider $\ddagger$ a bounded domain $\Omega \in \lambda$ - $\mathbb{C}$. Under appropriate hypothesis (namely the discreteness and isolation of $\lambda_{n}$ in the spectrum $\sigma(L)$, guaranteed if $L$ is Fredholm), there is a finite number $N$ of eigenvalues $\lambda_{n} \in \Omega$.

With these elements, we can introduce Keldysh's expansion [59, 60, 78, 13, 14] of the resolvent of $L$, namely $(L-\lambda)^{-1}$, where $\lambda \in \Omega \backslash \sigma(L)$. Specifically, the Green function (the integral Kernel of the resolvent) for $\lambda \in \Omega \backslash \sigma(L)$ can be written as

$$
G_{\lambda}\left(x^{\prime}, x\right)=\sum_{\lambda_{j} \in \Omega} \frac{w_{j}^{\dagger}\left(x^{\prime}\right) v_{j}(x)}{\lambda-\lambda_{j}}+H\left(x, x^{\prime} ; \lambda\right)
$$

where $H\left(x, x^{\prime} ; \lambda\right)$ is analytic in $\Omega$ (see full technical details of this case in [13]). Then, we can formally write the action of the resolvent on a given source $S=S(x)$ (see below in equation (138) in terms of the scalar product, namely taking care of the integration in the $x^{\prime}$ variable

$$
\left((L-\lambda)^{-1} S\right)(\lambda, x)=\left\langle G_{\lambda}(\cdot, x), S\right\rangle+H(\lambda)(S)=\sum_{\lambda_{j} \in \Omega} \frac{v_{j}(x)}{\lambda-\lambda_{j}}\left\langle w_{j}, S\right\rangle+H(\lambda)(S)
$$

where $H(\lambda)$ is an operator, analytic in $\lambda \in \Omega$. This notation is a bit cumbersome and can be made more transparent in the terms of familiar (formal) "bra's"s and "ket's", that take care of the adequate application of the scalar product

$$
(L-\lambda)^{-1}=\sum_{\lambda_{j} \in \Omega} \frac{\left|v_{j}\right\rangle\left\langle w_{j}\right|}{\lambda-\lambda_{j}}+H(\lambda), \lambda \in \Omega \backslash \sigma(L),
$$

$\dagger$ This condition can be generalized, on the one hand, to degenerate eigenvalues and operators with non-trivial Jordan blocks and, on the other hand, to spectral problems with a non-linear dependence on the spectral parameter, namely pencil operators (see [59, $60,78,[3,14]$ ).

$\ddagger$ We use the notation $\lambda$ - $\mathbb{C}$ to refer to the complex plane in the complex variable $\lambda$. This is to distinguish the different spectral parameters appearing in the discussion. 
where $\langle\cdot|$ and $|\cdot\rangle$ must be understood with respect to the given $\langle\cdot, \cdot\rangle$ in the Hilbert space. Let us denote the relation 125 formally as

$$
(L-\lambda)^{-1} \sim \sum_{\lambda_{n} \in \Omega} \frac{\left|v_{n}\right\rangle\left\langle w_{n}\right|}{\lambda-\lambda_{n}}, \lambda \in \Omega \backslash \sigma(L),
$$

where the resolvent in a (bounded) $\Omega$ region is written as a finite sum of poles plus an (omitted) analytical function. Note that the sum is a finite one and not a series. This is a key point for the later interpretation of the asymptotic nature of the resonant expansion, in contrast with convergent series of selfadjoint operators. That is, in spite of the formal similitude to the expression of the resolvent for selfadjoint operators in terms of eigenfunctions and eigenvalues, the "sum" symbol has a completely different content (see [1] for details).

We can use condition $\left\langle w_{n}, v_{n}\right\rangle=-1$ to recast equation 126 as

$$
(L-\lambda)^{-1} \sim \sum_{\lambda_{n} \in \Omega} \frac{\left|v_{n}\right\rangle\left\langle w_{n}\right|}{\left\langle w_{n}, v_{n}\right\rangle} \frac{-1}{\lambda-\lambda_{n}}=\sum_{\lambda_{n} \in \Omega} \frac{\left|v_{n}\right\rangle\left\langle w_{n}\right|}{\left\langle w_{n}, v_{n}\right\rangle} \frac{1}{\lambda_{n}-\lambda} .
$$

Introducing now the condition number $\kappa_{n}$ associated with the eigenvalue $\lambda_{n}$

$$
\kappa_{n}:=\frac{\left\|w_{n}\right\|\left\|v_{n}\right\|}{\left\langle w_{n}, v_{n}\right\rangle}
$$

we can write

$$
(L-\lambda)^{-1} \sim \sum_{\lambda_{n} \in \Omega} \kappa_{n} \frac{\left|v_{n}\right\rangle\left\langle w_{n}\right|}{\left\|w_{n}\right\||| v_{n} \|} \frac{1}{\lambda_{n}-\lambda},
$$

and, in terms of the normalized left- and right-eigenvectors

$$
\hat{w}_{n}=\frac{w_{n}}{\left\|w_{n}\right\|}, \hat{v}_{n}=\frac{v_{n}}{\left\|v_{n}\right\|},
$$

we can finally write (note that expression (15) for $\kappa_{n}$ is here recovered, when using in particular the energy scalar product)

$$
(L-\lambda)^{-1} \sim \sum_{\lambda_{n} \in \Omega} \kappa_{n} \frac{\left|\hat{v}_{n}\right\rangle\left\langle\hat{w}_{n}\right|}{\lambda_{n}-\lambda}, \lambda \in \Omega \backslash \sigma(L) .
$$

Note that this expression formally recovers the expression of the resolvent of a selfadjoint (more generally, 'normal') operator, in which $\hat{w}_{n}=\hat{v}_{n}$ and $\kappa_{n}=1$. However, the statement in the selfadjoint case is much stronger, since the sum there indicates a series convergence. In our case, if $L$ is actually selfadjoint case, expression (131) can indeed be extended to the whole $\lambda$ - $\backslash$ \} \sigma ( L ) \text { in a convergent sense, but this requires other (Hilbert space) techniques. }

4.2.2. QNM resonant expansions in the hyperboloidal approach. Let us apply the previous discussion in the context of the hyperboloidal initial data problem of a wave equation for a field $\phi$ on a domain $\Sigma \times \mathbb{R}^{+}$, formulated in a first-order in time setting and, for concreteness, employing the energy scalar product $\langle\cdot, \cdot\rangle_{E}$. We start, as in equation (1), by reducing the wave equation to a first-order (in time) formulation

$$
\psi=\partial_{\tau} \phi, u=\left(\begin{array}{l}
\phi \\
\psi
\end{array}\right) .
$$


Then, the wave equation can be written as

$$
\left\{\begin{array}{l}
\partial_{\tau} u=i L u \\
u(\tau=0, x)=u_{0}(x),\left\|u_{0}\right\|_{E}<0
\end{array}\right.
$$

where the operator $L$ and its adjoint $L^{\dagger}$ with respect to the scalar product $\langle\cdot, \cdot\rangle_{E}$-we repeat here the equations (2) and (99) for $L$ and equation (114) for $L^{\dagger}$ - have the structure

$$
L=\frac{1}{i}\left(\begin{array}{c|c}
0 & 1 \\
\hline L_{1} & L_{2}
\end{array}\right) \quad, \quad L^{\dagger}=\frac{1}{i}\left(\begin{array}{c|c}
0 & 1 \\
\hline L_{1} & L_{2}+L_{2}^{\partial}
\end{array}\right) .
$$

Explicit expressions for $L_{1}, L_{2}$ and $L_{2}^{\partial}$ in the 1+1-dimensional case are given in equations (100) - see also (3) - and (115).

We address the resolution of the time evolution problem (133) in a spectral setup, adopting a Laplace transform approach. Specifically, we consider for $\operatorname{Re}(s)>0$

$$
u(s ; x):=(\mathcal{L} u)(s ; x)=\int_{0}^{\infty} e^{-s \tau} u(\tau, x) d \tau .
$$

Applying this transformation to equation (133), we get

$$
s u(s ; x)-u(\tau=0, x)=\frac{1}{i} L u(s ; x) .
$$

Dropping the explicit $s$-dependence and using $(133)$ for the initial data, we can write

$$
(L+i s) u(s ; x)=i S(x),
$$

where the source $S$ is defined in terms of the initial data

$$
S(x):=u_{0}(x)
$$

This is a linear non-homogeneous equation. For its resolution in a Green's function approach, we need an expression for the resolvent of $L$. This is the point in which the above-discussed Keldysh's expansion of the resolvent enters. We need then first to consider the eigenfunctions of the homogeneous part of the equation. Writing, for notation purposes, equation (137) in terms of the Fourier parameter $\omega$ by using the relation $s=i \omega$, we can write

$$
(L-\omega) u(\omega ; x)=i S(x) .
$$

The spectral problem of $L$ associated with the homogeneous part is given in (12)

$$
L \hat{v}_{n}=\omega_{n} \hat{v}_{n}, L^{\dagger} \hat{w}_{n}=\bar{\omega}_{n} \hat{w}_{n},
$$

namely the spectral problem (12) in the $\omega$-spectral parameter and for normalized vectors $\hat{v}_{n}$ and $\hat{w}_{n}$ with respect to the energy scalar product $\langle\cdot, \cdot\rangle_{E}$. From this spectral problem, the resolvent $(L-\omega)^{-1}$ is constructed in a bounded $\Omega_{\omega} \in \omega-\mathbb{C} \backslash \sigma(L)$, so $u(\omega ; x)$ writes

$$
u(\omega ; x)=i(L-\omega)^{-1} S, \omega \in \Omega_{\omega} \backslash \sigma(L),
$$

and using equation 131

$$
u(\omega ; x)=i(L-\omega)^{-1} S \sim i \sum_{\omega_{j} \in \Omega_{\omega}} \kappa_{j} \frac{\left\langle\hat{w}_{j} \mid S\right\rangle_{E}}{\omega_{j}-\omega}\left|\hat{v}_{j}\right\rangle .
$$


Writing it again in terms of the Laplace spectral parameter $s=i \omega$, with $s \in \Omega_{s}=i \Omega_{\omega}$ and reinserting the analytic part of the resolvent in 125 , we have, for $s \in \Omega_{s} \backslash \sigma(L)$

$$
u(s ; x)=\sum_{s_{j} \in \Omega_{s}} \kappa_{j} \frac{\left\langle\hat{w}_{j} \mid S\right\rangle_{E}}{s-s_{j}}\left|\hat{v}_{j}\right\rangle+i H(s)(S) .
$$

In order to construct the time evolution solution $u(\tau, x)$ we take the inverse Laplace transform

$$
u(\tau, x)=\frac{1}{2 \pi i} \int_{c-i \infty}^{c+i \infty} e^{s t} u(s ; x) d s
$$

with $c \in \mathbb{R}^{+}$. But expression 143 only provides $u(s ; x)$ in a bounded $\Omega_{s} \backslash \sigma(L)$. In this setting, taking a finite $R \in \mathbb{R}$, we can write (assuming the limit exists)

$$
\begin{aligned}
u(\tau, x) & =\lim _{R \rightarrow \infty} \frac{1}{2 \pi i} \int_{c-i R}^{c+i R} e^{i s} u(s ; x) d s \\
& =\lim _{R \rightarrow \infty} \frac{1}{2 \pi i} \int_{c-i R}^{c+i R} e^{i s}\left(\sum_{s_{j} \in \Omega_{s}} \kappa_{j} \frac{\left\langle\hat{w}_{j} \mid S\right\rangle_{E}}{s-s_{j}}\left|\hat{v}_{j}\right\rangle+i H(s)(S)\right) d s
\end{aligned}
$$

Let us consider now a contour $C$ in $s-\mathbb{C}$ formed by the interval $[c-i R, c+i R]$ closed on the left half-plane by a circle (of radius $R$, centered at $c+i 0$ ) and denote by $\Omega_{R}$ the bounded domain in $s-\mathbb{C}$ delimited by $C$. In the context of the (Fredholm) operators we are considering, the number of $s_{j} \in \Omega_{R}$ is finite, so we can safely interchange the (finite) sum and the integral

$u(\tau, x)=\lim _{R \rightarrow \infty}\left(\sum_{\omega_{j} \in \Omega} \frac{1}{2 \pi i} \oint_{C} e^{s \tau} \kappa_{j} \frac{\left\langle\hat{w}_{j} \mid S\right\rangle_{E}}{s-s_{j}}\left|\hat{v}_{j}\right\rangle d s+\frac{1}{2 \pi} \oint_{C} e^{s \tau} H(s)(S) d s+(\right.$ circle part $\left.)\right)$.

The contour $C$ integral involving the analytic expression $e^{s \tau} H(s)(S)$ vanishes, but nothing guarantees that its integral along the semi-circle vanishes, this depending on the specific dependence of the function $H(s)$, that is not fixed by Keldysh expansion. In general, the last term "(circle part)" gives a term $C_{R}(\tau ; S)$. Using now Cauchy theorem, we can write

$$
u(\tau, x)=\lim _{R \rightarrow \infty}\left(\sum_{\omega_{j} \in \Omega} e^{s_{j} t} \kappa_{j}\left\langle\hat{w}_{j} \mid S\right\rangle_{E} \hat{v}_{j}+C_{R}(\tau ; S)\right) .
$$

In contrast with the selfadjoint case, nothing guarantees that this limits exists. First, for strongly non-selfadjoint (more generally non-normal) operators the condition number $\kappa_{n}$ can present a strong growth and, on the other hand, the terms $C_{R}(\tau ; S)$ does not need to converge as $R \rightarrow \infty$, in particular not needing to vanish. In this context we cannot write $u(\tau, x)$ as an actual convergent series, but we can still write an asymptotic QNM resonant expansion

$$
u(\tau, x) \sim \sum_{n} e^{s_{j} \tau} \kappa_{j}\left\langle\hat{w}_{j} \mid S\right\rangle_{E} \hat{v}_{j}
$$

or, in the Fourier spectral-parameter

$$
u(\tau, x) \sim \sum_{n} e^{i \omega_{j} \tau} \kappa_{j}\left\langle\hat{w}_{j} \mid S\right\rangle_{E} \hat{v}_{j}
$$

this meaning that in a bounded domain $\Omega$ the number of QNMs is finite and we can write

$$
u(\tau, x)=\sum_{\omega_{j} \in \Omega} e^{i \omega_{j} \tau} \kappa_{j}\left\langle\hat{w}_{j} \mid S\right\rangle_{E} \hat{v}_{j}+E_{\Omega}(\tau ; S)
$$


where the structure of the term $e^{s \tau} H(s)(S)$ in the "(circle part)" of 146 permits to bound the "error" $E_{\Omega}(\tau ; S)$ in the estimation of $u(\tau, x)$ by the (finite) resonant expansion. Explicitly, defining $a=\max \{\operatorname{Im}(\omega), \omega \in \Omega\}$, there exists a constant $C_{\Omega}(a, L)$ depending on $a$ and the operator $L$ (but not on the initial data), such that

$$
\left\|E_{\Omega}(\tau ; S)\right\|_{E} \leq C_{\Omega}(a, L) e^{-a \tau}\|S\|_{E} .
$$

On behalf of clarity, denoting by $N$ the (finite) number of QNMs in $\Omega$ and numbering $\omega_{n}$ from 1 to $N$, we can rewrite equation 150 as

$$
\begin{array}{ll}
u(\tau, x)= & \sum_{j=1}^{N} e^{i \omega_{j} \tau} \kappa_{j}\left\langle\hat{w}_{j} \mid S\right\rangle_{E} \hat{v}_{j}+E_{N}(\tau ; S) \\
\text { with }\left.\quad\left\|E_{N}(\tau ; S)\right\|\right|_{E} \leq C_{N}(a, L) e^{-a \tau}\|S\|_{E} .
\end{array}
$$

This is essentially the content of Lax-Phillips resonant expansion [67, 101, 115, 29], here expressed in terms of normalizable QNM functions $\hat{v}_{j}$ and providing an explicit prescription for the evaluation of the associated coefficient. In sum, we can write

$$
u(\tau, x) \sim \sum_{j} e^{i \omega_{j} \tau} a_{j} \hat{v}_{j}(x) \quad, \quad \text { with } a_{j}=\kappa_{j}\left\langle\hat{w}_{j} \mid u_{0}\right\rangle_{E},
$$

where we have rewritten $S$ in terms of initial data $u_{0}$. This provides the QNM resonant expansion of the propagating field in terms of its initial data, in particular entailing a prescription for the quantification of the QNM ringing in the signal [81].

4.2.3. Keldysh QNM expansion for the scattered field. In order to facilitate the comparison of expression (153) with the natural quantities in QNM resonant expansions in (the secondorder formulation of) Lax-Phillips theory, let us rewrite the main steps above explicitly in terms of $\phi$ and $\psi$ fields in equation (132). First, the wave equation (133) writes

$$
\left\{\begin{array}{l}
\partial_{\tau}\left(\begin{array}{l}
\phi \\
\psi
\end{array}\right)=i L\left(\begin{array}{l}
\phi \\
\psi
\end{array}\right) \\
\left(\begin{array}{l}
\phi \\
\psi
\end{array}\right)(t=0, x)=\left(\begin{array}{l}
\varphi_{0}(x) \\
\varphi_{1}(x)
\end{array}\right), \varphi_{0} \in H_{V}^{1}, \varphi_{1} \in L^{2}
\end{array}\right.
$$

where the operator $L$ and its adjoint have the structure in equations (134). Regarding the scalar product, expression (101) is generalised to (odd) dimension $n$ in the form

$$
\left\langle\left(\begin{array}{l}
\phi_{1} \\
\psi_{1}
\end{array}\right),\left(\begin{array}{l}
\phi_{2} \\
\psi_{2}
\end{array}\right)\right\rangle_{E}=\frac{1}{2} \int_{\Sigma}\left(w(x) \overline{\psi_{1}} \psi_{2}+p(x) \overline{\nabla \phi_{1}} \cdot \nabla \phi_{2}+\tilde{V} \overline{\phi_{1}} \phi_{2}\right) d \Sigma,
$$

Under Laplace transform, the source of equation 137 is explicitly written in terms of the initial data as

$$
S=\left(\begin{array}{l}
\varphi_{0} \\
\varphi_{1}
\end{array}\right)
$$

Then, the right and left spectral problem 140 writes, by using

$$
\hat{v}_{n}=\left(\begin{array}{c}
\hat{\phi}_{n}^{\mathrm{R}} \\
\hat{\psi}_{n}^{\mathrm{R}}
\end{array}\right), \hat{w}_{n}=\left(\begin{array}{c}
\hat{\phi}_{n}^{\mathrm{L}} \\
\hat{\psi}_{n}^{\mathrm{L}}
\end{array}\right)
$$

as

$$
\left(\begin{array}{c|c}
0 & 1 \\
\hline L_{1} & L_{2}
\end{array}\right)\left(\begin{array}{c}
\hat{\phi}_{n}^{\mathrm{R}} \\
\hat{\psi}_{n}^{\mathrm{R}}
\end{array}\right)=i \omega_{n}\left(\begin{array}{c}
\hat{\phi}_{n}^{\mathrm{R}} \\
\hat{\psi}_{n}^{\mathrm{R}}
\end{array}\right), \quad\left(\begin{array}{c|c}
0 & 1 \\
\hline L_{1} & L_{2}+L_{2}^{\partial}
\end{array}\right)\left(\begin{array}{c}
\hat{\phi}_{n}^{\mathrm{L}} \\
\hat{\psi}_{n}^{\mathrm{L}}
\end{array}\right)=i \bar{\omega}_{n}\left(\begin{array}{c}
\hat{\phi}_{n}^{\mathrm{L}} \\
\hat{\psi}_{n}^{\mathrm{L}}
\end{array}\right) .
$$


Notice that here the functions $\phi_{n}^{\mathrm{L}, \mathrm{R}}$ and $\hat{\psi}_{n}^{\mathrm{L}, \mathrm{R}}$ are, respectively, the first and second components of the left and right $\hat{w}_{n}$ and $\hat{v}_{n}$ vectors, and the "hat" indicate ("simultaneous") normalization of the 2-component vector with the energy norm $\|\cdot\|_{E}$

$$
\left\langle\left(\begin{array}{c}
\hat{\phi}_{n}^{\mathrm{L}, \mathrm{R}} \\
\hat{\psi}_{n}^{\mathrm{L}, \mathrm{R}}
\end{array}\right),\left(\begin{array}{c}
\hat{\phi}_{m}^{\mathrm{L}, \mathrm{R}} \\
\hat{\phi}_{m}^{\mathrm{L}, \mathrm{R}}
\end{array}\right)\right\rangle_{E}=\delta_{n m}
$$

Now, we can express the QNM resonant expansion (153) in terms of $\phi$ and $\psi$ as

$$
\left(\begin{array}{c}
\phi \\
\psi
\end{array}\right) \sim \sum_{n} e^{i \omega_{j} \tau} a_{n}\left(\begin{array}{c}
\hat{\phi}_{j}^{R} \\
\hat{\psi}_{j}^{R}
\end{array}\right)
$$

with the coefficient $a_{n}$ given by

$$
a_{j}=\kappa_{j}\left\langle\hat{w}_{j} \mid S\right\rangle_{E}=\kappa_{j}\left\langle\left(\begin{array}{c}
\hat{\phi}_{j}^{L} \\
\hat{\psi}_{j}^{L}
\end{array}\right),\left(\begin{array}{c}
\varphi_{0} \\
\varphi_{1}
\end{array}\right)\right\rangle_{E} .
$$

This can be made more explicit by inserting the expression for the scalar product in 155

$$
a_{j}=\frac{\kappa_{j}}{2} \int_{\Sigma}\left(w(x) \overline{\hat{\psi}_{j}^{L}} \varphi_{1}+p(x) \overline{\nabla \hat{\phi}_{j}^{L}} \cdot \nabla \varphi_{0}+\tilde{V} \overline{\hat{\phi}_{j}^{L}} \varphi_{0}\right) d \Sigma,
$$

and using now, from the first component in the spectral problem for left eigenvectors in equation (158), the relation $\hat{\psi}_{j}^{L}=i \bar{\omega} \hat{\phi}_{j}^{L}$, we have

$$
\begin{aligned}
a_{j} & =\frac{\kappa_{j}}{2}\left(\int_{\Sigma} w(x) \overline{i \bar{\omega}_{j} \hat{\phi}_{j}^{L}} \varphi_{1} d \Sigma+\int_{\Sigma}\left(p(x) \overline{\nabla \hat{\phi}_{j}^{L}} \cdot \nabla \varphi_{0}+\tilde{V} \overline{\hat{\phi}_{j}^{L}} \varphi_{0}\right) d \Sigma\right) \\
& =\frac{\kappa_{j}}{2}\left(-i \omega_{j} \int_{\Sigma} w(x) \overline{\hat{\phi}_{j}^{L}} \varphi_{1} d \Sigma+\int_{\Sigma}\left(p(x) \overline{\nabla \hat{\phi}_{j}^{L}} \cdot \nabla \varphi_{0}+\tilde{V} \overline{\hat{\phi}_{j}^{L}} \varphi_{0}\right) d \Sigma\right) \\
& =\frac{\kappa_{j}}{2}\left(-i \omega_{j}\left\langle\hat{\phi}_{j}^{L}, \varphi_{1}\right\rangle_{(2, w)}+\left\langle\hat{\phi}_{j}^{L}, \varphi_{0}\right\rangle_{H_{(V, p)}^{1}}\right) .
\end{aligned}
$$

Then, putting together the first component of equation 160 and the expression of $a_{j}$ in 163

$$
\begin{aligned}
& \phi(\tau, x) \sim \sum_{n} e^{i \omega_{j} \tau} a_{n} \hat{\phi}_{j}^{R}(x), \quad \phi(t, x) \sim \sum_{n} e^{i \omega_{j} t} a_{n} e^{i \omega_{j} h(x)} \hat{\phi}_{j}^{R}(x) \\
& \text { with } a_{j}=\frac{\kappa_{j}}{2}\left(\left\langle\hat{\phi}_{j}^{L}, \varphi_{0}\right\rangle_{H_{(V, p)}^{1}}-i \omega_{j}\left\langle\hat{\phi}_{j}^{L}, \varphi_{1}\right\rangle_{(2, w)}\right),
\end{aligned}
$$

where the factors $e^{i \omega_{j} h(x)}$ in the "Cauchy" expression $\phi(t, x)$ follow from the height function $h(x)$ in the transformation (61) from Cauchy to hyperboloidal slices $\S$ These expressions provide a QNM resonant expansion for the scattered field $\phi(\tau, x)$ whose form reduces to that of normal modes in the selfadjoint case. Specifically, in the selfadjoint case we have $\hat{\phi}_{j}^{L}=\hat{\phi}_{j}^{R}=\hat{\phi}_{j}$ and $\kappa_{j} \rightarrow 1$ and we find in a compact domain $D$ in a "Cauchy slice" (the factor $e^{i \omega_{j} h(x)}$ is absent in the already normalizable Cauchy selfadjoint case)

$$
\phi(t, x)=\sum_{j \in \mathbb{Z}^{*}} e^{i \omega_{j} t} \frac{1}{2}\left(\left\langle\hat{\phi}_{j}, \varphi_{0}\right\rangle_{H_{V}^{1}}-i \omega_{j}\left\langle\hat{\phi}_{j}, \varphi_{1}\right\rangle_{2}\right) \hat{\phi}_{j}(x),
$$

$\S$ Note that, given $\operatorname{Im}\left(\omega_{j}\right)>0$ and the structure of $h(x)$ at $\pm \infty$, the factors $e^{i \omega_{j} h(x)}$ explode at infinity. This is the signature of the lack of normalizability of QNMs in the Cauchy description. It is when using the hyperboloidal slice, and therefore a time $\tau$ that asymptotes to "retarded" and "advanced" times, that we have finite spatial functions. 
where the $\hat{\phi}_{j}$ are still normalized with respect to the energy scalar product $\langle\cdot, \cdot\rangle_{E}$. This last expression reduces indeed to the standard expression (e.g. equation (2.52) in [115])), in terms of eigenfunctions $\phi_{j}$ of $P_{V}:=-\Delta+V$ (with homogeneous Dirichlet conditions) normalized with respect to the $L^{2}$ norm (see [1]) for details)

$$
\phi(t, x)=\sum_{j \in \mathbb{Z}^{*}} e^{i \omega_{j} t} \frac{1}{2}\left(\left\langle\phi_{j}, \varphi_{0}\right\rangle_{2}-\frac{i}{\omega_{j}}\left\langle\phi_{j}, \varphi_{1}\right\rangle_{2}\right) \phi_{j}(x)
$$

The crucial difference between equations (165) and (166), on the one hand, and equation (164) is the sense of the "sum" symbol: whereas in equations $(165)$ and (166) this corresponds to a convergent series and the set of normal modes $\left\{\phi_{j}\right\}_{j \in \mathbb{N}}$ is an actual Hilbert basis, in the case of equation (164) this is just an asymptotic expansion in the sense of equations (149), 150 and 151. More generally, coming back to the non-selfadjoint case, equation (164) provides exactly the version in the Keldysh setting of the Lax-Phillips asymptotic resonant expansion. Indeed, equation (164) recovers the structure of Lax-Phillips expansion (cf. e.g. equation (2.50) in [115]; note the sign change in the convention for the spectral parameter), in particular (crucially) recovering the correct dependence in $\omega_{j}$, and not in the conjugated $\overline{\omega_{j}}$, a non-trivial check result of a subtle intertwining of the Hilbert space elements in the Keldysh's setting. Finally, in contrast with the selfadjoint case, the expression of $a_{n}$ cannot be further reduced to purely $L^{2}$ scalar products, since $\hat{\phi}_{j}^{L}$ is not the eigenfunction of the operator $P_{V}$.

\section{QNM spectral instability: an emerging general picture}

The BH instability phenomenon identified by Nollert and Price in [79, 81] has been recently revisited in [53, 24, 88, 71, 54, 26] — see also results in section 4.1.3. In particular, from the work in [53, 54] a consistent picture of this phenomenon emerges, in which the fundamental QNMs are stable under arbitrary perturbations (respecting the asymptotic structure) whereas overtones are unstable under high-frequency perturbations. In this section we discuss first the main ingredients building our current understanding of the problem and then detail a description of the emerging picture. We close the section by considering some possible implications of such general picture for BH spectroscopy in gravitational wave astronomy.

\subsection{Elements in the QNM spectral instability problem}

5.1.1. QNM-free regions: logarithmic boundaries and pseudospectra. As discussed in sections 2.1 and 4.1. the calculation of QNMs in the hyperbolic approach is cast in terms of the eigenvalue problem of a non-selfadjoint operator $L$. In this setting, QNM instability is reduced to the study of the spectral instability of such an operator. Crucially, this can be studied at the level of the original unperturbed operator $L$, without implementing the actual perturbations $\delta L$. Specifically, as presented in section 2.1.1 and the applied in the example in section 3.1.2, the notion of pseudospectrum (see [105, 96] and references in [53]) provides an approach to assess such instability, in terms of the analytical properties of the resolvent of $L$.

When the operator $L$ is perturbed, its eigenvalues can migrate inside specific regions in the complex plane around the original spectrum $\sigma(L)$, depending on the size (norm) of the enforced perturbations. If such possible regions are concentrated around $\sigma(L)$, the operator is spectrally stable but if, on the contrary, such regions are well extended in $\mathbb{C}$, then large instabilities can show up. More specifically — applying the first characterization in equation (7) to QNM frequencies $\omega$ - the QNM $\epsilon$-pseudospectrum $\sigma^{\epsilon}(L)$ region is the set of complex QNM frequencies that are actual resonances of some perturbed operator — defining 
the perturbed scattering problem — whose difference $\delta L$ with the original one is $O(\epsilon)$, that is

$$
\sigma^{\epsilon}(L)=\left\{\omega \in \mathbb{C}, \exists \delta L \in M_{n}(\mathbb{C}),\|\delta L\|<\epsilon: \lambda \in \sigma(L+\delta L)\right\} .
$$

The $\epsilon$-pseudospectrum $\sigma^{\epsilon}(L)$ is then the maximal region in the complex plane that QNMs can reach under perturbations of norm $\epsilon$. Then, for a given $\epsilon$, the regions beyond the $\epsilon$ pseudospectrum boundaries are QNM-free, or resonance-free, regions in the complex plane.

In the setting of Schwarzschild's potential, the results in [54] (see also [26] in ReissnerNordström) permit to identify the resonance-free region from the numerically calculated pseudospectra, concluding that such QNM-free regions are asymptotically bounded below by logarithmic curves of the form (here $C_{1}, C_{2}$ and $C_{3}$ are constants)

$$
\operatorname{Im}(\omega) \sim C_{1}+C_{2} \ln \left(\operatorname{Re}(\omega)+C_{3}\right),|\operatorname{Re}(\omega)| \gg 1 .
$$

This is consistent with theoretical analytical results. Indeed, logarithmic lower bounds $\operatorname{Im}(\omega) \sim M \ln \operatorname{Re}(\omega)$ of resonant-free regions appear in the setting of scattering by obstacles (where the field does not penetrate the scatterer) and smooth compact support potentials, as shown by Regge [90], Lax \& Phillips [68, 67] and Vainberg [107] (see [115, 29] for a detailed discussion and references). This generalizes to (non-trapping) smooth non-compact support potentials [95, 77, 99]. The interesting result is that in the Schwarzschild (also Reissner-Nordström and Pöschl-Teller) case, such asymptotics extend far from the asymptotic region into the "inner" region around non-perturbed QNMs, if we allow for a translation in the imaginary and real parts of $\omega$-respectively $C_{1}$ and $C_{3}$ - that disappear at large asymptotics. This asymptotically logarithmic shape of the QNM-free region, determined from the pseudospectrum, is the first element in our general QNM spectral instability picture.

5.1.2. Regular high-frequency QNM perturbations: Nollert-Price-like branches and "inner" QNMs. The second element building a general picture of QNM instability concerns the migration of QNMs to perturbed QNM branches. Consistently with the pseudospectrum analysis discussed above, perturbed QNMs corresponding to $\|\delta L\|<\epsilon$ must lay above the $\epsilon$-pseudospectrum contour lines in $\mathbb{C}$. Given the large size of pseudospectra logarithmic regions in our problem, this is not a tight constraint and, indeed, low frequency perturbed QNMs stay far from pseudospectra boundaries. Instability is triggered only by high-frequency perturbations, that leave stable the fundamental QNM but push overtones to open branches that we generically refer as Nollert-Price branches, to acknowledge the first identification of this phenomenon in [79, 81] (although different types of branches can and do appear [54]).

Interestingly, as discussed in [54], several new "Nollert-Price" branches can appear under perturbations, depending on the size and frequency of the latter. The passage from one such branch to another in the complex plane is marked by the presence of new QNMs in the "interior" of the new branches, typically less damped and with smaller oscillation frequency. Such "inner QNMs" do not place themselves along structured branches, but rather seem to populate the region in the interior of the open branches. These inner QNMs are indeed eigenvalues of the operator $L+\delta L$ (they pass a convergence test) and are very sensitive to the applied perturbation. Their role in the QNM instability picture remains to be elucidated.

In generic terms, the relation between the pseudospectrum and the perturbation of QNMs frequencies can be understood in terms of the Bauer-Fike theorem. Given an operator $A$, the 'tubular neighborhood' $\Delta_{\epsilon}(A)$ of radius $\epsilon$ around the spectrum $\sigma(A)$, defined as

$$
\Delta_{\epsilon}(A)=\{\lambda \in \mathbb{C}: \operatorname{dist}(\lambda, \sigma(A))<\epsilon\},
$$


is, by definition of the pseudospectrum, always contained in the $\epsilon$-pseudospectrum $\sigma^{\epsilon}(A)[105$

$$
\Delta_{\epsilon}(A) \subseteq \sigma^{\epsilon}(A) .
$$

Therefore, the question is how far the tubular neighborhood of radius $\epsilon$ stays away from the pseudospectrum boundary. Selfadjoint (more generally, normal operators) indeed satisfy [105

$$
\sigma_{2}^{\epsilon}(A)=\Delta_{\epsilon}(A),
$$

where $\sigma_{2}^{\epsilon}(A)$ indicates the use of a $\|\cdot\|_{2}$ norm. As a consequence, pseudospectra of normal operators show characteristic concentric 'circles' tightly packed around the eigenvalues. When moving away from normality, the instability of a given eigenvalue $\lambda_{n}$ is controlled by the so-called condition number $\kappa_{n}$ introduced in equation (128), through the inequality (6). In the normal case, $\kappa_{n}=1$. However, in the non-normal case $\kappa\left(\lambda_{i}\right)>1$, and can grow unbounded if left- and right- eigenvectors in $(128)$ tend towards orthogonality. The essential content of Bauer-Fike theorem —cf. [105]—is the identification of such $\kappa_{n}$ numbers as the key element in the relation between pseudospectra and eigenvalue perturbations, by determining the effective radii in which perturbed QNMs can move around the spectrum

$$
\sigma^{\epsilon}(A) \subseteq \Delta_{\epsilon \kappa}(A):=\bigcup_{\lambda_{i} \in \sigma(A)} \Delta_{\epsilon \kappa\left(\lambda_{i}\right)+O\left(\epsilon^{2}\right)}\left(\left\{\lambda_{i}\right\}\right) .
$$

In sum, QNM branches whose eigenvalues have large condition numbers $\kappa_{n}$ are subject to potential instabilities. In contrast, branches with low $\kappa_{n}$ remain stable under perturbations.

In our problem, non-perturbed $\mathrm{BH}$ QNM overtones have large $\kappa_{n}$ [53], consistently with the large logarithmic pseudospectra regions. They are therefore potentially unstable under physical perturbations. However, this does not mean that any perturbation triggers the instability. In fact, low frequency perturbations do not modify QNMs in an unstable way, but rather spectrum modifications are of the same scale as that perturbation. On the contrary, high energy perturbations lead to perturbation patterns (mainly 1 ) structured in the new branches we have referred to as Nollert-Price branches, sharing the following qualitative points:

i) Perturbed QNMs became less damped, lasting longer in time, therefore $\operatorname{Im}\left(\omega_{n}\right)$ reduces.

ii) Oscillations probe smaller scales, specifically $\operatorname{Re}\left(\omega_{n}\right)$ is not bounded above.

iii) As a result, perturbed QNM branches show a characteristic opening in the complex plane, growing from a first perturbed QNM overtone in the non-perturbed QNM spectrum.

iv) The first perturbed overtone gets closer to the real axis as the frequency and/or the size of the perturbation increases. The fundamental QNM always stay stable under this type of low-amplitude and high-frequency perturbation

v) The resulting perturbed QNM branches are stable under further perturbations, accordingly perturbed QNMs have lower condition numbers $\kappa_{n}$.

† Notice also the mentioned presence of "inner QNMs", in the interior region of these branches and not along them. This different pattern may respond to a distinct underlying instability mechanism.

$\ddagger$ The high-frequency perturbations respect a "non-trapping condition" for the perturbed potential, namely there are not closed trajectories in the phase space - apart from the "point trajectory" corresponding to the unstable equilibrium at the (single) maximum of the potential. If we allow for closed trajectories in the unperturbed potential, such as the "well in an island" potential [15] with two maxima, then perturbations of the fundamental QNM can occur even for very tiny double potentials ("flea on the elephant" effect [57, 40, 93]). We thank V. Cardoso for signalling this point. 
Such qualitative behaviour of QNMs is compatible with the generic one found in the scattering by matter compact objects, namely $w$-modes —or curvature QNMs [61] - or in QNMs of compact obstacles. This 'universality', however, is not very constraining concerning the shapes of the perturbed branches, specifically in the asymptotic large frequency regime. To illustrate this, we mention the case of convex obstacles, where the asymptotic form of QNM branches (under a 'pinched curvature assumption') can be explicitly established [98, 114]

$$
\operatorname{Im}\left(\omega_{n}\right) \sim K\left|\operatorname{Re}\left(\omega_{n}\right)\right|^{\frac{1}{3}}+C, n \gg 1 .
$$

Such QNM power-law behavior follows the boundary of resonant-free regions for scatterers whose regularity is of Gevrey type. Specifically $s$-Gevrey classes interpolate between analyticity, corresponding to $s=1$, and $C^{\infty}$ regularity, for $s=\infty$. The asymptotic behavior of the resonant free regions for scatterers of $s$-Gevrey class is given by $\operatorname{Im}(\omega)>K \omega^{1 / s}[39$, consistent with the actual Gevrey $s=3$ regularity in the example in (173). In our BH setting, the analysis in [33, 34, 35] suggests a special role for Gevrey $s=2$ classes $\S$ and, therefore, branches of type $\operatorname{Im}\left(\omega_{n}\right) \sim K\left|\operatorname{Re}\left(\omega_{n}\right)\right|^{\frac{1}{2}}$. However, the numerical methodology adopted in [53,54] makes very difficult to determine the precise asymptotics and, actually, branches with different detailed behaviors are observed [54] — together with the "inner QNMs" marking the passage between such different branches. In spite of the resulting complexity and the numerical difficulties for the study of large- $n$ asymptotics, a remarkable regularity shows up: QNMs on Nollert-Price branches tend to place themselves uniformly along the branch, this ultimately leading to a Weyl law for the asymptotic counting of QNMs || Inner QNMs do not change such Weyl law, though they induce a transition in the relevant length scale [54].

Beyond the complexity of QNM Nollert-Price branches, the (Gevrey) example in equation (173) illustrates a fundamental point: in setting we are studying, (a part of) perturbed QNMs tend to migrate towards the pseudospectra boundaries. This is systematically observed in the numerical studies in [53, 54]. In spite of the difficulties to study numerically QNM asymptotics, on can soundly conclude: as long as perturbations are regular (at least, $C^{\infty}$ ), they stay distinctly above the corresponding $\epsilon$-pseudospectrum asymptotically logarithmic lines. That is, perturbed QNM tend to track pseudospectrum lines, getting closer to them as frequency increases but always staying above as long as perturbations are at least $C^{\infty}$. This is the second element in our general picture of high-frequency QNM spectral instability.

5.1.3. Non-regular $C^{p}$ QNM perturbations: logarithm Regge branches. When $C^{\infty}$ regularity is lost, perturbed QNMs reach the logarithmic boundaries of the QNM-free regions. This is the third key ingredient in our picture of QNM instability picture. Perturbations with

$\S$ Note that in our QNM stability discussion, a special role is played by the energy scalar product and the associated energy norm (namely a $H^{1}$-like norm). This is because we privilege "energy" as as measure of the size of the perturbations. If we are rather interested in the QNN definition — a problem of different nature to QNM stability$H^{1}$-like norms are not sufficient to characterize the spectrum. Specifically, a stronger "control" of the regularity of eigenfunctions associated with QNMs is needed, as the one provided by Gevrey classes (controlling higher derivatives with appropriate weights). In pseudospectrum terms, this would amount to much tighter $\epsilon$-pseudospectrum lines, defining the spectrum in the $\epsilon \rightarrow 0$ limit. In other words, one would use first Gevrey classes to define QNMs in both the non-perturbed and the perturbed potentials and, in a second step, one would use the energy norm to assess if the associated operator perturbations - possibly inducing QNM instabilities — are actually big or small (in energy terms). The relation between the two problems above is fundamental and requires further specific investigation.

\| This suggests the possibility of extracting fine-structure information from fluctuations (with respect to the 'regular unfolding' of the spectrum, using Weyl law's), by adopting a 'spectrum statistics' approach to the QNM spectrum properties (cf. e.g. [86] and references therein). It is also interesting to note that, together with the different 'open branch' Nollert-Price regimes, other 'non-branch' behavior associated with 'inner QNMs' show up. Such inner QNMs seem to follow well-defined probabilistic distributions in $\mathbb{C}$ (the latter entering in the spectral analysis of perturbation of non-selfadjoint operators [96]), suggesting the need to import stochastic tools to the study of QNMs. 
$C^{p}$ regularity, i.e. with a discontinuous $p$-th derivative (for any $p<\infty$ ) make QNM migrate to branches that saturate the pseudospectrum boundaries, following logarithmic lines 168. Specifically, $C^{p<\infty}$-perturbed QNMs follow Regge QNM branches [90, 15, 115, 29]

$$
\begin{aligned}
& \operatorname{Re}\left(\omega_{n}\right) \sim \pm\left(\frac{\pi}{L} n+\frac{\pi \gamma_{p}}{2 L}\right) \\
& \operatorname{Im}\left(\omega_{n}\right) \sim \frac{1}{L}\left[\gamma \ln \left(\left(\frac{\pi}{L} n+\frac{\pi \gamma_{p}}{2 L}\right)+\frac{\pi \gamma_{\Delta}}{2 L}\right)-\ln S\right],
\end{aligned}
$$

for $n \gg 1$. This asymptotic result, first found by Regge [90] has been independently recovered later in different settings. Note that such branches place themselves on the top of asymptotic pseudospectrum lines $\left[168\right.$, with $C_{1}=-\ln (S) / L, C_{2}=\gamma / L$ and $C_{3}=\pi \gamma_{\Delta} /(2 L)$.

In the setting of compact-support potentials (actually the context in Regge's work [90]), this result has been discussed in full detail by Zworski [113]. Specifically, considering a potential with compact support in the interval $[a, b]$ and with behavior

$$
\begin{array}{lll}
V(x) \sim V_{1}(x-a)^{m} & , & \text { as } x \rightarrow a^{+} \\
V(x) \sim V_{2}(x-b)^{k} & , & \text { as } x \rightarrow b^{-}
\end{array}
$$

with $m, k$ non-negative integers, the QNMs follow Regge branches 174 with

$$
\begin{aligned}
& L=(b-a), \gamma=\frac{m+k+4}{2} \\
& \gamma_{p}=\gamma+\left(1+\operatorname{sign}\left(V_{1} V_{2}\right)\right), \gamma_{\Delta}=-\gamma_{p} \\
& S=\left(\frac{\left|V_{1} V_{2}\right| k ! m !(k+m+2)}{2^{m+k+1}}\right)^{\frac{1}{2}} .
\end{aligned}
$$

This rigorous result fully accounts for the branches found by Nollert in [79], since the stepwise potential there employed to approach Schwarzschild is, in particular, a compact-support $C^{0}$ potential. Interestingly, the same theorem states that QNMs of smooth $\left(C^{\infty}\right)$ class must lay strictly above the logarithmic lines, consistently with the pseudospectrum analysis in section 5.1.1. The result does not directly apply to the 'spiked truncated dipole' potential in [81], since this is a non-compact support potential with a finite jump at $x=x_{0}$ and a Diracdelta perturbation at $x=x_{\delta}$, namely $\delta V=V_{o} \delta\left(x_{\delta}\right)$. In spite of the non-compactness, the asymptotic analysis by Nollert and Price leads exactly to Regge branches (174), now with

$$
L=\left(x_{\delta}-x_{0}\right), \gamma=\gamma_{p}=\frac{3}{2}, \gamma_{\Delta}=0, S=\left(\frac{V_{\delta}}{4 x_{0}^{2}}\right)^{\frac{1}{3}} .
$$

More generally, a higher-order WKB approach [8, 9] can be envisaged to capture the QNM asymptotics of general (non-compact) $C^{p<\infty}$ potential barriers. This approach boils down to the determination by Berry [8] of the "reflection coefficient" $R$ at a point $x=x_{0}$, where the potential $V$ is discontinuous in the $p$ th-derivative (cf. equation (27) in [8]), namely

$$
R \sim\left(\lim _{x \rightarrow x_{0}^{+}} \frac{d^{p} V}{d x^{p}}-\lim _{x \rightarrow x_{0}^{-}} \frac{d^{p} V}{d x^{p}}\right):=\Delta_{p} V .
$$

This WKB approach has been applied in Zhang et al. [112] to recover Regge branch asymptotics of polytropic neutron stars (with $L$ the "tortoise" radius of the star, $\gamma=p+2$ fixed by the polytropic index, $\gamma_{p}=\theta_{b}$ depending on the wave parity, $\gamma_{\Delta}=0$ and $S$ proportional to $\Delta_{p} V$ ) and in Qian et al. [88, 71] for BH-like potentials with a "jump" or "cut" ( $L$ is there given in terms of the position of the jump $x_{\text {cut }}$ and $S$ is again controlled by $\Delta_{p} V$ ). 
In sum, QNMs of $C^{p}$ potentials follow logarithm Regge branches that saturate the pseudospectrum boundary lines. In particular, QNMs in [79] are indeed Regge branches \. The explicit models behind Regge branches lead to an interpretation of the coefficients [54]:

i) “Length scale" $L$ : straightforwardly obtained as $L=\pi\left(\operatorname{Re}\left(\omega_{n+1}\right)-\operatorname{Re}\left(\omega_{n}\right)\right)^{-1}$, it is related to a 'bouncing' scale [90], as illustrated by $L=(b-a)$ in [113] or $L=x_{\delta}-x_{0}$ in [81] (in [71] $T=2 L$ has been proposed as a bouncing time in an "echoes" mechanism).

ii) "Small structure" coefficient $\gamma$ : this coefficient is explicit identified in [113] with the regularity of the perturbations. This raises the question: can one read the (effective) regularity of spacetime from QNM observational data?

More soberly, we refer to this parameter as a "small structure" coefficient, something further supported in a matter setting, where $\gamma$ is —as mentioned above- directly related in [112] to the polytropic index in the equation of state of polytropic (neutron) stars. This parameter should strongly depend on the frequency of the underlying perturbation.

iii) "Strength coefficient" $S$ (or "imaginary shift"): this parameter is directly related to the "size" of the perturbation, namely $V_{1}$ and $V_{2}$ in equation [175] in [113], $V_{\delta}$ in [81] or $\Delta_{p}$ in equation (177) used in [112, 88]. However, it also depends on the regularity/frequency of the perturbation and the size and frequency effects can be difficult to disentangle. In this sense, it should be determined after $\gamma$. The term $\ln (S) / L$ provides a translation in the imaginary part in the $\operatorname{Im}\left(\omega_{n}\right)=f\left(\operatorname{Re}\left(\omega_{n}\right)\right)$ functional dependence.

iv) "Parity term" $\gamma_{p}$ : it shifts the location of Regge QNMs along the logarithmic branches according to sign/parity properties of the potential. In [113] it depends on the relative sign of $V_{1}$ and $V_{2}$, whereas in [112] it depends on the axial/polar character of the perturbation. It seems to play a role in the "polarity-alternant" pattern in $w$-like modes.

v) "Real shift" $\gamma_{\Delta}$ : it induces a translation in the real part of the relation $\operatorname{Im}\left(\omega_{n}\right)=$ $f\left(\operatorname{Re}\left(\omega_{n}\right)\right)$ determining, with $\ln (S) / L$, the first overtone affected by a perturbation.

The $C^{p}$ potentials here considered provide an instance of (infinite) ultraviolet limit "highfrequency" spacetime perturbation. They are not only logarithmic, but they also provide a very specific form of the QNM distribution along that logarithmic branches. A natural question is posed: how generic is such Regge QNM distribution in the ultraviolet perturbation limit?

\subsection{Emerging picture: universality in QNMs of compact objects}

The previous points build a consistent picture for the BH QNM instability pattern:

i) The fundamental BH QNM (of each $\ell$-fixed branch) is stable under (generic "nontrapping") perturbations respecting the asymptotic structure at null infinity.

ii) QNM-free regions are bounded-above by logarithmic curves, namely the pseudospectra contour line boundaries.

iii) Generic regular $\left(C^{\infty}\right)$ high-frequency perturbations make QNM overtones migrate to either a) Nollert-Price-like branches whose opening is associated with a "bouncing/reverberance" mechanism, or b) "inner QNMs" marking the transition between different perturbed branches. As long as the perturbation frequency stays finite, perturbed QNMs lay (strictly) above pseudospectrum logarithmic curves.

iv) Low-regularity $C^{p}$ perturbations, in particular involving an (ultraviolet) infinite highenergy limit, are 'optimal' in reaching the logarithmic QNM-free region boundaries.

व We prefer in any case use the term Nollert-Price branches to all perturbed branches (even not logarithmic as those in section 5.1.2, to stress the first authors that identified the QNM instability branch opening phenomenon. 
v) Perturbed QNMs migrated to Nollert-Price-like branches are stable under further perturbations, whereas "inner QNMs" are highly unstable, distributing in "transition regions" in the interior of Nollert-Price-like branches.

vi) The resulting perturbed $\mathrm{BH}$ Nollert-Price QNM branches share some of the qualitative features of QNM branch patterns of matter compact objects: high-frequency perturbations induce a transition from the non-perturbed BH (structurally unstable) QNM branches to new pertubed (structurally stable) QNM branches qualitatively similar for all (vacuum or matter) compact objects, pointing towards a possible universality [53].

Some remarks concerning the previous heuristic picture:

a) The (in)stability of QNMs is claimed under generic perturbations. This does not preclude that certain fine-tuned perturbations can induce specific non-generic behaviors. This may be relevant in specific models.

b) The asymptotics of generic ultraviolet perturbations, when the perturbation frequency tends to infinity in an arbitrary way, is a point one would like to elucidate. As stated in point iv) above, the enforcement of $C^{p}$ perturbations realizes an instance of such ultraviolet situation, but not as a limiting process. This issue will be revisited in section 6.2. after in introducing in section 6.1 an additional piece of information, leading to the conjecture that arbitrary infinite high-frequency limits lead to a $C^{p}$ state, so Regge QNMs are the precise and generic QNM pattern of generic ultraviolet perturbations.

c) The nature of the "inner QNMs" and the associated Nollert-Price-like branch transitions observed in [54] remains quite elusive. An understanding of this problem could shed light on the different scales involved in the QNM instability problem.

\subsection{BH spectroscopy and $\epsilon$-dual QNM resonant expansions.}

Let us consider a non-perturbed BH with non-perturbed QNMs $\omega_{n}$. On the one hand, section 5 provides a description of the QNM spectral instability phenomenon, according to which high-frequency perturbations with $\|\delta L\|=\epsilon$ perturb the QNMs to very different new values $\omega_{n}^{\epsilon}$. Beyond the "frequency-domain" spectral computations in [53, 54], the "time-domain" results in [54] crucially confirm the presence of such perturbed QNMs $\omega_{n}^{\epsilon}$ in the time signal.

On the other hand, in section 4.2 we have constructed resonant expansions of the timedependent field scattered by a given potential in terms of the QNMs of that potential. Given i) a non-perturbed potential $V$ with $\hat{v}_{n}(x)$ and $\omega_{n}$ QNM functions and frequencies, and ii) a perturbed potential $V^{\epsilon}=V+\epsilon \delta V$ (with $\|\delta V\|_{E}=1$ ) with $\hat{v}_{n}^{\epsilon}(x)$ and $\omega_{n}^{\epsilon}$ QNMs, we can write the respective scattered fields $u(\tau, x)$ and $u^{\epsilon}(\tau, x)$ in QNM resonant expansions as

$$
u(\tau, x) \sim \sum_{n} e^{i \omega_{n} \tau} a_{n} \hat{v}_{n}(x) \quad, \quad u^{\epsilon}(\tau, x) \sim \sum_{n} e^{i \omega_{n}^{\epsilon} \tau} a_{n}^{\epsilon} \hat{v}_{n}^{\epsilon}(x)
$$

in accordance with the resonant expansion structure in general scattering theory [67, 107, 29].

5.3.1. GW signal time domain stability versus QNM spectral instability. In this setting, let us note the stark contrast in the stability — based on the same energy norm $\|\cdot\|_{E}$ - of the "frequency domain" and "time domain" problems associated with $L$ :

i) Spectral QNM problem of $L$, equation 140): instability in the energy norm. As reviewed in section 5 , this problem is strongly unstable under high-frequency perturbations. 
ii) Time evolution problem of $L$, equation (133): stability in the energy norm. As discussed in [79, 81] and later revisited in [24, 88], time evolution signals $u(\tau, x)$ and $u^{\epsilon}(\tau, x)$ "look very similar" so the dynamical problem seems stable in spite of the strong QNM spectral instability. This can be understood on general grounds from (finite) energy considerations and from the absence of unstable QNMs. In particular, it can be better assessed in terms of the stability of this dynamical problem in the energy norm $\square$ From the Cauchy problem 133 for the non-perturbed potential, one can write

$$
\left\|u-u^{\epsilon}\right\|_{E} \lesssim \bar{C}\|u\|_{E} \epsilon \lesssim C \epsilon,
$$

that provides a crude (energy) estimate of the difference between the unperturbed and perturbed time signal. Certainly this is not the pointlike estimate for the time series at null infinity (namely, the idealized position of the detector) that one would need to compare the observed signals, but it gives an idea that for sufficiently regular time signals their difference is proportional to the perturbation size, then providing a notion of stability.

There is no actual contradiction in such radically different stability behaviour (in the energy norm ${ }^{*}$ of these two related, but different, problems associated with $L$. But this stark contrast suggests the following question: writing formally, from the crude energy estimation [179] and for sufficiently regular time signals, the approximation

$$
u^{\epsilon}(\tau, x) \sim u(\tau, x)+O(\epsilon)
$$

then, writing the respective QNM resonant exoansions (178), we have

$$
\sum_{n} e^{i \omega_{n}^{\epsilon} \tau} a_{n}^{\epsilon} \hat{v}_{n}^{\epsilon}(x) \sim \sum_{n} e^{i \omega_{n} \tau} a_{n} \hat{v}_{n}(x)+O(\epsilon) .
$$

In other words, in a physical situation with perturbations, the perturbed field $u^{\epsilon}(\tau, x)$ admits an "exact" resonant expansion in terms of the perturbed QNMs $\omega_{n}^{\epsilon}$, but also an expansion in terms of non-perturbed QNMs $\omega_{n}$ that is indistinguishable (and therefore valid) as long as energies involved are above or of the order of the energy $\epsilon$ of the triggering perturbation.

Then, which expansion should one use? The message we would like to convey is that both can and must be employed: whereas non-perturbed QNMs encode information about the large scale properties of the $\mathrm{BH}$, the perturbed QNM expansion encodes information on the perturbation physics. We refer to QNM-expansions in (181) as ' $\epsilon$-dual QNM expansions'.

\footnotetext{
+ Note that we refer here to stability upon perturbations $\delta L$ of the operator $L$ defining the dynamics. This notion of stability is different from that of the usual PDE perspective, focused on analysing the stability of solutions $u(t, x)$ of a given fixed equation upon perturbations $\delta u_{0}(x)$ of the initial data with respect to reference initial data $u_{0}(x)$.

* If one wants to better control the regularity of the signal to give a better estimate than the one in equation [179, one would need to use rather norms controlling higher derivatives, namely Sobolev norms in $W^{k, p}$. In the setting of this work where the scalar product plays a key role is natural to choose Hilbert spaces $H^{k}=W^{k, 2}$. Interestingly, given that the QNM instability is a high-frequency phenomenon, we can expect significant differences in the signal at high-wave numbers. Then, in terms of a high- $k$ Sobolev norm the difference $\left\|u-u^{\epsilon}\right\|_{H^{k}}$ could be much larger than $\epsilon$, consistently with an instability in the time-domain signal. So both the spectral and time domain problem would be unstable under high-frequency perturbations, when measuring the operator pertubation in the energy norm.

One could also think the problem alternatively, by changing the manner of measuring the size of the operaror perturbation $\delta L$ : if we choose the operator norm induced from $\|\cdot\|_{H}$, a high-frequency perturbation $\delta L$ that we would interpret as small in the energy norm ( $H^{2}$-like norm) would be actually very large in the Sobolev norm (i.e. $\|\delta L\|_{H^{k}}>>\|\delta L\|_{E}$ for high-frequency perturbations and sufficiently high $k$ ), so both the large perturbations in the eigenvalues and the large $\left\|u-u^{\epsilon}\right\|_{H^{k}}$ would be consistent with spectral stability. But such reconciliation of frequency- and time-domain stability in the $H^{k}$ norm is not very interesting in our setting, since it is only a rephrasing of the known stability under low frequency perturbations. These issues will be addressed in more detail elsewhere.
} 
5.3.2. Data analysis inverse problem: perturbation-enhanced degeneracy. If such a notion of $\epsilon$-dual QNM expansion is pertinent in the BH spectroscopy problem, then unfortunately it comes with a very non-trivial challenge data analysis: if very similar GW waveforms can actually correspond to very different sets of QNM frequencies, triggered by very tiny perturbations characterised by a large parameter space, then the data analysis inverse problem seems extremely degenerate H. Developing specifically suited strategies would be required.

Given an observed GW signal, the QNM expansion based on non-perturbed QNMs has a special feature: if it can actually be constructed, then it should minimize the number of excited modes as compared with other $\epsilon$-dual QNM expansions. The reason stems from the instability of non-perturbed QNM overtones $\omega_{n}$ - with large condition numbers are large $\kappa_{n} \gg 1$ - and the stability of already perturbed QNMs $\omega_{n}^{\epsilon}-$ with $\kappa_{n}^{\epsilon} \sim 1$. From equation 153) we can write for the expansion coefficients in equation 178

$$
a_{n}=\kappa_{n}\left\langle\hat{w}_{n} \mid u_{0}\right\rangle_{E} \quad, \quad a_{n}^{\epsilon}=\kappa_{n}^{\epsilon}\left\langle\hat{w}_{n}^{\epsilon} \mid u_{0}\right\rangle_{E} .
$$

Then, given the growth of $\kappa_{n}$ with $n$ [53], the initial data $u_{0}$ should project only on the first non-perturbed modes $\hat{w}_{n}$, to preclude the series from exploding. In contrast, since $\kappa^{\epsilon}$ are of order unity, more $\hat{w}_{n}^{\epsilon}$ can be involved. Typically, a QNM expansion in perturbed QNMs will need more terms than one in non-perturbed ones. This is consistent with results in [71, 54].

If one aims at constructing the QNM expansion of the observed GW waveform built on the perturbed QNM frequencies corresponding to the underlying perturbation, one lacks (in contrast with non-perturbed QNMs) an analytical parametrization of the exact possible solutions to be used as an "a priori" input in the data analysis extraction. In this context, by enforcing as Ansätze educated guesses for perturbed QNM branch patterns (e.g. the Regge QNM branches (174], as suggested in [54]) could render feasible the data analysis process.

Finally, if such an " $\epsilon$-dual resonant expansion" strategy can actually be implemented, it would be informative (up to precision $\epsilon$ in energy) about two complementary physics regimes: i) non-perturbed $\mathrm{BH}$ QNM expansions would retrieve the physical parameters of the underlying 'averaged BH' in a standard Kerr uniqueness paradigm, whereas ii) Nollert-Pricelike BH QNM expansions would probe the small scales of the environmental perturbations or, if Planck scales are involved, the effective regularity/granularity/stochasticity of spacetime.

\section{Geometry and QNM instability: heuristics on further structural questions}

In this section we initiate the exploration, at a heuristic level, of some of the stuctural geometric aspects underlying the BH QNM instability. In this sense, it must be understood as an invitation to a more detailed research, rather than an account of actual results.

\subsection{Physical interpretation of the energy norm: role of spacetime density perturbations}

First, we discuss the relation between the characteristics of spacetime metric perturbations and the norms of operator perturbations $\delta L$ in the eiganvalue problem (4) and (5). and the associated norms in the QNM problem of equation Since this is a heuristic argument we will use the symbol $\sim$ to denote equality where subleading terms are ignored.

The potential $V$ - and hence its rescaled version $\tilde{V}$ - appearing in equation (59) can be put in correspondence with the Ricci scalar of a conformally rescaled metric $\tilde{g}_{a b}=\Xi^{2} g_{a b}$ where $g_{a b}$ is the physical metric solving the Einstein field equations and $\Xi$ is a conformal

$\sharp$ Actually, there would be not only one pair of $\epsilon$-dual QNM expansions, but a whole (continuous) family of them parametrized by all possible perturbations of size $\epsilon$. This looks like a formidable data analysis problem. 
factor vanishing at $\mathscr{I}^{+}$(see e.g. [85]), so that formally we can write

$$
\tilde{V} \sim C+\tilde{R}
$$

where $C$ is a term associated with the angular part of the propagating field and the particular coordinates used -in the case discussed in section 3.2, this corresponds to the constant term $\ell(\ell+1)$ - which will not enter at a first order in our discussion, since we will be interested in variations of the potential related to the subjacent geometric background, so $\delta C \sim 0$ and

$$
\delta \tilde{V} \sim \delta \tilde{R}
$$

On the other hand, from a variational point of view, one can relate variations in the metric with variations in the Ricci scalar via

$$
\delta \tilde{R}=\delta\left(\tilde{g}^{a b} \tilde{R}_{a b}\right)=\tilde{R}_{a b} \delta \tilde{g}^{a b}+\tilde{g}^{a b} \delta \tilde{R}_{a b},
$$

where the conformally rescaled Ricci tensor $\tilde{R}_{a b}$ and the physical one $R_{a b}$ are related through

$$
\tilde{R}_{a b}=R_{a b}-2 \Xi^{-1} \tilde{\nabla}_{a} \tilde{\nabla}_{b} \Xi-\tilde{g}_{a b} \tilde{g}^{c d}\left(\Xi^{-1} \tilde{\nabla}_{c} \tilde{\nabla}_{d} \Xi-3 \Xi^{-2} \tilde{\nabla}_{c} \Xi \tilde{\nabla}_{d} \Xi\right),
$$

(cf. for instance [108]). Observe that $\tilde{R}_{a b}$ is formally singular at $\mathscr{I}$. Additionally, notice that

$$
\delta \tilde{g}^{a b}=\Xi^{-2} \delta g^{a b},
$$

where we have assumed that $\delta \Xi=0$, consistent with the "scri fixing" philosophy of the hyperboloidal approach. Thus overall, the first term in equation (185) is formally singular at $\mathscr{I}^{+}$unless $\delta g^{a b} \sim O\left(\Xi^{3}\right)$ close to $\mathscr{I}$ or compactly supported perturbations $\delta g^{a b}$ are considered. For the rest of this section, this latter assumption will be made so the main contribution in equation (185) comes from the second term $\dagger$ and we can write

$$
\delta \tilde{R} \sim \tilde{g}^{a b} \delta \tilde{R}_{a b}
$$

where the right hand side can be cast in the form

$$
\tilde{g}^{a b} \delta \tilde{R}_{a b}=\tilde{\nabla}^{a} v_{a}
$$

where (see e.g. [109])

$$
v_{a}=\tilde{\nabla}^{b}\left(\delta \tilde{g}_{a b}\right)-\tilde{g}^{c d} \tilde{\nabla}_{a} \delta \tilde{g}_{c d} .
$$

Hence, we can formally relate the variations in the potential to variations in the metric

$$
\delta \tilde{V} \sim \delta \tilde{R} \sim\left|\partial^{2} \delta \tilde{g}_{a b}\right| \sim \Xi^{-2}\left|\partial^{2} \delta g_{a b}\right|
$$

so that we can write

$$
\delta g \delta \tilde{V} \sim \Xi^{-2}\left|\partial \delta g_{a b}\right|^{2}
$$

where $k$ is the wave number. Therefore, given different metric perturbations, those with higher frequencies will have a stronger impact in $\delta \tilde{V}$ and, consequently, in the QNM spectrum. This is consistent with the findings in [53, 54] that identify the QNM instability phenomenon as a

$\dagger$ Another approach to justify $\delta \tilde{R} \sim \tilde{g}^{a b} \delta \tilde{R}_{a b}$ is a reminiscent of a virial-like reasoning. Indeed, the first term in equation 185 can be understood as a kinetic energy term, whereas the second one plays the role of a potential energy term. Under the assumption that the considered spacetime perturbations are virialised (in some appropriate sense to be better seized), the two terms are commensurate $\tilde{R}_{a b} \delta \tilde{g}^{a b} \sim \tilde{g}^{a b} \delta \tilde{R}_{a b}$ and 188 holds. This would be consistent (in an averaged ergodic sense) with the requirement of stationarity in the characterization and treatment of QNMs. 
high-frequency perturbation effect. It also matches the observation that in linearized gravity, if one considers the Isaacson stress-energy tensor - see [2], the energy density of the perturbed gravitational field is associated to terms quadratic in derivatives of the metric perturbation

$$
\rho_{E}(\delta g ; x) \sim|\partial \delta g|^{2}
$$

Now, let us relate this to the energy norm of the perturbations of the operator $L$. Let us consider a perturbation $\delta L$ associated with a perturbation of the potential $\tilde{V}$. In other words

$$
\delta L=\left(\begin{array}{c|c}
0 & 0 \\
\hline \delta \tilde{V} & 0
\end{array}\right)
$$

whose operator norm induced by the energy norm is given (cf. equation (B.15) in [53]) by

$$
\|\delta L\|_{E}=\left(\rho\left((\delta L)^{\dagger} \delta L\right)\right)^{\frac{1}{2}}=\max \left\{\sqrt{\lambda} \mid \lambda \in \sigma\left((\delta L)^{\dagger} \delta L\right)\right\}
$$

where $\dagger$ represents the adjoint respect to the inner product $\langle\cdot, \cdot\rangle_{E}$. Although $L$ and $\delta L$, are in general differential operators, in the current heuristic assessment we will make use of discrete approximates, to cast the discussion into matricial terms (a proper differential operator treatment is needed and will be developed elsewhere). Let $[L]$ and $[\delta L]$ denote the discrete version of $L$ and $\delta L$, respectively, and let $[L]$ and $[\delta L] \in \mathbb{C}_{2 n, 2 n}$. In this discrete setup, the scalar product can be encoded in the Gram matrix $\left[G^{E}\right] \in \mathbb{C}_{2 n, 2 n}$ and the adjoint operator is given by $[\delta L]^{\dagger}=\left[G^{E}\right]^{-1} \cdot[\delta L]^{*} \cdot\left[G^{E}\right]$ where the expression is understood in the matricial sense and the symbol ${ }^{*}$ denotes again the standard transposition-conjugation operation for matrices. Let the Gram matrix and its inverse be schematically represented as $\$$

$$
\left[G^{E}\right]^{-1}=\left(\begin{array}{c|c}
G_{A} & G_{B} \\
\hline G_{C} & G_{D}
\end{array}\right) \quad, \quad\left[G^{E}\right]=\left(\begin{array}{c|c}
G^{A} & G^{B} \\
\hline G^{C} & G^{D}
\end{array}\right) .
$$

Using this notation, a direct calculation renders

$$
[\delta L]^{\dagger}[\delta L]=\left(\begin{array}{l|l}
G_{A}[\delta \tilde{V}]^{*} G^{D}[\delta \tilde{V}] & 0 \\
\hline G_{C}[\delta \tilde{V}]^{*} G^{D}[\delta \tilde{V}] & 0
\end{array}\right) .
$$

For the upcoming discussion, we note that given a block matrix $M$ of the form

$$
M:=\left(\begin{array}{c|c}
M_{A} & M_{B} \\
\hline M_{C} & M_{D}
\end{array}\right),
$$

one can exploit the Schur's complement of the matrix (198) under the assumption that $M_{D}$ is invertible — see [111] — to express the determinant of $M$ in terms of the block matrices as

$$
\operatorname{det} M=\operatorname{det} M_{D} \operatorname{det}\left(M_{A}-M_{B}\left(M_{D}\right)^{-1} M_{C}\right) \text {. }
$$

In order to address the estimation of the norm $\|\delta L\|_{E}$ given by equation (195), we set

$$
\begin{array}{ll}
M_{A}=G_{A}[\delta \tilde{V}]^{*} G^{D}[\delta \tilde{V}]-\lambda I, & M_{B}=0, \\
M_{C}=G_{C}[\delta \tilde{V}]^{*} G^{D}[\delta \tilde{V}], & M_{D}=-\lambda I,
\end{array}
$$

where $I$ is the identity matrix of size $n$. Substituting then equations 200] in [199], one obtains

$$
\operatorname{det}\left([\delta L]^{\dagger}[\delta L]-\lambda I\right)=(-\lambda)^{n} \operatorname{det}\left(G_{A}[\delta \tilde{V}]^{*} G^{D}[\delta \tilde{V}]-\lambda I\right)
$$

$\ddagger$ Observe that although in general $\left(G^{\mathbf{i}}\right)^{-1} \neq G_{\mathbf{i}}$, - here $\mathbf{i}$ are label-indices with $\mathbf{i}=A, B, C, D$ - the relation between $G_{\mathbf{i}}$ and $G^{\mathbf{i}}$ can be found explicitly under suitable assumptions using Schur's complement method for the treatment of block matrices — see [111]. These relations will not be needed for the subsequent discussion. 
Observe that the assumption that $M_{D}$ is invertible is equivalent in the present case to $\lambda \neq 0$. Thus, as long as $0 \notin \sigma\left([\delta L]^{\dagger}[\delta L]\right)$ one has that

$$
\sigma\left([\delta L]^{\dagger}[\delta L]\right)=\sigma\left(G_{A}[\delta \tilde{V}]^{*} G^{D}[\delta \tilde{V}]\right)
$$

The discussion up to this point has been independent of the form of the matrix $[\delta \tilde{V}]$ and the chosen Gram matrix, with the only assumptions $0 \notin \sigma\left([\delta L]^{\dagger}[\delta L]\right)$. We impose now $[\delta \tilde{V}]$ to be a diagonal matrix, as it corresponds to a perturbation of the potential $\tilde{V}$, namely

$$
[\delta \tilde{V}]=\operatorname{diag}\left(\delta \tilde{V}_{1}, \ldots, \delta \tilde{V}_{n}\right),
$$

with $\delta \tilde{V}_{i}, i \in\{1, \ldots, n\}$ the discretized values of the function $\delta \tilde{V}$ perturbing $\tilde{V}$.

Before applying the previous discussion to the Gram matrix associated with the energy scalar inner product [101, it is illustrative to consider the simpler case of the standard $L^{2}$ scalar product for which the Gram matrix reduces to the identity; $\left[G_{2}\right]=I_{2 n}$. In other words, $G_{A}=G^{A}=G_{D}=G^{D}=I_{n}$ and $G_{B}=G^{B}=G_{C}=G^{C}=0$. Then, using equation 202, along with (203) and (195) one concludes that

$$
\|\delta L\|_{2}=\max \left\{\left|\delta \tilde{V}_{i}\right|, i \in\{1, \ldots, n\}\right\},
$$

that is, $\|\delta L\|_{2}$ is given by the largest (in absolute value) of the discretised values of the potential perturbation $\delta \tilde{V}$. This discretised reasoning leads to the point-like expression

$$
\|\delta L\|_{E}=\max _{\operatorname{supp}(\delta \tilde{V})}|\delta \tilde{V}|
$$

for the norm of $\|\delta L\|_{E}$, where $\operatorname{supp}(\delta \tilde{V})$ is the support of the perturbation $\delta \tilde{V}$. We conclude that large perturbations of the $L$ operator are associated with "peaked" distributions of the perturbation of the potential. This conclusion, with "modulations" in the case of the energy norm (see below), is the main message to retain from this discussion.

For the energy scalar product, observe that equation (101) can be written as

$$
\left\langle u_{1}, u_{2}\right\rangle_{E}=\int_{a}^{b}\left(\bar{\phi}_{1}, \bar{\psi}_{1}\right)\left(\begin{array}{c|c}
-\partial_{x}\left(p \partial_{x}\right)+\tilde{V} & 0 \\
\hline 0 & w
\end{array}\right)\left(\begin{array}{c}
\phi_{2} \\
\psi_{2}
\end{array}\right) d x .
$$

where $u=(\phi, \psi)$, we have used integration by parts and exploited that $p(a)=p(b)=0$. Thus, one can make the following identifications $G_{B}=G^{B}=G_{C}=G^{C}=0$ and

$$
\begin{array}{lll}
G^{D}=w I, & G^{A}=-\partial_{x}\left(p \partial_{x}\right)+\tilde{V} \\
G_{D}=w^{-1} I, & G_{A}=\left(-\partial_{x}\left(p \partial_{x}\right)+\tilde{V}\right)^{-1} .
\end{array}
$$

Hence using equations (202), 203, and 206 renders

$$
\sigma\left([\delta L]^{\dagger}[\delta L]\right)=\sigma\left(\left(-\partial_{x}\left(p \partial_{x}\right)+\tilde{V}\right)^{-1} w|\delta \tilde{V}|^{2}\right) .
$$

Using definition of the operator $L_{1}$ in equations (3) (or (100) taking into account the notation $q=\tilde{V})$, one has that $L_{1}^{-1}=\left(\partial_{x}\left(p \partial_{x}\right)-\tilde{V}\right)^{-1} w$ and thus, one can rewrite equation (207) as

$$
\sigma\left([\delta L]^{\dagger}[\delta L]\right)=\sigma\left(-L_{1}^{-1}|\delta \tilde{V}|^{2}\right) .
$$

This last expression can be read as a relation between the spectrum of operators rather than matrices. Thus, using expression (195), we have

$$
\|\delta L\|_{E}^{2}=\max \left\{|\lambda|, \lambda \in \sigma\left(L_{1}^{-1}|\delta \tilde{V}|^{2}\right)\right\} .
$$


An estimate of this quantity, appropriate in the setting of the present heuristic discussion, is given by the notion of "numerical abscisa" $\omega(A)$ of an operator $A$, defined as the supremum of the real part of its numerical range $W(A)$ and that can be characterised [105] as

$$
\omega(A)=\sup \sigma\left(\frac{1}{2}\left(A+A^{\dagger}\right)\right) .
$$

Since $\frac{1}{2}\left(A+A^{\dagger}\right)$ is selfadjoint, we can use Rayleigh-Ritz theorem (e.g. [94, 7]) to characterize $\S$

$$
\omega(A)=\sup _{\|u\|=1}\{\langle u, A u\rangle\} .
$$

In our case, although $L_{1}$-and hence also $L_{1}^{-1}-$ and $|\delta \tilde{V}|^{2}$ are selfadjoint operators, the composite operator $L_{1}^{-1}|\delta \tilde{V}|^{2}$ is not, since

$$
\left(L_{1}^{-1}|\delta \tilde{V}|^{2}\right)^{\dagger}=\left(|\delta \tilde{V}|^{2}\right)^{\dagger}\left(L_{1}^{-1}\right)^{\dagger}=|\delta \tilde{V}|^{2} L_{1}^{-1} \neq L_{1}^{-1}|\delta \tilde{V}|^{2} .
$$

We can put together the previous discussion to estimate $\|\delta L\|_{E}^{2}$ in equation 209] as

$$
\begin{aligned}
\|\delta L\|_{E}^{2} & \sim \sup \sigma\left(\frac{1}{2}\left(L_{1}^{-1}|\delta \tilde{V}|^{2}+\left(L_{1}^{-1}|\delta \tilde{V}|^{2}\right)^{\dagger}\right)\right) \\
& =\sup _{\|u\|=1}\left\{\left\langle u, \frac{1}{2}\left(L_{1}^{-1}|\delta \tilde{V}|^{2}+|\delta \tilde{V}|^{2} L_{1}^{-1}|\delta \tilde{V}|^{2}\right) u\right\rangle\right\}
\end{aligned}
$$

Exploiting the fact that $L_{1}$ is a Sturm-Liouville operator, namely, selfadjoint in the space $\left(L^{2}, w(x) d x\right)$, one can express its inverse (adopting a usual bra-ket notation) as

$$
L_{1}^{-1}=\sum_{n} \frac{1}{\lambda_{n}}\left|\ell_{n}\right\rangle\left\langle\ell_{n}\right|,
$$

where $\lambda_{n}^{1}$, and $\left|\ell_{n}\right\rangle$ denote now respectively, the eigenvalues and normalized eigenvectors of $L_{1}$, using that the former constitute an orthonormal basis for the function space where $L_{1}$ is defined. In order to justify this expression, in this discussion $L_{1}$ is thought as an operator defined on the compact, 3-dimensional manifold corresponding to the compactified hyperboloid $\Sigma$ with regularity imposed on the corresponding cut of null infinity $\Sigma \cap \mathscr{I}^{+}$. This guarantees the discrete character of the spectrum and, for generic potentials, the trivial kernel of $L_{1}$ and therefore the absence of zero eigenvalues. Therefore expression 214 makes sense.

Similarly, for the operator $\delta \tilde{V}$ we can write

$$
|\delta \tilde{V}|^{2}=\int d x|\delta \tilde{V}|^{2}|x\rangle\langle x|,
$$

where $|x\rangle$ denotes the eigenvectors of the position operator $X$, namely $X|x\rangle=x|x\rangle$. Using expansions (214) and 215) to estimate the right hand side of equation 213, we get

$$
\begin{aligned}
\left.2|| \delta L\right|_{E} ^{2} & \sim\left\langle u \mid \sum_{n} \frac{1}{\lambda_{n}} \ell_{n}\right\rangle\left\langle\left.\ell_{n}\left|\int d x\right| \delta V\right|^{2} \mid x\right\rangle\langle x \mid u\rangle+\left\langle\left. u\left|\int d x\right| \delta V\right|^{2} \mid x\right\rangle\left\langle x \mid \sum_{n} \frac{1}{\lambda_{n}} \ell_{n}\right\rangle\left\langle\ell_{n} \mid u\right\rangle \\
& \lesssim \frac{\sup |\delta \tilde{V}|^{2}}{\min \lambda_{n}}\left\langle u \mid \sum_{n} \ell_{n}\right\rangle\left\langle\ell_{n}\left|\int d x\right| x\right\rangle\langle x \mid u\rangle+\frac{\sup |\delta \tilde{V}|^{2}}{\min \lambda_{n}}\left\langle u\left|\int d x\right| x\right\rangle\left\langle x \mid \sum_{n} \ell_{n}\right\rangle\left\langle\ell_{n} \mid u\right\rangle \\
& =2 \frac{\sup |\delta \tilde{V}|^{2}}{\inf \lambda_{n}}\langle u \mid u\rangle=2 \frac{\sup |\delta \tilde{V}|^{2}}{\inf \lambda_{n}},
\end{aligned}
$$

$\S$ We could have just written this from the starting definition of the numerical abscissa $\omega(A)$ in terms if the numerical range of $A$, namely $W(A)=\{\langle u, A u\rangle,\|u\|=1\}$, but we have preferred to pass through the Rayleigh-Ritz, familiar for physicists in the quantum setting. See [105] for details and properties of numerical range and abscissa. 
where we have used the expression of the identity operator in the Hilbert bases $\left\{\left|\ell_{n}\right\rangle\right\}$ and $\{|x\rangle\}$ and, in the last line, the normalization $\|u\|=1$. Finally, using the order of magnitude estimate inf $\lambda_{n} \sim \max _{\operatorname{supp}(\tilde{V})} \tilde{V}>0$, one concludes that $\|$

$$
\|\delta L\|_{E} \sim \alpha \max _{\operatorname{supp}(\delta \tilde{V})}|\delta \tilde{V}| \quad, \quad \alpha=\frac{1}{\left(\max _{\operatorname{supp}(\tilde{V})} \tilde{V}\right)^{\frac{1}{2}}}
$$

Contrast this result with the estimation in equation (204) from the standard $L^{2}$ scalar product: we still have an estimation such that large norms $\|\delta L\|_{E}$ correspond to "peaked" distributions of the perturbation potential $\delta \tilde{V}$, but the estimation is not really pointwise, but rather nonlocal, since maxima of $\delta \tilde{V}$ and $\tilde{V}$ occur generically at different locations.

Finally, to make contact with spacetime perturbations $\delta g_{a b}$, we consider $\epsilon$-sized perturbations $\delta L$ (i.e. $\|\delta L\|_{E}=\epsilon$ ), so putting together 217) with the estimation (191) for $\delta \tilde{V}$

$$
\epsilon \sim \alpha \max _{\operatorname{supp}(\delta g)}\left|\partial^{2}\left(\delta g_{a b}\right)\right|
$$

that, combined with 193, relates $\epsilon$ to the density of energy of the spacetime perturbation

$$
\epsilon \delta g \sim \alpha \max _{\operatorname{supp}(\delta g)}\left|\partial\left(\delta g_{a b}\right)\right|^{2} \sim \alpha \max _{\operatorname{supp}(\delta g)} \rho_{E}(\delta g ; x)
$$

Therefore, although modulated by a factor $\alpha$-consequence of the energy-inner product $\langle\cdot, \cdot\rangle_{E}$ and controlled by the "curvature maximum" of the non-perturbed geometryspacetime perturbations involving larger $\epsilon$ perturbations of the operator $L$ (and therefore triggering stronger QNM instabilities of the overtones), are associated with strong peaks in the "energy distribution" of the perturbation, rather than to the integrated energy distribution: to efficiently perturb with a given available energy, use peaked distributions of perturbations.

\subsection{High-frequency limit of general relativity and spacetime regularity: Burnett's conjecture and QNM high-frequency perturbations}

We have closed section 5.1.3 and the discussion of the emerging picture of BH QNM instability in section 5.2 by posing the question about the possible universality of $\mathrm{BH}$ perturbed QNM branches asymptotics, in particular the relation to pseudospectra boundaries, when the perturbation frequency tends to infinity. In this context, the precedent section 6.1 provides an additional key ingredient, namely the fact that perturbations with a "spiked structure" are more efficient in triggering BH QNM overtone instabilities. This points towards a low-regularity phenomenon underlying the genericity of perturbed BH QNM asymptotics.

In addition, as commented in section 5.2, perturbed BH QNM branches resulting from high-frequency perturbations share some qualitative features with QNMs of matter compact objects, namely with so-called $w$-modes or curvature modes of neutron stars. In this setting relating matter and high-frequency vacuum spacetimes, it may be of interest to bring attention to the so-called Burnett's conjecture [17], namely stating that in the limit of infinite highfrequency a vacuum spacetime can be understood in effective terms as a spacetime filled with matter, namely with a stress-energy tensor given by Vlasov massless matter.

The high-frequency limit of general relativity has been the subject of systematic studies [46, 47, 21, 22, 75], in particular aiming at elucidating the properties of rapidly

$\|$ Note that the expression is consistent from the perspective of physical dimensions, since the operator $L$ (and therefore $\delta L$ ) has the physical dimensions of a frequency, as follows from equation (4). 
oscillating gravitational wave spacetimes. Burnett's approach considers a sequence of vacuum spacetimes $\left\{\left(M, g_{n}\right)\right\}_{n=1}^{\infty}$ (we follow the presentation in [74]) satisfying $R_{a b}=0$, (weakly) converging to $g_{\infty}$ in the limit $n \rightarrow \infty$. However, the resulting metric $g_{\infty}=\lim _{n \rightarrow \infty} g_{n}$ is generically not a vacuum spacetime, but satisfies $R_{a b}-\frac{1}{2} R g_{a b}=8 \pi T_{a b}$ for an appropriate stress-energy tensor. For this result, specific conditions are required on $\left\{\left(M, g_{n}\right)\right\}_{n=1}^{\infty}$ that encode the desired properties of the high-frequency limit, in particular the vanishing of perturbations amplitudes as the frequency goes to infinity (see [17] for details). Specifically, it assumes the existence of constants $C$ and $\lambda_{n}$ (with $\lim _{n \rightarrow \infty} \lambda_{n}=0$ ) such that

$$
\left|g_{n}-g_{\infty}\right| \leq \lambda_{n},\left|\partial g_{n}\right| \leq C,\left|\partial^{2} g_{n}\right| \leq C \lambda_{n}^{-1}
$$

Under these conditions, and building on a theorem on the convergence of the sequence $\left\{\left(M, g_{n}\right)\right\}_{n=1}^{\infty}$, Burnett conjectures that the limiting metric $g_{\infty}$ is isometric to a solution of the massless Vlasov-Einstein system, namely

$$
G_{a b}=8 \pi T_{a b}, \nabla_{a} T^{a b}=0
$$

with

$$
T_{a b}=\int a^{2}(x, k) k_{a} k_{b} d V_{k}, \quad k^{c} \nabla_{c} a^{2}(x, k)=0,
$$

where $a^{2}$ is a "particle" distribution function in the cotangent bundle $T^{*} M$, with $k_{a}$ constrained to be null $\left(k^{a} k_{a}=0\right)$ and, therefore, the integration - with measure $d V_{k}$ - is performed on the null cone in the cotangent space $T_{x}^{*} M$ (at each $x \in M$ ).

Although no proof is available in the fully general case, Burnett's conjecture has been proved under the further condition of $\mathbb{U}(1)$ symmetry [44] (see also [43, 42]). Burnett's conjecture captures the idea that a vacuum spacetime, under (gravitational wave) highfrequency perturbations, behaves effectively as a matter system, a tantalizing picture when comparing perturbed BH QNM overtones with $w$-modes in matter compact stars.

Although Burnett's conditions 220) allow for infinitely rapid oscillations, they do not permit "concentration" of the oscillations. However, as seen in section 6.1. concentration of perturbations is a crucial element in the analysis of BH QNM perturbations. Such capability to deal with concentrations (in addition to oscillations) requires the relaxation of conditions 220. This has been addressed by Luk \& Rodnianski in their study of "null shells" in the more general setting of low-regularity problems of Einstein equations [74] (cf. also [72, 73] and references therein). In particular, by relaxing convergence conditions of $\left\{\left(M, g_{n}\right)\right\}_{n=1}^{\infty}$ to

$$
g_{n} \rightarrow g_{\infty} \text { in } C^{0}, \partial g_{n} \rightarrow \partial g_{\infty} \text { in } L^{2},
$$

Luk \& Rodnianski have proven [73] that the limiting metric is $C^{0} \cap H^{1}$ (with $H^{1}=W^{1,2}$ the Sobolev space controlling the energy, precisely the kind of norm in our QNM instability assessment) and, appropriately formulated, it satisfies Einstein equation [74] in an Einsteinnull dust system. This provides a resolution of a relaxed version of Burnett's conjecture.

The key point for the assessment of BH QNM asymptotics is that the limit of infinite high-frequency oscillations allowing from "perturbation concentrations" is a "low-regularity" limit, in particular the limiting metric is generically in the $C^{0}$ class. Putting this together with our discussion of Regge QNMs for $C^{p}$ potentials in section 5.1.3, the involved low-regularity limit directly leads to refining the conjecture in [54] about the logarithmic asymptotics of BH QNM branches in the infinite high-frequency limit under generic ultraviolet oscillations.

6.2.1. Ultraviolet QNM perturbations: Regge QNM branches conjecture. Let us recall the behaviour of perturbed BH QNM overtones under perturbations of increasing frequency. As 
described in section 5.2, increasing the frequency of the perturbations push the perturbed $\mathrm{BH}$ QNMs towards the logarithm pseudospectrum lines. Such perturbed QNMs stay strictly above the logarithmic lines as long as the frequency is high but finite: there is no guarantee that the logarithmic lines are attained in the high-frequency limit and, in case QNMs indeed reach the pseudospectrum boundary in this a limit, no information about the specific distribution of QNMs along such logarithmic lines is available. On the the other hand, for the instance of "infinite frequency" provided by $C^{p}$ potentials, the resulting Regge QNM branches are indeed logarithmic branches with a very specific distribution of QNMs given by expressions (174).

Putting together the $C^{p}$ potential case with Luk \& Rodnianski version of Burnett's conjecture allowing for oscillations and concentration, it seems natural to expect that Regge QNMs are not just an instance but they describe the generic infinite high-frequency limit of all perturbations. Specifically, we propose the following Regge QNM conjecture:

In the limit of infinite frequency, generic ultraviolet perturbations push BH QNMs in Nollert-Price branches to asymptotically logarithmic branches along the boundary of the QNM-free region, following precisely the Regge QNM asymptotic pattern in (174).

\subsection{Towards a geometric description of QNMs}

Following [53], our discussion of BH QNM instability explicitly relies on a formulation of the hyperboloidal approach to QNMs depending on an appropriate choice of coordinates, in particular tied to spherical symmetry. However, the discussions in the previous sections strongly suggest the naturality of promoting the whole discussion to a genuine geometric formulation, in arbitrary dimensions and to generic spacetimes without symmetries T. This section is simply meant as a heuristic exploration, putting in order some of the elements to be incorporated in a systematic geometric formulation to be discussed in a future work [37].

Arguably, the only crucial features in the construction are i) that the spacetime foliation intersects null-infinity and the BH horizon at, respectively, cuts $\mathcal{S}_{\tau}^{\infty}$ and $\mathcal{S}_{\tau}^{\mathcal{H}}$ and ii) that the key analytic structures admit a lifting to a geometric set up, where the coordinates and particular foliation do not play a fundamental role. Methodologically, we focus on a hyperboloidal approach, even if this is not the only option $\square$ We list the main steps in the construction:

1. Compactified hyperboloidal slicing: geometric data. Spacetime is described in a compactified hyperboloidal slicing $\left\{\Sigma_{\tau}\right\}$, where slices are compactified manifolds with boundary. Boundaries $\mathcal{S}_{\tau}^{\infty}$ correspond to the intersection of $\Sigma_{\tau}$ with future null infinity (together with horizon slices $\mathcal{S}_{\tau}^{\mathcal{H}}$ in $\mathrm{BH}$ spacetimes). Data are given by: i) the slicings $\left\{\Sigma_{\tau}\right\},\left\{\mathcal{S}_{\tau}^{\infty}\right\}$ (and $\left\{\mathcal{S}_{\tau}^{\mathcal{H}}\right\}$ in BH spacetimes), ii) the intrinsic and extrinsic geometries of $\Sigma_{\tau}$ (respectively $\gamma_{a b}$ and $K_{a b}$, possibly including an effective potential $V$ ), iii) a choice of transverse null normals $k^{a}$ at $\mathscr{I}^{+}$, normal to $\{\mathcal{S}\}_{\tau}^{\infty}$ and the null generator $\ell^{a}$ at $\mathscr{I}^{+}$ (analogously at the horizon $\mathcal{H}$ ), iv) a function $\gamma$ on $\{\mathcal{S}\}_{\tau}^{\infty}$ (similarly $\{\mathcal{S}\}_{\tau}^{\mathcal{H}}$ ), and iv) an integration measure $d \mu=w(x) d \Sigma_{\tau}$ on $\Sigma_{\tau}$. Constraints exist among these data.

2. Geometric formulation of dynamics and scalar product. A first order formulation of the dynamics is formulated in terms of an evolution operator $L$ constructed from operators $L_{1}$ and $L_{2}$ on $\Sigma_{\tau}$. $L_{1}$ is elliptic and depending only on the intrinsic geometry (and on $V$ ) of $\Sigma_{\tau}$, whereas $L_{2}$ is a first order operator depending on the extrinsic geometry. A scalar product is built from the data, $L_{2}$ accounting for the loss of selfadjointness.

3. A geometric approach to QNM characterization. QNMs are proposed to arise from an eigenvalue problem for $L$. Outgoing boundary conditions are encoded in $L_{2}$ and a

I We acknowledge A. Ashtekar for stressing the relevance and need of addressing this geometric aspect.

+ Null slicings of spacetime could also be considered here. This will be addressed elsewhere. 
geometric condition of regularity for eigenfunctions, namely enforcing $L_{1}$ to "live" on a smooth closed (compact without boundary) version $\Sigma_{\tau}^{\circ}$ of $\Sigma_{\tau}$. In particular, smoothness of $\Sigma_{\tau}^{\circ}$ would entail the elimination of the continuous ("branch cut") spectrum of $L$.

4. Towards a notion of normal modes for isolated systems: additional degrees of freedom and BMS symmetry. Lack of selfadjointness in our scattering problem follows from the flow of degrees of freedom through null infinity (and the horizon). Restituting selfadjointness requires enlarging the system to account for the loss of such degrees of freedom at the boundaries, so that the full system is conservative. We claim that such degrees of freedom can be encoded in terms of the asymptotic (dynamical) BMS symmetry at null infinity (and an analogous structure at the horizon). Resulting eigenvalues would provide a notion of (real) normal modes of isolated object spacetimes.

In the rest of this section, we provide some further details in the scheme above, having the scattering of a scalar field as the main reference problem in mind.

6.3.1. Compactified hyperboloidal slicing: geometric data. Let us start with a stationary (with Killing vector $t^{a}$ ) asymptotically simple physical spacetime $(\mathcal{M}, g)$, with null infinity $\mathscr{I}^{+}$and a horizon $\mathcal{H}$, in case of dealing with a BH spacetime. Topology of $\mathscr{I}^{+}$and $\mathcal{H}$ are $\mathbb{S}^{2} \times \mathbb{R}$. Let us consider a conformal extension of the physical spacetime $(\tilde{\mathcal{M}}, \tilde{g})$. Let us denote by $\ell^{a}$ the null generators along $\mathscr{I}^{+}$and $\mathcal{H}$. We proceed then in the following stages:

i) Start by choosing a slicing $\left\{\mathcal{S}_{\tau}^{\infty}\right\}$ of $\mathscr{I}^{+}$. Given the generator $\ell^{a}$ along $\mathscr{I}^{+}$consider, at each slice $\mathcal{S}_{\tau}^{\infty}$, the only null normal $k^{a}$ to $\mathcal{S}_{\tau}^{\infty}$ satisfying $\ell^{a} k_{a}=-1$. In a BH spacetime, repeat the procedure at the $\mathrm{BH}$ (Killing) horizon $\mathcal{H}$, i.e. choose a slicing $\left\{\mathcal{S}_{\tau}^{\mathcal{H}}\right\}$ fixing the associated transverse $k^{a}$ null normal, $\ell^{a} k_{a}=-1$, at horizon sections $\mathcal{S}_{\tau}^{\mathcal{H}}$.

ii) Choose a function $\gamma$ on null infinity sections $\mathcal{S}_{\tau}^{\infty}$ (respectively, also a function $\gamma$ on $\mathcal{S}_{\tau}^{\mathcal{H}}$ in BH spacetimes) and consider the null vector $\gamma^{a}=\gamma k^{a}$, outgoing from $\mathcal{M}$.

iii) Extend the vector $\gamma^{a}$ arbitrarily to the bulk, subject to the following constraints: a) $\gamma^{a}$ is tangent to a spacelike slicing $\left\{\Sigma_{\tau}\right\}$ of $\mathcal{M}$ adapted to stationary (namely, the Killing vector $t^{a}$ transports slices of $\left\{\Sigma_{\tau}\right\}$ into slices of $\left\{\Sigma_{\tau}\right\}$ ), b) $\gamma^{a}$ is spacelike everywhere, except at the boundaries, where it is null. The slicing $\left\{\Sigma_{\tau}\right\}$ is a hyperboloidal one.

iv Slices $\Sigma_{\tau}$ in $\tilde{\mathcal{M}}$ are compact manifolds with boundary, with boundaries given by spheres. Let us consider the intrinsic and extrinsic geometry of $\Sigma_{\tau}$, namely the induced metric $\gamma_{a b}$ and the extrinsic curvature $K_{a b}=\mathcal{L}_{n} \gamma_{a b}$, with $n^{a}$ the unit timelike normal to $\Sigma_{\tau}$. Let us introduce a measure $d \mu=w d \Sigma_{\tau}$, with $w$ a weight function on $\Sigma_{\tau}$.

v) The resulting data ${ }^{*}$ in the problem are: $\left(\left\{\Sigma_{\tau}\right\}, \gamma_{a b}, K_{a b}, w ;\left\{\mathcal{S}_{\tau}^{\infty}\right\},\left\{\mathcal{S}_{\tau}^{\mathcal{H}}\right\}, \gamma\right)$.

6.3.2. Geometric formulation of dynamics and scalar product. One of the most interesting results in [53], is the identification of analytic structure of operators $L_{1}$ and $L_{2}$, building the evolution generator $L$, as appearing in equation (3). In particular, the singular Sturm-Liouville structure of $L_{1}$ is closely related to a Laplacian-like operator, whereas the structure of $L_{2}$ also calls for a natural geometric generalization in terms of a vector field $\gamma^{a}$.

\footnotetext{
* These data are redundant, namely they admit constraints. They ultimately should be reduced just to the choice of slicings of the boundaries smoothly extending to the bulk in a hyperboloidal slicing. Given the slicings $\left\{\mathcal{S}_{\tau}^{\infty}\right\}$ of $\mathscr{I}+$ and $\left\{\mathcal{S}_{\tau}^{\mathcal{H}}\right\}$ of $\mathcal{H}$, extend and join them by a a hyperboloidal slicing $\left\{\Sigma_{\tau}\right\}$. Denote the induced metric on $\Sigma_{\tau}$ by $\gamma_{a b}$ and the extrinsic metric by $K_{a b}=\mathcal{L}_{n} \gamma_{a b}$. Then, defining $\gamma=K_{a b} \ell^{a} k^{a}$ at the boundaries, consider the outgoing null vector at the boundaries $\gamma^{a}=\gamma k^{a}$. Considering the timelike vector $t^{a}$, we can decompose it as $t^{a}=N n^{a}+\beta^{a}$, in terms of lapse function $N$ and shift vector $\beta^{a}$. Then $K_{a b}=\frac{1}{2 N}\left(\partial_{t} \gamma_{a b}+D_{a} \beta_{b}+D_{b} \beta_{a}\right)=$ $\frac{1}{2 N}\left(D_{a} \beta_{b}+D_{b} \beta_{a}\right)$. Also, note $\sqrt{g} d^{n} x=N d \tau d \Sigma_{t}$.
} 
For concreteness, denoting by $\phi$ the degrees of freedom whose hyperbolic dynamics we are considering, we write their first-order (in time) evolution in terms of data above as

$$
\partial_{\tau} u=i L u, u=\left(\begin{array}{c}
\phi \\
\psi=\partial_{\tau} \phi
\end{array}\right), L=\frac{1}{i}\left(\begin{array}{c|c}
0 & 1 \\
\hline L_{1} & L_{2}
\end{array}\right)
$$

where

$$
L_{1}=\frac{1}{w}(\tilde{\Delta}-\tilde{V}(x)), \quad L_{2}=\frac{1}{w}(2 \gamma \cdot \tilde{D}+\tilde{D} \cdot \gamma)
$$

Here $L_{1}$ and $L_{2}$ are operators living on $\Sigma_{\tau}$ (with boundaries), $\tilde{\Delta}$ denotes the Laplacian with respect to the compactified metric $\tilde{\gamma}_{a b}$, namely $\tilde{\Delta}=\tilde{\gamma}^{a b} \tilde{D}_{a} \tilde{D}_{b}$, and $\tilde{V}$ is a possible effective potential, depending on the nature of the dynamical field $\phi$.

The scalar product is then proposed to be

$$
\left\langle u_{1}, u_{2}\right\rangle_{E}=\frac{1}{2} \int_{\Sigma_{\tau}}\left(w \bar{\psi}_{1} \psi_{2}+\tilde{D} \bar{\phi}_{1} \cdot \tilde{D} \phi_{2}+\tilde{V} \bar{\phi}_{1} \phi_{2}\right) d \tilde{\Sigma},
$$

where the $\cdot$ scalar product is calculated with respect to $\tilde{\gamma}_{a b}$ and $d \tilde{\Sigma}$ denotes the volume element in $\left(\Sigma_{\tau}, \tilde{\gamma}_{a b}\right)$. Then, the formal adjoint operator would be

$$
L^{\dagger}=L+L^{\partial} \quad, \quad L^{\partial}=\frac{1}{i}\left(\begin{array}{c|c}
0 & 0 \\
\hline 0 & L_{2}^{\partial}
\end{array}\right),
$$

with $L_{2}^{\partial}$ given by the expression

$$
L_{2}^{\partial}=-2 \frac{\gamma^{a} \ell_{a}}{w}\left(\delta_{S^{\mathcal{H}}}-\delta_{S^{\infty}}\right)=-2 \frac{\left(\gamma \ell^{a}\right) k_{a}}{w}\left(\delta_{S^{\mathcal{H}}}-\delta_{S^{\infty}}\right)
$$

where $\gamma^{a} \ell_{a}=\gamma k^{a} \ell_{a}=-\gamma$. The reason of rewriting it as $\left(\gamma \ell^{a}\right) k_{a}$, with $\ell^{a}$ the generator of the null boundaries, will be apparent below.

In sum, expressions in this subsection provide a (purely formal) geometric extension of the relevant equations in [53], upon the choice of the boundary foliations.

6.3.3. A geometric approach to QNM characterization. Taking Fourier transform in time of equation (224), we obtain the eigenvalue problem characterization QNMs. Outgoing boundary conditions are enforced by the geometric frame, given that the boundaries are null hypersurfaces and the light cones point outwards. However, such boundary conditions must be supplemented with appropriate regularity conditions on the eigenfunctions. This defines a challenging problem in analysis, namely the identification of the appropriate functional space of eigenfunctions [110, 33, 34, 35]. In particular, the treatment in [53] suffers from the severe problem that the spectrum of $L$ contains a continuous part.

The approach we propose here is to define QNMs in a "radically" geometric manner, by imposing $L_{1}$ to be defined/regularized in a closed manifold $\Sigma_{\tau}^{\circ}$. This would be obtained by:

i) First, shrinking the $\mathbb{S}^{2}$ boundaries of $\Sigma_{\tau}$ to respective points $i_{\infty}$ and $i_{\mathcal{S}}$. The manifold $\Sigma_{\tau}$ is then a 3-sphere $\mathbb{S}^{3}$ with two pinched-holes corresponding to its boundaries.

ii) Second, the operator $L_{1}$ is regularized to an operator $\stackrel{\circ}{L}_{1}$, so that it is well defined on a full smooth 3 -sphere $\mathbb{S}^{3}$ obtained by adding "two points" to complete $\Sigma_{\tau}$.

Certainly, problems will appear in general when trying to obtain a regular $\stackrel{\circ}{L}_{1}$. For instance, whereas the problem seems straightforward in the Pöschl-Teller case, in Schwarzschild one should expect obstructions due to the non-vanishing mass, leading to the existing branches. Regularizing the operator on a smooth 3 -sphere can then be seen as a tantamount of "cutting" 
the continuous part of the spectrum, leaving only eigenvalues representing the "geometric counterpart" of the identification of the proper functional space in the analytical approach. Assuming this can be achieved, QNMs are defined from the (pure)-eigenvalue problem

$$
\left(\begin{array}{c|c}
0 & 1 \\
\hline L_{1} & L_{2}
\end{array}\right)\left(\begin{array}{c}
\phi_{n} \\
\psi_{n}
\end{array}\right)=\omega_{n}\left(\begin{array}{c}
\phi_{n} \\
\psi_{n}
\end{array}\right),
$$

where $\stackrel{\circ}{L}_{1}$ lives on a smooth $\mathbb{S}^{3}$, whereas $L_{2}$ lives on a "pinched" $\mathbb{S}^{3}$, with holes at $i_{\infty}$ and $i_{\mathcal{S}}$ where the outgoing "degrees of freedom" are encoded. Note that, given the absence of boundaries, the scalar product 226) can then be written in terms of the $L_{1}$ as

$$
\left\langle u_{1}, u_{2}\right\rangle_{E}=\frac{1}{2} \int_{\Sigma_{\tau}}\left(w \bar{\psi}_{1} \psi_{2}-\bar{\phi}_{1} \stackrel{\circ}{L}_{1} \phi_{2}\right) d \tilde{\Sigma}
$$

Note that $L_{2}$ plays no role in this (energy) scalar product.

6.3.4. Missing degrees of freedom and BMS symmetry: towards normal modes in isolated objects. Non-selfadjointness in dynamical problems generically reflects that some degrees of freedom are missing or are being lost: the system is not isolated or is part of a larger system, leading to non-conservative dynamics. In order to cast such systems in terms of a selfadjoint problem, one must "complete" the system by adding an appropriate set of degrees of freedom. In our setting, how could we render our BH perturbation problem selfadjoint?

The compactified picture provides a geometric frame to address such a question. In our scattering case, degrees of freedom flow to infinity and through the back hole horizon. If we consider adding formal degrees of freedom at future null infinity $\mathscr{I}^{+}$and at the BH horizon $\mathcal{H}$, in such a way that they account for the degrees of freedom and energy leaving the system, then we could consider formulating the dynamics in an enlarged system where energy is actually conserved. It would be as placing "Geiger counters" at the infinite and horizon boundaries, such that the total number of degrees of freedom is conserved.

Being anchored to the boundary, such boundary degrees of freedom would be nonpropagating ones (in the bulk). In order to grasp their nature and structure, let us consider the expression of the flux in equation 97 , integrating it in the boundary cuts

$$
F(\tau)=\int_{\mathcal{S}_{\mathcal{H}}} \gamma\left|\partial_{\tau} \phi\right|^{2} d S+\int_{\mathcal{S}^{\infty}} \gamma\left|\partial_{\tau} \phi\right|^{2} d S
$$

This is the relevant equation in our line of thought towards the missing degrees of freedom. Indeed, the second term in the right-hand-side has the structure of the Bondi-Sachs flux of energy-momentum at null infinity if: i) $\gamma$ is built from the $\ell=0,1$ spherical harmonics and ii) we identify $\partial_{\tau} \phi$ as a news function $\mathcal{N}$ (see e.g. [4, 5]). However, in general, $\gamma$ will be an arbitrary function on $\mathcal{S}_{\tau}^{\infty}$. In this general situation, the vector field $\xi^{a}$ on $\mathscr{I}^{+}$

$$
\xi^{a}=\gamma \ell^{a}, \mathcal{L}_{\ell} \gamma=0
$$

corresponds to a supertranslation in the asymptotic BMS group (if $\gamma$ is spanned by the $\ell=0,1$ spherical harmonics, this is then a BMS translation, namely in the Poincaré group). In the spirit of the discussion in [4, 5], the expression (integrated in time) corresponding to (231] in the generic $\gamma$ case would be the "Hamiltonian" $H_{\xi}$ (compare with equation (3.18) in [5])

$$
H_{\xi}=\int_{\mathscr{I}+}\left(\gamma \partial_{\tau} \phi \partial_{\tau} \phi+\gamma \partial_{\tau} \phi+\partial_{\tau} \phi \Delta_{\mathcal{S} \infty} \gamma\right) d \tau d S
$$


corresponding to the BMS supertranslation $\xi^{a}$ and generating symplectomorphisms in the (appropriate) phase space of degrees of freedom that "live on the boundary".

The important point is that such expression couples the (missing) degrees of freedom $\phi$, propagating through the bulk and flowing away through the asymptotic boundary, with the BMS elements (namely supertranslations $\xi^{a}$ ) living on the boundary. Moreover, such $\xi^{a}$ supertranslations appear explicitly in the term accounting for the lack of selfadjointness, namely in equation (228), that is the signature of missing degrees of freedom. In the same heuristic spirit, an analogous discussion could be developed at the event horizon (more generally, isolated horizon), in terms of the $\mathbb{R}^{+}-$MOTS-gauge symmetry described in [56, 55] in the MOTS-stability setting, and precisely presented in terms of such $\gamma \ell^{a}$ vectors. In this perspective, $L_{1}$ is a bulk operator accounting for the energy and therefore the scalar product, whereas the operator $L_{2}$ is essentially a BMS object living on the null boundaries.

In this setting, our proposal is that missing degrees of freedom can be encoded in BMS supertranslation degrees of freedom:

i) BMS supertranslations $\xi^{a}$ would stand as a dynamical symmetry, acting on (and generating) the phase space of degrees of freedom on the boundary (the "clicks" in the Geiger counter analogy), with $\gamma$ a degree of freedom living on the null boundaries.

ii) The whole geometric construction starts from the choice of slicing of null boundaries. This is arbitrary, but all choices are related by an appropriate supertranslation.

iii) The set of degrees of freedom $(\phi, \gamma)$ would be complete. By this we mean that, as $\phi$ flows away, degrees of freedom $\gamma$ are "activated" through a coupling controlled by the Hamiltonian 233, so a total energy of the type $E_{o}=E(\phi, \psi)+H_{\xi}$ would be conserved.

Endowing this heuristic (and, admittedly, bold) picture with a sound foundation will be the subject of dedicated research [37]. The hope would be that, from the perspective of the extended space of degrees of freedom $(\phi, \gamma)$, one could gain insight into the observed instability of BH QNMs. Paraphrasing this in terms of inverse scattering, the additional BMS degrees of freedom could provide necessary additional data at the boundaries (complementary to transmission and reflection amplitudes), needed to determine the scattering potential 团

On the other hand, if a conservative formulation of the dynamics can be constructed in the extended space of degrees of freedom $(\phi, \gamma)$, then a notion of global spacetime normal modes, as eigenfunctions of the time evolution generator, could be envisaged. This could be of interest both from a phenomenological and from a fundamental (quantization) perspective.

\section{Conclusions and perspectives}

\subsection{Conclusions}

In this work we have pushed forward the research program on BH QNM instability proposed in [53], with a focus on the role of the scalar product in the pseudospectrum analysis and its specific structural aspects in the hyperboloidal approach to QNMs.

We have obtained the following main results:

i) Derivation of the energy scalar product in the hyperboloidal scheme from a spacetime perspective, recovering the effective expression in [53] and extending it to incorporate more general boundary/asymptotic conditions. Likewise, an explicit expression

$\sharp$ This approach in the spirit of "inverse scattering" theory, connects with the cross-correlation approach to strongfield spacetime dynamics in [52 51, 50] (see also [87, 45]). This also resonates with recent approaches in celestial holography studying bulk dynamics in terms of asymptotic (holographic) data (see e.g. [89] and references therein). 
for the energy flux across spacetime null boundaries has been derived, permitting the identification of the specific element in the formalism accounting for loss of selfadjointness, namely a function $\gamma$ living on null infinity and the horizon sections.

ii) Development of a weak formulation of the BH QNM problem, specially well suited to address low regularity issues in this problem. As an application, demonstration of the genericity of the $\mathrm{BH}$ QNM overtone instability as regards the employed numerical scheme. This is obtained by implementing a finite elements scheme that recovers results from the Chebyshev spectral approach. This addresses one of the caveats in [53], regarding possible artifacts related to Chebyshev's derivation matrices.

iii) Derivation of QNM resonant expansions of the scattered field (e.g. the GW waveform) based on QNM normalizability in the hyperboloidal scheme and Keldysh's asymptotic expansions of the resolvent in a Hilbert (more generally, Banach) space. This recovers Lax-Phillips resonant expansions, adapted to the case of normalizable eigenfunctions and therefore permitting the identification of expansion coefficients. In particular, we provide explicit expressions for the expansion coefficients obtained through projection on left-eigenvectors, valid in spite of the generic loss of orthogonality of eigenfunctions, and reducing to the known expressions for normal modes in the selfadjoint case.

iv) More heuristically, from estimations of the norm of the operator $L$ (the generator of the dynamics) in terms of the energy density of spacetime perturbations, we find evidence that spiked (low-regularity) distributions of $\mathrm{BH}$ perturbations are more efficient in triggering BH QNM instabilities than smooth distributions. On the one hand, this can be of astrophysical interest, suggesting sharp energy/matter concentrations as the preferred candidates among $\mathrm{BH}$ environmental disturbances to impact on $\mathrm{BH}$ spectroscopy through QNM instabilities. For instance, distributions of compact objects around a supermassive BH would impact BH QNMs more than smooth gas distributions. On the other hand, at a fundamental physics level, an effective low-regularity of spacetime (e.g. granularity, stochasticity) emerging from (quantum) first principles, could enhance QNM instability.

Together with these results we have made the following proposals/conjectures:

a) A notion of $\epsilon$-dual QNM expansions for $\mathrm{BH}$ spectroscopy has been introduced, providing equally valid expressions for a GW waveform, up to errors of order $\epsilon$. Specifically, one of the expansions is built on non-perturbed BH QNMs, whereas the other is constructed on "Nollert-Price" QNMs under perturbations of order $\epsilon$. Both expansions are meant to be applied to the same observed waveform to provide complementary ("dual") information on large average scales (non-perturbed QNMs) and small scales (perturbed QNMs).

b) On the basis of the ongoing work on this research program (namely [53, 54, 26, 1] and the present work), we have proposed a general picture of the high-frequency BH QNM instability phenomenon. This provides an effort towards a self-consistent comprehensive framework to encompass the ensemble of different related results found in the literature.

c) As a specific aspect of the latter, intimately related to the concentration mechanism underlying the singularized "spiked-perturbations" for BH QNM instability, we have refined the conjecture proposed in [54] on the limit of QNMs under high-frequency perturbations: in the limit of infinite high-frequency, the asymptotics of Nollert-Price BH QNMs branches converge universally to Regge QNM branches. In such ultraviolet limit, BH QNMs would migrate to asymptotically logarithmic pseudospectra contours, distributing along them in the precise pattern determined by Regge broad resonances. This supports the further conjecture suggesting the universality of (curvature) QNMs of all compact objects, either high-frequency perturbed BHs or matter compact stars. 
7.1.1. Complementary developments in the QNM instability problem. Together with the results and proposals above, the present work provides some additional detailed discussions with either pedagogical or perspective-enlarging value.

In particular, we have illustrated the effect of the choice of the scalar product in eigenvalue instability, by using an elliptic one-dimensional second-order differential operator with constant coefficients. This operator provides a rich illustration of the different subtle issues concerning spectral instability. If use is made of the standard $L^{2}$ scalar product, the operator is non-selfadjoint but it is still "formally normal". However, spectral stability is not realized since the operator is actually non-normal, but this can only be understood at the level of the domains of the operator and its adjoint. We conclude that, if using the associated $L^{2}$-norm, the operator is spectrally unstable, as confirmed by its pseudospectrum. However, using an alternative scalar product determined by the Sturm-Liouville form of the operator, the latter is not only normal but actually selfadjoint. In the associated "Sturm-Liouville" norm the operator is spectrally stable, again confirmed by the calculation of the pseudospectrum. This example illustrates the care required to conclude either spectral stability or instability.

Finally, some elements towards a genuine geometric setting for QNMs in a compactified hyperboloidal approach have been discussed. In particular, the identification of the $L_{2}$ blockoperator as an object essentially depending on the geometry at the conformal null boundaries, and oblivious to the bulk, hints to the plausible role of BMS-supertranslations as the objects controlling non-selfadjointness of the evolution generator $L$ and, therefore, accounting for non-trivial BH pseudospectra and QNM instability. In particular, BMS-charges could be part of the scattering data needed to reconstruct the potential in an inverse scattering approach.

\subsection{Perspectives}

The present work addresses some of the caveats in [53], but many points remain open. In this sense, the present work shares the perspectives in the research program presented in [53]. More specifically related to the present work, we can list the following points:

a) Construction of the energy scalar product for tensorial fields, extending the current scalar product discussion to arbitrary electromagnetic and gravitational $(\mathrm{GW})$ perturbations.

b) Exploration of a scalar product for BH-like (e.g. Pöschl-Teller) potentials rendering a "stable pseudospectrum", with an appropriately weight-modified scalar product. Even if keeping the energy scalar product on the basis of physical considerations, such an alternative scalar product would be informative on the analytical aspects of the problem.

c) Formalization of the $O(\epsilon)$-equivalence of $\epsilon$-dual QNM expansions and assessment of their impact on BH spectroscopy, in particular the possible degeneracy of the associated inverse data analysis problem for retrieving QNM overtones from GW waveforms.

d) Proof of the "Nollert-Price to Regge" BH QNM ultraviolet instability conjecture.

e) Development of the geometric hyperboloidal framework for QNMs, in particular assessing the role of BMS-supertranslations and their relation to inverse scattering.

\section{Acknowledgments}

The authors would like to thank Lamis Al Sheikh, Oscar Meneses-Rojas, Rodrigo PanossoMacedo and Johannes Sjöstrand for numerous and continuous discussions. We also thank Abhay Ashtekar, Piotr Bizon, Graham Cox, Oscar Reula and Jeffrey Winicour for raising 
specific points to be developed in this work, as well as Emanuele Berti, Vitor Cardoso and Kyriakos Destounis for the intense and enriching scientific interaction in the late stage of the project. The numerical implementation discussed in Section 4.1 .3 is based on an adaptation of the FEniCs notebooks and related material of the PDE course of Oscar Reula and Manuel Tiglio. We acknowledge support from The European Union's H2020 ERC Consolidator Grant "Matter and Strong-Field Gravity: New Frontiers in Einstein's Theory", Grant Agreement No. MaGRaTh-646597, the PO FEDER-FSE Bourgogne 2014/2020 program and the EIPHI Graduate School (contract ANR-17-EURE-0002) as part of the ISA 2019 project. We also thank the "Investissements d'Avenir" program through project ISITE-BFC (ANR-15-IDEX03), the ANR "Quantum Fields interacting with Geometry" (QFG) project (ANR-20-CE400018-02), and the Spanish FIS2017-86497-C2-1 project (with FEDER contribution).

[1] L. Al Sheikh \& J. L. Jaramillo, A geometric approach to QNMs in optics: application to pseudospectrum and structural stability, In preparation. .

[2] M. Alcubierre, Introduction to $3+1$ numerical Relativity, Oxford University Press, 2008.

[3] M. Ansorg \& R. Panosso Macedo, Spectral decomposition of black-hole perturbations on hyperboloidal slices, Phys. Rev. D93(12), 124016 (2016).

[4] A. Ashtekar \& M. Streubel, Symplectic Geometry of Radiative Modes and Conserved Quantities at Null Infinity, Proc. Roy. Soc. Lond. A 376, 585-607 (1981).

[5] A. Ashtekar, Geometry and Physics of Null Infinity, (9 2014).

[6] V. Baibhav, E. Berti, V. Cardoso, \& G. Khanna, Black hole spectroscopy: Systematic errors and ringdown energy estimates, Physical Review D 97(4) (Feb 2018).

[7] M. Berger, A panoramic view of Riemannian geometry, Springer, Berlin, 2003.

[8] M. V. Berry, Semiclassically weak reflections above analytic and non-analytic potential barriers, Journal of Physics A: Mathematical and General 15(12), 3693-3704 (dec 1982).

[9] M. V. Berry \& K. E. Mount, Semiclassical approximations in wave mechanics, Reports on Progress in Physics 35(1), 315-397 (jan 1972).

[10] E. Berti, V. Cardoso, \& A. O. Starinets, Quasinormal modes of black holes and black branes, Class. Quant. Grav. 26, 163001 (2009).

[11] E. Berti, V. Cardoso, \& C. M. Will, On gravitational-wave spectroscopy of massive black holes with the space interferometer LISA, Phys. Rev. D73, 064030 (2006).

[12] H. R. Beyer, On the completeness of the quasinormal modes of the Poschl-Teller potential, Commun. Math. Phys. 204, 397-423 (1999).

[13] W.-J. Beyn, Y. Latushkin, \& J. Rottmann-Matthes, Finding eigenvalues of holomorphic Fredholm operator pencils using boundary value problems and contour integrals, arXiv e-prints, arXiv:1210.3952 (Oct. 2012).

[14] W.-J. Beyn, An integral method for solving nonlinear eigenvalue problems, Linear Algebra and its Applications 436(10), 3839-3863 (2012), Special Issue dedicated to Heinrich Voss's 65th birthday.

[15] D. Bindel \& M. Zworski, Theory and computation of resonances in 1d scattering.

[16] Y. Boucher \& L. Al Sheikh, Universal coupled-mode theory formulation of quasi-normal modes in a $1 D$ photonic crystal, Optical and Quantum Electronics 52(10) (Oct. 2020).

[17] G. A. Burnett, The high-frequency limit in general relativity, Journal of Mathematical Physics 30(1), 90-96 (Jan. 1989).

[18] M. Cabero, J. Westerweck, C. D. Capano, S. Kumar, A. B. Nielsen, \& B. Krishnan, The next decade of black hole spectroscopy, Phys. Rev. D101(6), 064044 (2020).

[19] E. S. C. Ching, P. T. Leung, A. Maassen van den Brink, W. M. Suen, S. S. Tong, \& K. Young, Quasinormalmode expansion for waves in open systems, Rev. Mod. Phys. 70, 1545-1554 (Oct 1998).

[20] E. S. C. Ching, P. T. Leung, W. M. Suen, \& K. Young, Quasinormal Mode Expansion for Linearized Waves in Gravitational Systems, Phys. Rev. Lett. 74, 4588-4591 (Jun 1995).

[21] Y. Choquet-Bruhat, Construction de solutions radiatives approchées des equations d'Einstein, Communications in Mathematical Physics 12(1), 16-35 (Mar. 1969).

[22] Y. Choquet-Bruhat, Construction de solutions radiatives approchées des equations d'Einstein, Communications in Mathematical Physics 12(1), 16-35 (Mar. 1969).

[23] M. Dafermos \& I. Rodnianski, Lectures on black holes and linear waves, arXiv e-prints, arXiv:0811.0354 (Nov. 2008).

[24] R. G. Daghigh, M. D. Green, \& J. C. Morey, Significance of Black Hole Quasinormal Modes: A Closer Look, Phys. Rev. D 101(10), 104009 (2020).

[25] E. Davies, Linear Operators and their Spectra, Cambridge Studies in Advanced Mathematics, Cambridge University Press, 2007. 
[26] K. Destounis, R. P. Macedo, E. Berti, V. Cardoso, \& J. L. Jaramillo, Pseudospectrum of Reissner-Nordström black holes: quasinormal mode instability and universality, (7 2021).

[27] O. Dreyer, B. J. Kelly, B. Krishnan, L. S. Finn, D. Garrison, \& R. Lopez-Aleman, Black hole spectroscopy: Testing general relativity through gravitational wave observations, Class. Quant. Grav. 21, 787-804 (2004).

[28] T. A. Driscoll \& L. N. Trefethen, Pseudospectra for the wave equation with an absorbing boundary, Journal of Computational and Applied Mathematics 69(1), 125-142 (1996).

[29] S. Dyatlov \& M. Zworski, Mathematical Theory of Scattering Resonances, Graduate Studies in Mathematics, American Mathematical Society, 2019.

[30] M. Embree \& N. Trefethen, (https://www.cs.ox.ac.uk/pseudospectra/index.html.

[31] F. G. Friedlander, Introduction to the theory of distributions / F. G. Friedlander; with additional material by M. Joshi, Cambridge University Press, Cambridge, UK New York, 2e édition. edition, 1998.

[32] H. Friedrich, On the regular and the asymptotic characteristic initial value problem for Einstein's vacuum field equations, Proc. Roy. Soc. Lond. A 375, 169 (1981).

[33] D. Gajic \& C. Warnick, A model problem for quasinormal ringdown on asymptotically flat or extremal black holes, arXiv:1910.08481(2019).

[34] D. Gajic \& C. Warnick, Quasinormal modes in extremal Reissner-Nordström spacetimes, arXiv:1910.08479 (2019).

[35] J. Galkowski \& M. Zworski, Outgoing solutions via Gevrey-2 properties, (2020).

[36] G. Gamow, Zur Quantentheorie des Atomkernes, Zeitschrift fur Physik 51(3-4), 204-212 (Mar. 1928).

[37] E. Gasperin, J. L. Jaramillo, R. P. Macedo, \& J. A. Valiente-Kroon, A geometric approach to scattering resonances: QNM instability from a "pinching $B M S$ " perspective, In preparation .

[38] M. Giesler, M. Isi, M. A. Scheel, \& S. Teukolsky, Black Hole Ringdown: The Importance of Overtones, Phys. Rev. X9(4), 041060 (2019).

[39] W. L. Goodhue, Scattering Theory for Hyperbolic Systems with Coefficients of Gevrey Type, Transactions of the American Mathematical Society 180, 337-346 (1973).

[40] S. Graffi, V. Grecchi, \& G. Jona-Lasinio, Tunnelling instability via perturbation theory, Journal of Physics A: Mathematical and General 17(15), 2935-2944 (oct 1984).

[41] P. Hintz \& A. Vasy, The global non-linear stability of the Kerr-de Sitter family of black holes, ArXiv e-prints (June 2016).

[42] C. Huneau \& J. Luk, Einstein Equations Under Polarized U (1) Symmetry in an Elliptic Gauge, Commun. Math. Phys. 361(3), 873-949 (2018).

[43] C. Huneau \& J. Luk, High-frequency backreaction for the Einstein equations under polarized $\mathbb{U}(1)$ symmetry, Duke Math. J. 167(18), 3315-3402 (2018).

[44] C. Huneau \& J. Luk, Trilinear compensated compactness and Burnett's conjecture in general relativity, (7 2019).

[45] D. A. B. Iozzo et al., Comparing Remnant Properties from Horizon Data and Asymptotic Data in Numerical Relativity, Phys. Rev. D 103(12), 124029 (2021).

[46] R. A. Isaacson, Gravitational Radiation in the Limit of High Frequency. I. The Linear Approximation and Geometrical Optics, Phys. Rev. 166, 1263-1271 (Feb 1968).

[47] R. A. Isaacson, Gravitational Radiation in the Limit of High Frequency. II. Nonlinear Terms and the Effective Stress Tensor, Phys. Rev. 166, 1272-1280 (Feb 1968).

[48] M. Isi, W. M. Farr, M. Giesler, M. A. Scheel, \& S. A. Teukolsky, Testing the Black-Hole Area Law with GW150914, Phys. Rev. Lett. 127(1), 011103 (2021).

[49] M. Isi, M. Giesler, W. M. Farr, M. A. Scheel, \& S. A. Teukolsky, Testing the no-hair theorem with GW150914, Phys. Rev. Lett. 123(11), 111102 (2019).

[50] J. Jaramillo, R. Macedo, P. Moesta, \& L. Rezzolla, Towards a cross-correlation approach to strong-field dynamics in Black Hole spacetimes, AIP Conf.Proc. 1458, 158-173 (2011).

[51] J. L. Jaramillo, R. P. Macedo, P. Moesta, \& L. Rezzolla, Black-hole horizons as probes of black-hole dynamics II: geometrical insights, Phys.Rev. D85, 084031 (2012).

[52] J. L. Jaramillo, R. P. Macedo, P. Moesta, \& L. Rezzolla, Black-hole horizons as probes of black-hole dynamics I: post-merger recoil in head-on collisions, Phys.Rev. D85, 084030 (2012).

[53] J. L. Jaramillo, R. Panosso Macedo, \& L. Al Sheikh, Pseudospectrum and Black Hole Quasinormal Mode Instability, Phys. Rev. X 11(3), 031003 (2021).

[54] J. L. Jaramillo, R. Panosso Macedo, \& L. A. Sheikh, Gravitational wave signatures of black hole quasinormal mode instability, (5 2021).

[55] J. L. Jaramillo, A perspective on Black Hole Horizons from the Quantum Charged Particle, J. Phys. Conf. Ser. 600(1), 012037 (2015).

[56] J. L. Jaramillo, Black hole horizons and quantum charged particles, Class. Quant. Grav. 32(13), 132001 (2015).

[57] G. Jona-Lasinio, F. Martinelli, \& E. Scoppola, New approach to the semiclassical limit of quantum mechanics. 
I. Multiple tunnelings in one dimension, Communications in Mathematical Physics 80(2), $223-254$ (1981).

[58] T. Kato, Perturbation theory for linear operators; 2nd ed., Grundlehren der mathematischen Wissenschaften A Series of Comprehensive Studies in Mathematics, Springer, Berlin, 1976.

[59] M. V. Keldysh, On the characteristic values and characteristic functions of certain classes of non-self-adjoint equations, in Doklady Akad. Nauk SSSR (NS), volume 77, pages 11-14, 1951.

[60] M. V. Keldysh, On the completeness of the eigenfunctions of some classes of non-selfadjoint linear operators, Uspekhi matematicheskikh nauk 26(4), 15-41 (1971).

[61] K. D. Kokkotas \& B. G. Schmidt, Quasinormal modes of stars and black holes, Living Rev. Rel. 2, 2 (1999).

[62] R. A. Konoplya \& A. Zhidenko, Quasinormal modes of black holes: From astrophysics to string theory, Rev. Mod. Phys. 83, 793-836 (2011).

[63] D. Krejcirik, P. Siegl, M. Tater, \& J. Viola, Pseudospectra in non-Hermitian quantum mechanics, J. Math. Phys. 56(10), 103513 (2015).

[64] M. E. L. Trefethen, The Behavior of Nonnormal Matrices and Operators., Princeton University Press, 2005.

[65] P. Lalanne, W. Yan, K. Vynck, C. Sauvan, \& J.-P. Hugonin, Light Interaction with Photonic and Plasmonic Resonances, Laser \& Photonics Review 12(5), 1700113 (May 2018).

[66] P. Lalanne, W. Yan, K. Vynck, C. Sauvan, \& J. Hugonin, Light Interaction with Photonic and Plasmonic Resonances, Laser \& Photonics Reviews 12(5), 1700113 (2018).

[67] P. D. Lax \& R. S. Phillips, Scattering theory, volume 26 of Pure and Applied Mathematics, Academic Press, Boston, second edition edition, 1989.

[68] P. D. Lax \& R. S. Phillips, A logrithmic bound on the location of the poles of the scattering matrix, Archive for Rational Mechanics and Analysis 40, 268-280 (1971).

[69] P. T. Leung, S. Y. Liu, S. S. Tong, \& K. Young, Time-independent perturbation theory for quasinormal modes in leaky optical cavities, Phys. Rev. A 49, 3068-3073 (Apr 1994).

[70] P. T. Leung, S. Y. Liu, \& K. Young, Completeness and orthogonality of quasinormal modes in leaky optical cavities, Phys. Rev. A 49, 3057-3067 (Apr 1994).

[71] H. Liu, W.-L. Qian, Y. Liu, J.-P. Wu, B. Wang, \& R.-H. Yue, On an alternative mechanism for the black hole echoes, 42021.

[72] J. Luk \& I. Rodnianski, Local Propagation of Impulsive GravitationalWaves, Communications on Pure and Applied Mathematics 68(4), 511-624 (2015).

[73] J. Luk \& I. Rodnianski, Nonlinear interaction of impulsive gravitational waves for the vacuum Einstein equations, Cambridge Journal of Mathematics 5(4), 435-570 (2017).

[74] J. Luk \& I. Rodnianski, High-frequency limits and null dust shell solutions in general relativity, (9 2020).

[75] M. A. H. MacCallum \& A. H. Taub, The averaged Lagrangian and high-frequency gravitational waves, Communications in Mathematical Physics 30(2), 153-169 (June 1973).

[76] E. Maggio, L. Buoninfante, A. Mazumdar, \& P. Pani, How does a dark compact object ringdown?, Phys. Rev. D 102(6), 064053 (2020).

[77] A. Martinez, Resonance free domains for non globally analytic potentials, Ann. Henri Poincaré 3(4), 739756 (2002).

[78] R. Mennicken \& M. Möller, Non-Self-Adjoint Boundary Eigenvalue Problems, ISSN, Elsevier Science, 2003.

[79] H.-P. Nollert, About the significance of quasinormal modes of black holes, Phys. Rev. D53, 4397-4402 (1996).

[80] H.-P. Nollert, Topical Review: Quasinormal modes: the characteristic 'sound' of black holes and neutron stars, Class. Quant. Grav. 16, R159-R216 (1999).

[81] H.-P. Nollert \& R. H. Price, Quantifying excitations of quasinormal mode systems, J. Math. Phys. 40, 9801010 (1999).

[82] I. Ota \& C. Chirenti, Overtones or higher harmonics? Prospects for testing the no-hair theorem with gravitational wave detections, Phys. Rev. D 101(10), 104005 (2020).

[83] I. Ota \& C. Chirenti, Black hole spectroscopy horizons for current and future gravitational wave detectors, 8 2021.

[84] R. Panosso Macedo, Hyperboloidal framework for the Kerr spacetime, Class. Quant. Grav. 37(6), 065019 (2020).

[85] R. Panosso Macedo, J. L. Jaramillo, \& M. Ansorg, Hyperboloidal slicing approach to quasi-normal mode expansions: the Reissner-Nordström case, Phys. Rev. D98(12), 124005 (2018).

[86] D. Pook-Kolb, O. Birnholtz, J. L. Jaramillo, B. Krishnan, \& E. Schnetter, Horizons in a binary black hole merger II: Fluxes, multipole moments and stability, (6 2020).

[87] V. Prasad, A. Gupta, S. Bose, B. Krishnan, \& E. Schnetter, News from horizons in binary black hole mergers, Phys. Rev. Lett. 125(12), 121101 (2020).

[88] W.-L. Qian, K. Lin, C.-Y. Shao, B. Wang, \& R.-H. Yue, On asymptotical quasinormal mode spectrum for piecewise approximate effective potential, arXiv:2009.11627 (9 2020).

[89] A.-M. Raclariu, Lectures on Celestial Holography, 2021. 
[90] T. Regge, Analytic properties of the scattering matrix, Nuovo Cimento (Italy) Divided into Nuovo Cimento A and Nuovo Cimento B Vol: (10) 8 (6 1958).

[91] C. Sauvan, J.-P. Hugonin, I. Maksymov, \& P. Lalanne, Theory of the spontaneous optical emission of nanosize photonic and plasmon resonators, Physical Review Letters 110(23), 237401 (2013).

[92] P. J. Schmid, Nonmodal Stability Theory, Annual Review of Fluid Mechanics 39(1), 129-162 (2007).

[93] B. Simon, Semiclassical analysis of low lying eigenvalues. IV. The flea on the elephant, Journal of Functional Analysis 63, 123-136 (1985).

[94] S. Sivakumar, Quantum Mechanics: Concepts and Applications, 2nd edn., by Nouredine Zettili. Scope: textbook. Level: undergraduates and advanced students, volume 52, July 2011.

[95] J. Sjöstrand, Geometric bounds on the density of resonances for semiclassical problems, Duke Mathematical Journal 60, 1-57 (1990).

[96] J. Sjöstrand, Non-Self-Adjoint Differential Operators, Spectral Asymptotics and Random Perturbations, Birkhäuser Basel, 2019.

[97] J. Sjöstrand, Non-Self-Adjoint Differential Operators, Spectral Asymptotics and Random Perturbations, Pseudo-Differential Operators, Springer International Publishing, 2019.

[98] J. Sjöstrand \& M. Zworski, Asymptotic distribution of resonances for convex obstacles, Acta Mathematica 183(2), $191-253$ (1999).

[99] J. Sjöstrand \& M. Zworski, Fractal upper bounds on the density of semiclassical resonances, Duke Mathematical Journal 137(3), 381 - 459 (2007).

[100] A. Stefanos, Lecture Notes on General Relativity Columbia University, https://web.math.princeton.edu/ aretakis/columbiaGR.pdf (2013).

[101] S.-H. Tang \& M. Zworski, Resonance expansions of scattered waves, Communications on Pure and Applied Mathematics 53(10), 1305-1334 (2000).

[102] L. N. Trefethen, Spectral Methods in MatLab, Society for Industrial and Applied Mathematics, USA, 2000.

[103] L. N. Trefethen, A. E. Trefethen, S. C. Reddy, \& T. A. Driscoll, Hydrodynamic Stability Without Eigenvalues, Science 261(5121), 578-584 (1993).

[104] L. N. Trefethen, A. E. Trefethen, S. C. Reddy, \& T. A. Driscoll, Hydrodynamic Stability Without Eigenvalues, Science 261(5121), 578-584 (1993).

[105] L. Trefethen \& M. Embree, Spectra and Pseudospectra: The Behavior of Nonnormal Matrices and Operators, Princeton University Press, 2005.

[106] A. Vañó Viñuales, S. Husa, \& D. Hilditch, Spherical symmetry as a test case for unconstrained hyperboloidal evolution, Class. Quant. Grav. 32(17), 175010 (2015).

[107] B. R. Vainberg, Exterior elliptic problems that depend polynomially on the spectral parameter, and the asymptotic behavior for large values of the time of the solutions of nonstationary problems, Mat. Sb. (N.S.) 92(134), 224-241 (1973).

[108] J. A. Valiente Kroon, Conformal methods in General Relativity, Cambridge University Press, 2016.

[109] R. M. Wald, General Relativity, Chicago University Press, 1984.

[110] C. M. Warnick, On quasinormal modes of asymptotically anti-de Sitter black holes, Commun. Math. Phys. 333(2), 959-1035 (2015).

[111] F. Zhang, The Schur Complement and its Applications, volume 4 of Numerical Methods and Algorithms, Springer, New York, 2005.

[112] Y. J. Zhang, J. Wu, \& P. T. Leung, High-frequency behavior of w-mode pulsations of compact stars, Phys. Rev. D 83, 064012 (Mar 2011).

[113] M. Zworski, Distribution of poles for scattering on the real line, Journal of Functional Analysis 73(2), 277 296 (1987).

[114] M. Zworski, Resonances in physics and geometry, Notices Amer. Math. Soc. 46, 319-328 (1999).

[115] M. Zworski, Mathematical study of scattering resonances, Bulletin of Mathematical Sciences 7(1), 1-85 (2017). 